Supporting Information for:

\title{
Remarkable Salt Effect on In-Mediated Allylation of \\ $N$-tert-Butanesulfinyl Imines in Aqueous Media: Highly \\ Practical Asymmetric Synthesis of Chiral Homoallylic Amines \\ and Isoindolinones
}

\begin{abstract}
Xing-Wen Sun, Min Liu, Ming-Hua Xu, and Guo-Qiang Lin
Shanghai Institute of Organic Chemistry, Chinese Academy of Sciences, 354 Fenglin Road, Shanghai 200032, and Shanghai Institute of Materia Medica, Chinese Academy of Sciences, 555 Zuchongzhi Road, Shanghai 201203, China.

xumh@mail.sioc.ac.cn
\end{abstract}

\section{Table of Contents}

1. General procedure for the allylation of chiral $N$-tert-butanesulfinyl aldimines in saturated aqueous $\mathrm{NaBr}$ solution.

2. Characterization data of the obtained enantioenriched homoallylic amines.

S2-S9

3. General procedure for the synthesis of chiral 3-allylsubstituted isoindolinones.

4. Characterization data of the obtained chiral 3-allylsubstituted isoindolinones.

S9-S12

5. The synthesis and HPLC of D-allylglycine

$\mathrm{S} 12-\mathrm{S} 13$

6. Copies of ${ }^{1} \mathrm{H}$ and ${ }^{13} \mathrm{C}$ NMR spectra of compounds 2,4 and 5.

S14-S95 
1. General procedure for the allylation of chiral $N$-tert-butanesulfinyl aldimines in saturated aqueous $\mathrm{NaBr}$ solution.

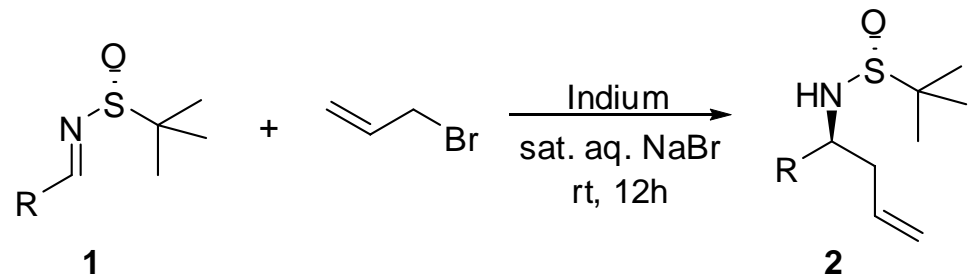

To a suspension of Indium powder (115 mg, $1 \mathrm{mmol})$ and $(R)$-N-tert-butanesulfinyl imines $(0.25 \mathrm{mmol})$ in saturated aqueous $\mathrm{NaBr}$ solution $(5 \mathrm{~mL})$ was added allyl bromide $(1 \mathrm{mmol})$ at room temperature. The resultant mixture was then stirred at $\mathrm{rt}$ for 12 hours. The reaction was quenched with $10 \sim 20 \mathrm{~mL}$ of saturated aqueous $\mathrm{NaHCO}_{3}$. Extraction with ethyl acetate, dried over anhydrous $\mathrm{Na}_{2} \mathrm{SO}_{4}$. After concentrated, the residue was purified by flash column chromatography to afford the desired allylation product.

\section{Characterization data of the obtained enantioenriched homoallylic amines.}<smiles>C=CCC(NS(=O)C(C)(C)C)c1ccccc1</smiles>

$2 a$

$[\alpha]_{\mathrm{D}}{ }^{26}-112.4\left(\right.$ c 1.05, $\left.\mathrm{CHCl}_{3}\right)$ for $94 \% \mathrm{de} ;{ }^{1} \mathrm{H} \mathrm{NMR}(300 \mathrm{MHz}$, $\left.\mathrm{CDCl}_{3}\right): \delta 1.19(\mathrm{~s}, 9 \mathrm{H}), 2.42-2.64(\mathrm{~m}, 2 \mathrm{H}), 3.69(\mathrm{~s}, 1 \mathrm{H}), 4.44-4.50$ $(\mathrm{m}, 1 \mathrm{H}), 5.15-5.21(\mathrm{~m}, 2 \mathrm{H})$, 5.66-5.80 (m, 1H), 7.24-7.37 (m, 5H) ppm; ${ }^{13} \mathrm{C}$ NMR $\left(100 \mathrm{MHz}, \mathrm{CDCl}_{3}\right) \delta 22.51,43.30,55.53,57.06$, 119.14, 127.40, 127.55, 128.36, 134.07, 141.64 ppm; FT-IR (film, $\left.\mathrm{cm}^{-1}\right) \vee 3033,2927,2869,2235,1640,1456,1058,911,734,701$;

ESI-MS: $252.2\left(\mathrm{M}^{+}+\mathrm{H}\right), 274.2\left(\mathrm{M}^{+}+\mathrm{Na}\right)$; HRMS (EI) for $\mathrm{C}_{14} \mathrm{H}_{21} \mathrm{NOS}$ : calcd. 251.1344 , found 251.1348 .<smiles>C=CCC(NS(=O)C(C)(C)C)c1ccc(Cl)cc1</smiles>

$[\alpha]_{\mathrm{D}}{ }^{26}-128.7\left(\mathrm{c} 1.00, \mathrm{CHCl}_{3}\right)$ for $95 \% \mathrm{de} ;{ }^{1} \mathrm{H} \mathrm{NMR}(300 \mathrm{MHz}$, $\left.\mathrm{CDCl}_{3}\right): \delta 1.19(\mathrm{~s}, 9 \mathrm{H}), 2.39-2.61(\mathrm{~m}, 2 \mathrm{H}), 3.70(\mathrm{~s}, 1 \mathrm{H})$, 4.42-4.74 (m, 1H), 5.15-5.20 (m, 2H), 5.64-5.78 (m, $1 \mathrm{H})$, 7.24-7.33 (m, 5H) ppm; ${ }^{13} \mathrm{C} \mathrm{NMR}\left(100 \mathrm{MHz}, \mathrm{CDCl}_{3}\right) \delta 22.52$, 43.22, 55.64, 55.41, 119.51, 128.63, 128.84, 133.35, 133.69, 140.20 ppm; FT-IR $\left(\mathrm{KBr}, \mathrm{cm}^{-1}\right) \vee 3201,3079,2981,2956$, 2867, 1489, 1408, 1900, 827, 647; ESI-MS (m/z, \%): $286.2\left(\mathrm{M}^{+}+\mathrm{H}\right)$; HRMS (EI) for $\mathrm{C}_{14} \mathrm{H}_{20}$ CINOS: calcd. 285.0954, found 285.0949.<smiles>C=CCC(NS(=O)C(C)(C)C)c1ccc(C)cc1</smiles>

2c

$[\alpha]_{\mathrm{D}}{ }^{26}-136.7\left(\mathrm{c} 1.20, \mathrm{CHCl}_{3}\right)$ for $96 \%$ ee; ${ }^{1} \mathrm{H}$ NMR $(300 \mathrm{MHz}$, $\left.\mathrm{CDCl}_{3}\right): \delta 1.19(\mathrm{~s}, 9 \mathrm{H}), 2.34(\mathrm{~s}, 3 \mathrm{H}), 2.40-2.62(\mathrm{~m}, 2 \mathrm{H}), 3.67(\mathrm{~s}$, $1 \mathrm{H}), 4.41-4.46(\mathrm{~m}, 1 \mathrm{H}), 5.14-5.20(\mathrm{~m}, 2 \mathrm{H}), 5.66-5.80(\mathrm{~m}, 1 \mathrm{H})$, $7.18(\mathrm{dd}, 2 \mathrm{H}, \mathrm{J}=8.4,20.7 \mathrm{~Hz}) \mathrm{ppm} ;{ }^{13} \mathrm{C} \mathrm{NMR}(100 \mathrm{MHz}$, $\left.\mathrm{CDCl}_{3}\right) \delta 21.09,22.56,43.41,55.54,56.81,119.07,127.37$, 129.13, 134.28, 137.26, 138.62 ppm; FT-IR (film, $\mathrm{cm}^{-1}$ ) $v$ 3204, 2976, 2863, 1643, 1053, 816 ; ESI-MS (m/z, \%): $266.2\left(\mathrm{M}^{+}+\mathrm{H}\right), 288.2\left(\mathrm{M}^{+}+\mathrm{Na}\right)$; 
HRMS (EI) for $\mathrm{C}_{15} \mathrm{H}_{23} \mathrm{NOS}$ : calcd. 265.1500, found 265.1512.<smiles>C=CC[C@@H](N[S@](=O)C(C)(C)C)c1ccc(C(F)(F)F)cc1</smiles>

2d

$[\alpha]_{\mathrm{D}}^{26}-82.1\left(\mathrm{c} 1.00, \mathrm{CHCl}_{3}\right)$ for $94 \%$ ee; ${ }^{1} \mathrm{H}$ NMR $(500 \mathrm{MHz}$, $\left.\mathrm{CDCl}_{3}\right): \delta 1.21(\mathrm{~s}, 9 \mathrm{H}), 2.45-2.51(\mathrm{~m}, 1 \mathrm{H}), 2.58-2.63(\mathrm{~m}, 1 \mathrm{H})$, $3.74(\mathrm{~s}, 1 \mathrm{H}), 4.54-4.56(\mathrm{~m}, 1 \mathrm{H}), 5.18-5.21(\mathrm{~m}, 2 \mathrm{H}), 5.67-5.74$ $(\mathrm{m}, 1 \mathrm{H}), 7.45(\mathrm{~d}, 2 \mathrm{H}, \mathrm{J}=8.03 \mathrm{~Hz}), 7.60(\mathrm{~d}, 2 \mathrm{H}, \mathrm{J}=8.08 \mathrm{~Hz})$ ppm; ${ }^{13} \mathrm{C}$ NMR (125 MHz, $\left.\mathrm{CDCl}_{3}\right) \delta 22.48,43.13,55.75$, $56.61,119.76,122.98,125.14,125.39,125.42,127.79,129.73$, 129.98, 133.41, 145.86 ppm; FT-IR (film, $\mathrm{cm}^{-1}$ ) v 3208, 2975, 2859, 1650, 1049, 809 ; ESI-MS: $320.1\left(\mathrm{M}^{+}+\mathrm{H}\right)$; HRMS (ESI) for $\left[\mathrm{C}_{15} \mathrm{H}_{20} \mathrm{~F}_{3} \mathrm{NNaOS}\right]^{+}$: calcd. 342.1115 , found 342.1119 .<smiles>C=CC[C@@H](N[S@](=O)C(C)(C)C)c1ccc(OC)cc1</smiles>

$[\alpha]_{\mathrm{D}}^{26}-86.9\left(\mathrm{c} 1.05, \mathrm{CHCl}_{3}\right)$ for $96 \%$ de; ${ }^{1} \mathrm{H}$ NMR $(300 \mathrm{MHz}$, $\left.\mathrm{CDCl}_{3}\right): \delta 1.19(\mathrm{~s}, 9 \mathrm{H}), 2.39-2.61(\mathrm{~m}, 2 \mathrm{H}), 3.67(\mathrm{~s}, 1 \mathrm{H})$, $3.80(\mathrm{~s}, 3 \mathrm{H}), 4.39-4.44(\mathrm{~m}, 1 \mathrm{H}), 5.14-5.20(\mathrm{~m}, 2 \mathrm{H}), 5.66-5.80$ (m, 1H), $6.87(\mathrm{~d}, 2 \mathrm{H}, \mathrm{J}=8.4 \mathrm{~Hz}), 7.24(\mathrm{~d}, 2 \mathrm{H}, \mathrm{J}=8.4 \mathrm{~Hz})$ ppm; ${ }^{13} \mathrm{C}$ NMR $\left(100 \mathrm{MHz}, \mathrm{CDCl}_{3}\right) \delta 22.63,43.48,55.22$, $55.54,56.48,113.84,119.11,128.66,133.62,134.36,159.06$ ppm; FT-IR $\left(\mathrm{KBr}, \mathrm{cm}^{-1}\right)$ v3450, 3283, 3216, 3076, 2958, 2869, 1612, 1514, 7247, 1056, 832; ESI-MS (m/z, \%):282.2 $\left(\mathrm{M}^{+}+\mathrm{H}\right), 304.2\left(\mathrm{M}^{+}+\mathrm{Na}\right)$; HRMS (EI) for $\mathrm{C}_{15} \mathrm{H}_{23} \mathrm{NO}_{2} \mathrm{~S}$ : calcd. 281.1449, found 281.1452.

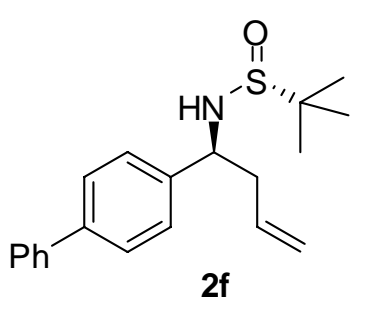

$[\alpha]_{\mathrm{D}}{ }^{26}-70.1\left(\mathrm{c} 0.95, \mathrm{CHCl}_{3}\right)$ for $98 \% \mathrm{de} ;{ }^{1} \mathrm{H}$ NMR $(300 \mathrm{MHz}$, $\left.\mathrm{CDCl}_{3}\right): \delta 1.21(\mathrm{~s}, 9 \mathrm{H}), 2.48-2.66(\mathrm{~m}, 2 \mathrm{H}), 3.74(\mathrm{~s}, 1 \mathrm{H})$, 4.52-4.54 (m, $1 \mathrm{H}), 5.18-5.24(\mathrm{~m}, 2 \mathrm{H}), 5.73-5.83(\mathrm{~m}, 1 \mathrm{H})$, 7.33-7.60 (m, 9H) ppm; ${ }^{13} \mathrm{C}$ NMR $\left(75 \mathrm{MHz}, \mathrm{CDCl}_{3}\right) \delta 20.75$, $41.59,53.81,54.80,117.56,125.18,125.30,125.43,126.03$, $126.89,132.30,138.61,138.80,138.88$ ppm; FT-IR (film, $\mathrm{cm}^{-1}$ ) $v$ 3208, 2980, 2855, 1645, 1060, 766 ; ESI-MS: $328.2\left(\mathrm{M}^{+}+\mathrm{H}\right)$; HRMS (ESI) for $\left[\mathrm{C}_{20} \mathrm{H}_{25} \mathrm{NNaOS}\right]^{+}$: calcd. 350.1555 , found 350.1557 .<smiles>C=CCC(NS(=O)C(C)(C)C)c1cccc(Cl)c1</smiles>

$[\alpha]_{\mathrm{D}}{ }^{26}-83.1\left(\mathrm{c} 0.90, \mathrm{CHCl}_{3}\right)$ for $98 \% \mathrm{de} ;{ }^{1} \mathrm{H}$ NMR $(500 \mathrm{MHz}$, $\left.\mathrm{CDCl}_{3}\right): \delta 1.19(\mathrm{~s}, 9 \mathrm{H}), 2.44-2.56(\mathrm{~m}, 1 \mathrm{H}), 2.57-2.60(\mathrm{~m}, 1 \mathrm{H})$, $3.70(\mathrm{~s}, 1 \mathrm{H}), 4.43-4.46(\mathrm{~m}, 1 \mathrm{H}), 5.17-5.20(\mathrm{~m}, 2 \mathrm{H}), 5.69-5.72$ $(\mathrm{m}, 1 \mathrm{H}), 7.19-7.32(\mathrm{~m}, 4 \mathrm{H}) \mathrm{ppm} ;{ }^{13} \mathrm{C} \mathrm{NMR}\left(125 \mathrm{MHz}, \mathrm{CDCl}_{3}\right)$ $\delta 22.49,43.14,55.69,56.54,119.59,125.68,127.51,127.74$, $2 \mathrm{~g}$ 129.68, 133.56, 134.30, 143.87 ppm; FT-IR (film, $\mathrm{cm}^{-1}$ ) v 3201, 2980, 2871, 1651, 1048; ESI-MS: $286.1 \quad\left(\mathrm{M}^{+}+\mathrm{H}\right)$; HRMS (ESI) for $\left[\mathrm{C}_{14} \mathrm{H}_{20} \mathrm{ClNaNOS}\right]^{+}$: calcd. 308.0852, found 308.0850.<smiles>C=CCC(NS(=O)C(C)(C)C)c1cccc(OC)c1</smiles>

$2 \mathrm{~h}$ $[\alpha]_{\mathrm{D}}{ }^{26}-56.2\left(\mathrm{c} 1.04, \mathrm{CHCl}_{3}\right)$ for $97 \% \mathrm{de} ;{ }^{1} \mathrm{H}$ NMR $(400 \mathrm{MHz}$, $\left.\mathrm{CDCl}_{3}\right): \delta 1.19(\mathrm{~s}, 9 \mathrm{H}), 2.44-2.50(\mathrm{~m}, 1 \mathrm{H}), 2.55-2.60(\mathrm{~m}, 1 \mathrm{H})$, $3.68(\mathrm{~s}, 1 \mathrm{H}), 3.80(\mathrm{~s}, 3 \mathrm{H}), 4.43-4.46(\mathrm{~m}, 1 \mathrm{H}), 5.16-5.20(\mathrm{~m}$, $2 \mathrm{H}), 5.70-5.74(\mathrm{~m}, 1 \mathrm{H}), 6.80-6.92(\mathrm{~m}, 3 \mathrm{H}), 7.23-7.26(\mathrm{~m}, 1 \mathrm{H})$ 
ppm; ${ }^{13} \mathrm{C}$ NMR $\left(125 \mathrm{MHz}, \mathrm{CDCl}_{3}\right) \delta 22.53,43.29,55.09,55.59,56.99,112.94$, 113.00, 119.18, 119.76, 129.39, 134.07, 143.38, 159.64 ppm; FT-IR (film, cm-1) $v$ 3199, 2980, 2871, 1643, 820 ; ESI-MS: $282.1\left(\mathrm{M}^{+}+\mathrm{H}\right)$; HRMS (ESI) for $\left[\mathrm{C}_{15} \mathrm{H}_{23} \mathrm{NNaO}_{2} \mathrm{~S}\right]^{+}$: calcd. 304.1347, found 304.1345.

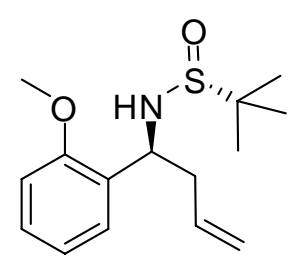

$2 \mathbf{i}$

$[\alpha]_{\mathrm{D}}{ }^{26}-75.2\left(\mathrm{c} 1.03, \mathrm{CHCl}_{3}\right)$ for $99 \%$ de; ${ }^{1} \mathrm{H} \mathrm{NMR}(500 \mathrm{MHz}$, $\left.\mathrm{CDCl}_{3}\right): \delta 1.18(\mathrm{~s}, 9 \mathrm{H}), 2.50-2.56(\mathrm{~m}, 1 \mathrm{H}), 2.64-2.69(\mathrm{~m}, 1 \mathrm{H}), 3.82$ $(\mathrm{s}, 1 \mathrm{H}), 3.84(\mathrm{~s}, 3 \mathrm{H}), 4.83-4.87(\mathrm{~m}, 1 \mathrm{H}), 5.10-5.15(\mathrm{~m}, 2 \mathrm{H})$, 5.69-5.77 (m, 1H), 6.87-6.94 (m, 2H), 7.22-7.26 (m, 2H) ppm; ${ }^{13} \mathrm{C}$ NMR $\left(125 \mathrm{MHz}, \mathrm{CDCl}_{3}\right) \delta 22.53,41.51,52.63,55.31,55.63$, $110.63,118.43,120.32,127.78,128.22,129.98,134.69,156.80$ ppm; FT-IR (film, $\mathrm{cm}^{-1}$ ) v 3201, 2980, 2859, 1644, 1050, 811 ; ESI-MS: 282.1 $\left(\mathrm{M}^{+}+\mathrm{H}\right)$; HRMS (ESI) for $\left[\mathrm{C}_{15} \mathrm{H}_{23} \mathrm{NNaO}_{2} \mathrm{~S}\right]^{+}$: calcd. 304.1347, found 304.1341.

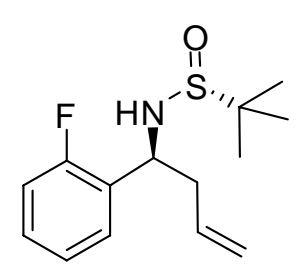

2j

$[\alpha]_{\mathrm{D}}{ }^{26}-85.9\left(\mathrm{c} 1.00, \mathrm{CHCl}_{3}\right)$ for $99 \%$ de; ${ }^{1} \mathrm{H} \mathrm{NMR}(300 \mathrm{MHz}$, $\left.\mathrm{CDCl}_{3}\right): \delta 1.20(\mathrm{~s}, 9 \mathrm{H}), 2.51-2.71(\mathrm{~m}, 2 \mathrm{H}), 3.70(\mathrm{~d}, 1 \mathrm{H}, \mathrm{J}=2.4 \mathrm{~Hz})$, 4.77-4.83 (m, 1H), 5.14-5.20 (m, 2H), 5.66-5.80 (m, 1H), 7.01-7.36 (m, 4H) ppm; ${ }^{9} \mathrm{~F}$ NMR: $\delta 55.43(\mathrm{~d}, \mathrm{~J}=4.5 \mathrm{~Hz}, 1 \mathrm{~F}) ;{ }^{13} \mathrm{C}$ NMR $(75$ $\left.\mathrm{MHz}, \mathrm{CDCl}_{3}\right) \delta 22.56,41.74,51.96,55.84,115.53,115.82,119.37$, $124.04,124.09,128.71,128.79,128.99,129.10,133.80,158.94$ ppm; FT-IR (film, $\mathrm{cm}^{-1}$ ) $\vee$ 3199, 2971, 2860, 1649, 1058, 820; ESI-MS: 270.1 $\left(\mathrm{M}^{+}+\mathrm{H}\right)$; HRMS (ESI) for $\left[\mathrm{C}_{14} \mathrm{H}_{20} \mathrm{FNNaOS}\right]^{+}$: calcd. 292.1147, found 292.1149.<smiles>C=CC[C@H](NS(=O)C(C)(C)C)c1ccccc1Br</smiles>

2k

$[\alpha]_{\mathrm{D}}{ }^{26}-79.8\left(\mathrm{c} 1.05, \mathrm{CHCl}_{3}\right)$ for $99 \% \mathrm{de} ;{ }^{1} \mathrm{H} \mathrm{NMR}(300 \mathrm{MHz}$, $\left.\mathrm{CDCl}_{3}\right): \delta 1.22(\mathrm{~s}, 9 \mathrm{H}), 2.39-2.49(\mathrm{~m}, 1 \mathrm{H}), 2.66-2.75(\mathrm{~m}, 2 \mathrm{H}), 3.75$ $(\mathrm{d}, 1 \mathrm{H}, \mathrm{J}=1.8 \mathrm{~Hz}), 4.96-5.01(\mathrm{~m}, 1 \mathrm{H}), 5.19-5.24(\mathrm{~m}, 2 \mathrm{H})$, 5.71-5.84 (m, 1H), $7.13(\mathrm{t}, 1 \mathrm{H}, \mathrm{J}=6.9 \mathrm{~Hz}), 7.31(\mathrm{t}, 1 \mathrm{H}, \mathrm{J}=7.2 \mathrm{~Hz})$, $7.41(\mathrm{~d}, 1 \mathrm{H}, \mathrm{J}=7.8 \mathrm{~Hz}), 7.55(\mathrm{~d}, 1 \mathrm{H}, \mathrm{J}=8.4 \mathrm{~Hz}) \mathrm{ppm} ;{ }^{13} \mathrm{C} \mathrm{NMR}$ $\left(75 \mathrm{MHz}, \mathrm{CDCl}_{3}\right) \delta 22.63,41.61,55.89,55.93,119.60,123.47$, 127.43, 128.74, 128.88, 133.10, 133.78, 140.83 ppm; FT-IR (film, $\mathrm{cm}^{-1}$ ) v 3281, 3201, 3076, 2957, 2923, 2867, 1604, 1570, 1056, 996, 916, 784, 696; ESI-MS (m/z, \%): $330.1\left(\mathrm{M}^{+}+\mathrm{H}\right)$; HRMS (EI) for $\mathrm{C}_{14} \mathrm{H}_{20}$ BrNOS: calcd. 329.0449, found 329.0456.<smiles>C=CCC(NS(=O)C(C)(C)C)c1ccc(Cl)cc1Cl</smiles>

$[\alpha]_{\mathrm{D}}{ }^{26}-85.8\left(\mathrm{c} 1.00, \mathrm{CHCl}_{3}\right)$ for $99 \% \mathrm{de} ;{ }^{1} \mathrm{H} \mathrm{NMR}(500 \mathrm{MHz}$, $\left.\mathrm{CDCl}_{3}\right): \delta 1.21(\mathrm{~s}, 9 \mathrm{H}), 2.42-2.48(\mathrm{~m}, 1 \mathrm{H}), 2.63-2.68(\mathrm{~m}, 1 \mathrm{H})$, $3.71(\mathrm{~d}, 1 \mathrm{H}, \mathrm{J}=2.1 \mathrm{~Hz}), 4.95-4.98(\mathrm{~m}, 1 \mathrm{H}), 5.17-5.20(\mathrm{~m}, 2 \mathrm{H})$, 5.69-5.76 (m, 1H), 7.23-7.38 (m, 3H) ppm; ${ }^{13} \mathrm{C}$ NMR $(125$ $\left.\mathrm{MHz}, \mathrm{CDCl}_{3}\right) \delta 22.50,41.23,53.15,55.86,119.73,127.14$, 129.45, 133.32, 133.57, 133.81, 137.90 ppm; FT-IR (film, cm-1) $v$ 3201, 2966, 2868, 1645, 1057, 819 ; ESI-MS (m/z, \%):320.1 $\left(\mathrm{M}^{+}+\mathrm{H}\right)$; HRMS (ESI) for $\left[\mathrm{C}_{14} \mathrm{H}_{19} \mathrm{Cl}_{2} \mathrm{NNaOS}\right]^{+}$: calcd. 342.0462, found 342.0461. 
<smiles>C=CC[C@H](NS(=O)C(C)(C)C)c1ccc(OC)cc1OC</smiles>

$[\alpha]_{\mathrm{D}}{ }^{26}-85.8\left(\mathrm{c} 1.00, \mathrm{CHCl}_{3}\right)$ for $99 \% \mathrm{de},{ }^{1} \mathrm{H} \mathrm{NMR}(300 \mathrm{MHz}$, $\left.\mathrm{CDCl}_{3}\right): \delta 1.18(\mathrm{~s}, 9 \mathrm{H}), 2.44-2.68(\mathrm{~m}, 2 \mathrm{H}), 3.77$ (d-like, $\left.1 \mathrm{H}\right)$, $3.80(\mathrm{~s}, 3 \mathrm{H}), 3.81(\mathrm{~s}, 3 \mathrm{H}), 4.74-4.80(\mathrm{~m}, 1 \mathrm{H}), 5.09-5.16(\mathrm{~m}$, $2 \mathrm{H}), 5.66-5.80(\mathrm{~m}, 1 \mathrm{H}), 6.45-6.48(\mathrm{~m}, 2 \mathrm{H}), 7.16(\mathrm{~d}, 1 \mathrm{H}, \mathrm{J}=$ $9.0 \mathrm{~Hz}) \mathrm{ppm} ;{ }^{13} \mathrm{C} \mathrm{NMR}\left(75 \mathrm{MHz}, \mathrm{CDCl}_{3}\right) \delta 22.63,41.76$, $52.28,55.29,55.42,55.64,98.55,104.13,118.37,122.42$,

128.49, 134.99, 157.98, 160.03 ppm; FT-IR (film, $\mathrm{cm}^{-1}$ ) v 3201, 2971, 2859, 1643, 1055, 814; ESI-MS: $312.2\left(\mathrm{M}^{+}+\mathrm{H}\right)$; HRMS (ESI) for $\left[\mathrm{C}_{16} \mathrm{H}_{25} \mathrm{NNaO}_{3} \mathrm{~S}\right]^{+}$: calcd. 334.1453 , found 334.1451 .<smiles>C=CC[C@H](NS(=O)C(C)(C)C)c1ccc(OC)c(OC)c1</smiles>

2n $[\alpha]_{\mathrm{D}}{ }^{26}-109.8\left(\mathrm{c} 1.15, \mathrm{CHCl}_{3}\right)$ for $97 \% \mathrm{de} ;{ }^{1} \mathrm{H}$ NMR $(300$ $\left.\mathrm{MHz}, \mathrm{CDCl}_{3}\right): \delta 1.21(\mathrm{~s}, 9 \mathrm{H}), 2.39-2.62(\mathrm{~m}, 2 \mathrm{H}), 3.68(\mathrm{~s}, 1 \mathrm{H})$, $3.87(\mathrm{~s}, 3 \mathrm{H}), 3.88(\mathrm{~s}, 3 \mathrm{H}), 4.39-4.44(\mathrm{~m}, 1 \mathrm{H}), 5.16-5.22(\mathrm{~m}$, 2H), 5.68-5.80 (m, 1H), 6.82-6.90 (m, 3H) ppm; ${ }^{13} \mathrm{C}$ NMR $\left(75 \mathrm{MHz}, \mathrm{CDCl}_{3}\right) \delta 22.61,43.52,55.51,55.80,55.84,56.70$, $110.35,110.91,119.14,119.91,134.13,134.31,148.47$, 148.96 ppm; FT-IR (film, cm ${ }^{-1}$ ) v 3203, 2974, 2861, 1640, 1049, 814 ; ESI-MS: 312.2 $\left(\mathrm{M}^{+}+\mathrm{H}\right)$; HRMS (ESI) for $\left[\mathrm{C}_{16} \mathrm{H}_{25} \mathrm{NNaO}_{3} \mathrm{~S}\right]^{+}$: calcd. 334.1453, found 334.1456.

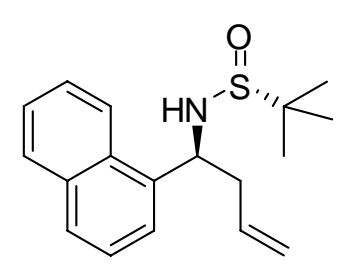

20

$[\alpha]_{\mathrm{D}}{ }^{26}-135.0\left(\mathrm{c} 1.00, \mathrm{CHCl}_{3}\right)$ for $96 \%$ de; ${ }^{1} \mathrm{H} \mathrm{NMR}(300 \mathrm{MHz}$, $\left.\mathrm{CDCl}_{3}\right): \delta 1.21(\mathrm{~s}, 9 \mathrm{H}), 2.61-2.71(\mathrm{~m}, 1 \mathrm{H}), 2.79-2.88(\mathrm{~m}, 1 \mathrm{H})$, $3.85(\mathrm{~d}, 1 \mathrm{H}, \mathrm{J}=1.8 \mathrm{~Hz}), 5.17-5.34(\mathrm{~m}, 3 \mathrm{H}), 5.72-5.86(\mathrm{~m}, 1 \mathrm{H})$, 7.43-7.59 (m, 4H), $7.78(\mathrm{~d}, 1 \mathrm{H}, \mathrm{J}=8.4 \mathrm{~Hz}), 7.87(\mathrm{~d}, 1 \mathrm{H}, \mathrm{J}=8.4$ $\mathrm{Hz}), 8.19(\mathrm{~d}, 1 \mathrm{H}, \mathrm{J}=8.1 \mathrm{~Hz}) \mathrm{ppm} ;{ }^{13} \mathrm{C} \mathrm{NMR}\left(75 \mathrm{MHz}, \mathrm{CDCl}_{3}\right) \delta$ $22.70,42.36,53.57,55.82,119.40,123.18,125.06,125.26$, 125.65, 126.19, 128.20, 129.05, 131.01, 134.03, 134.32, 137.23 ppm; FT-IR (film, $\left.\mathrm{cm}^{-1}\right) \vee 3281,3209,2957,2923,2866,1639,1597,1511,1364,1169,1063,920,779$, 751; ESI-MS (m/z, \%): $302.2\left(\mathrm{M}^{+}+\mathrm{H}\right)$; HRMS (EI) for $\mathrm{C}_{18} \mathrm{H}_{23} \mathrm{NOS}$ : calcd. 301.1500, found 301.1509 .

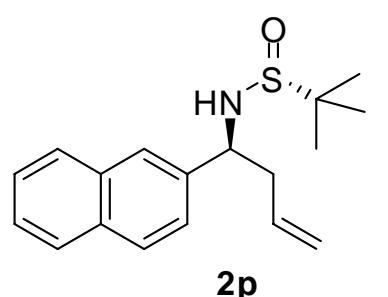

$2 p$

$[\alpha]_{\mathrm{D}}{ }^{26}-111.0\left(\mathrm{c} 1.51, \mathrm{CHCl}_{3}\right)$ for $97 \% \mathrm{de} ;{ }^{1} \mathrm{H} \mathrm{NMR}(300 \mathrm{MHz}$, $\left.\mathrm{CDCl}_{3}\right): \delta 1.19(\mathrm{~s}, 9 \mathrm{H}), 2.51-2.71(\mathrm{~m}, 2 \mathrm{H}), 3.77(\mathrm{~s}, 1 \mathrm{H})$, 4.61-4.66 (m, 1H), 5.15-5.23 (m, 2H), 5.69-5.82 (m, 1H), 7.42-7.50 (m, 3H), 7.78-7.84 (m, 4H) ppm; ${ }^{13} \mathrm{C}$ NMR $(100 \mathrm{MHz}$, $\left.\mathrm{CDCl}_{3}\right) \delta 22.51,43.09,55.53,57.24,119.20,124.97,125.88$, $126.10,126.79,127.59,127.84,128.25,132.95,133.17,134.03$, 138.90 ppm; FT-IR (KBr, cm-1) v 3254, 3057, 2980, 2962, 2924, 2866, 1366, 1053, 925, 827, 756, 650, 598; ESI-MS (m/z, \%): $302.2\left(\mathrm{M}^{+}+\mathrm{H}\right), 324.2\left(\mathrm{M}^{+}+\mathrm{Na}\right)$; HRMS (EI) for $\mathrm{C}_{18} \mathrm{H}_{23} \mathrm{NOS}$ : calcd. 301.1500, found 301.1506.

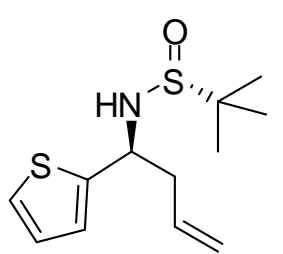

$2 q$

$[\alpha]_{\mathrm{D}}{ }^{26}-63.0\left(\mathrm{c} 1.00, \mathrm{CHCl}_{3}\right)$ for $89 \% \mathrm{de} ;{ }^{1} \mathrm{H} \mathrm{NMR}(300 \mathrm{MHz}$, $\left.\mathrm{CDCl}_{3}\right): \delta 1.22(\mathrm{~s}, 9 \mathrm{H}), 2.55-2.77(\mathrm{~m}, 2 \mathrm{H}), 3.80(\mathrm{~s}, 1 \mathrm{H}), 4.72-4.82$ $(\mathrm{m}, 1 \mathrm{H}), 5.19-5.25(\mathrm{~m}, 2 \mathrm{H}), 5.69-5.83(\mathrm{~m}, 1 \mathrm{H}), 6.94-7.01(\mathrm{~m}, 2 \mathrm{H})$, $7.24(\mathrm{~d}, 1 \mathrm{H}, \mathrm{J}=4.8 \mathrm{~Hz}) \mathrm{ppm} ;{ }^{13} \mathrm{C} \mathrm{NMR}\left(75 \mathrm{MHz}, \mathrm{CDCl}_{3}\right) \delta 22.65$, 
43.75, 53.63, 55.95, 118.64, 119.86, 124.98, 125.18, 126.64, 133.51 ppm; FT-IR (film, $\left.\mathrm{cm}^{-1}\right) \vee 3243,1434,1312,1159,699$, 562.; ESI-MS: $258.1\left(\mathrm{M}^{+}+\mathrm{H}\right)$; HRMS (ESI) for $\left[\mathrm{C}_{12} \mathrm{H}_{19} \mathrm{NNaOS}_{2}\right]^{+}$: calcd. 280.0806, found 280.0804.

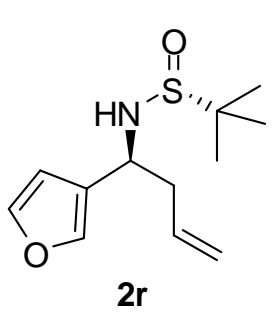

$[\alpha]_{\mathrm{D}}{ }^{26}-35.0\left(\mathrm{c} 0.95, \mathrm{CHCl}_{3}\right)$ for $90 \%$ de; ${ }^{1} \mathrm{H}$ NMR $(300 \mathrm{MHz}$, $\left.\mathrm{CDCl}_{3}\right): \delta 1.20(\mathrm{~s}, 9 \mathrm{H}), 2.45-2.64(\mathrm{~m}, 2 \mathrm{H}), 3.60(\mathrm{~d}, 1 \mathrm{H}, \mathrm{J}=1.8 \mathrm{~Hz})$, 4.43-4.48 (m, 2H), 5.16-5.22 (m, 2H), 5.68-5.82 (m, 1H), $6.34(\mathrm{~s}$, $1 \mathrm{H}), 7.39(\mathrm{~d}, 2 \mathrm{H}, \mathrm{J}=2.1 \mathrm{~Hz}) \mathrm{ppm} ;{ }^{13} \mathrm{C}$ NMR $\left(75 \mathrm{MHz}, \mathrm{CDCl}_{3}\right) \delta$ $22.60,41.99,49.55,55.62,109.02,119.46,126.17,133.79,140.18$, 143.42 ppm; FT-IR (film, $\left.\mathrm{cm}^{-1}\right) \vee 3226,2956,1543,1055,831$; ESI-MS : $242.1\left(\mathrm{M}^{+}+\mathrm{H}\right)$; HRMS (ESI) for $\left[\mathrm{C} 12 \mathrm{H} 19 \mathrm{NNaO}_{2} \mathrm{~S}\right]^{+}$: calcd. 264.1034, found 264.1039 .

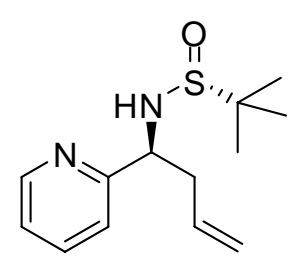

2s

$[\alpha]_{\mathrm{D}}{ }^{26}-69.1\left(\mathrm{c} 0.55, \mathrm{CHCl}_{3}\right)$ for $93 \%$ de; ${ }^{1} \mathrm{H}$ NMR $(300 \mathrm{MHz}$, $\left.\mathrm{CDCl}_{3}\right): \delta 1.26(\mathrm{~s}, 9 \mathrm{H}), 2.58-2.62(\mathrm{~m}, 2 \mathrm{H}), 4.47-4.54(\mathrm{~m}, 1 \mathrm{H}), 4.86$ $(\mathrm{d}, 1 \mathrm{H}, \mathrm{J}=6.6 \mathrm{~Hz}), 5.01-5.06(\mathrm{~m}, 2 \mathrm{H}), 5.64-5.77(\mathrm{~m}, 1 \mathrm{H})$, 7.16-7.29 (m, 2H), $7.65(\mathrm{t}, 1 \mathrm{H}, \mathrm{J}=8.1 \mathrm{~Hz}), 8.55(\mathrm{~d}, 1 \mathrm{H}, \mathrm{J}=4.5 \mathrm{~Hz})$ ppm; ${ }^{13} \mathrm{C}$ NMR $\left(75 \mathrm{MHz}, \mathrm{CDCl}_{3}\right) \delta 22.83,42.18,56.19,60.07$, $118.00,121.91,122.44,134.05,136.64,149.12,160.49$ ppm; FT-IR (film, $\left.\mathrm{cm}^{-1}\right) \vee$ 3211, 2981, 2849, 1655, 809; ESI-MS: $253.2\left(\mathrm{M}^{+}+\mathrm{H}\right), 288.2\left(\mathrm{M}^{+}+\mathrm{Na}\right)$; HRMS (ESI) for $\left[\mathrm{C}_{13} \mathrm{H}_{20} \mathrm{~N}_{2} \mathrm{NaOS}\right]^{+}$: calcd. 275.1194, found 275.1191.

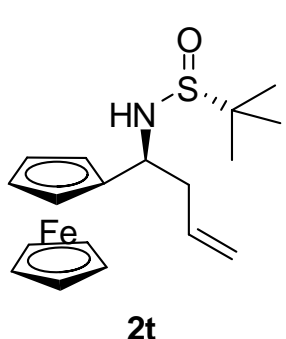

$2 t$

$[\alpha]_{\mathrm{D}}^{26}-103.5\left(\mathrm{c} 0.86, \mathrm{CHCl}_{3}\right)$ for $99 \% \mathrm{de}$; ${ }^{1} \mathrm{H}$ NMR $(300 \mathrm{MHz}$, $\left.\mathrm{CDCl}_{3}\right): \delta 1.23(\mathrm{~s}, 9 \mathrm{H}), 2.49-2.59(\mathrm{~m}, 1 \mathrm{H}), 2.75-2.83(\mathrm{~m}, 1 \mathrm{H}), 3.65$ $(\mathrm{d}, 1 \mathrm{H}, \mathrm{J}=5.7 \mathrm{~Hz}), 4.23(\mathrm{bs}, 8 \mathrm{H}), 4.29(\mathrm{~s}, 1 \mathrm{H}), 4.33(\mathrm{dd}, 1 \mathrm{H}, \mathrm{J}=$ 5.4, 6.9 Hz), 5.18- 5.23 (m, 2H), 5.77-5.91 (m, 1H) ppm; ${ }^{13} \mathrm{C} \mathrm{NMR}$ $\left(100 \mathrm{MHz}, \mathrm{CDCl}_{3}\right) \delta 22.78,42.06,53.81,55.87,65.94,67.41$, 67.94, 68.04, 68.66, 90.79, 119.14, 134.49 ppm; FT-IR (film, cm-1) v 3241, 2956, 2873, 1648, 1063, 831; ESI-MS: $360.1\left(\mathrm{M}^{+}+\mathrm{H}\right)$; HRMS (ESI) for $\left[\mathrm{C}_{18} \mathrm{H}_{25} \mathrm{FeN} \mathrm{Na} \mathrm{OS}\right]^{+}$: calcd. 382.0904, found 382.0902.

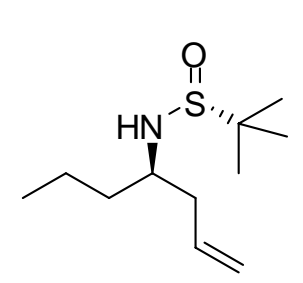

$[\alpha]_{\mathrm{D}}{ }^{26}-16.2\left(\mathrm{c} 1.00, \mathrm{CHCl}_{3}\right)$ for $85 \% \mathrm{de} ;{ }^{1} \mathrm{H}$ NMR $(500 \mathrm{MHz}$, $\left.\mathrm{CDCl}_{3}\right): \delta 0.91(\mathrm{t}, 3 \mathrm{H}, \mathrm{J}=7.4 \mathrm{~Hz}), 1.20(\mathrm{~s}, 9 \mathrm{H}), 1.35-1.48(\mathrm{~m}, 4 \mathrm{H})$, 2.30-2.41 (m, 2H), $3.20(\mathrm{~d}, 1 \mathrm{H}, \mathrm{J}=5.9 \mathrm{~Hz}), 3.30-3.34(\mathrm{~m}, 1 \mathrm{H})$, 5.79-5.82 (m, 2H), 5.77-5.82 (m, 1H) ppm; ${ }^{13} \mathrm{C}$ NMR $(125 \mathrm{MHz}$, $\left.\mathrm{CDCl}_{3}\right) \delta 13.91,18.72,22.61,37.20,40.44,54.72,55.70,118.75$, 2u $\quad 134.20$ ppm; FT-IR (film, $\left.\mathrm{cm}^{-1}\right) \vee 3230,3071,2959,2869,1642$, 1369, 1061, 913; ESI-MS (m/z, \%): $204.2\left(\mathrm{M}^{+}+\mathrm{H}\right)$; HRMS (EI) for $\mathrm{C}_{11} \mathrm{H}_{23} \mathrm{NOS}$ : calcd. 217.1500, found 217.1505.

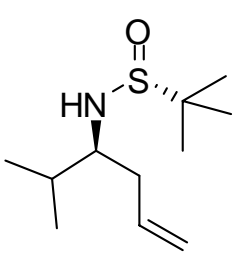

2v

$[\alpha]_{\mathrm{D}}{ }^{26}-48.5\left(\mathrm{c} 1.03, \mathrm{CHCl}_{3}\right)$ for $93 \% \mathrm{de} ;{ }^{1} \mathrm{H}$ NMR $(500 \mathrm{MHz}$, $\left.\mathrm{CDCl}_{3}\right): \delta 0.91(\mathrm{dd}, 6 \mathrm{H}, \mathrm{J}=34.1,7.5 \mathrm{~Hz}), 1.22(\mathrm{~s}, 9 \mathrm{H}), 1.86-1.90(\mathrm{~m}$, $1 \mathrm{H})$, 2.28-2.39 (m, 2H), 3.14-3.16 (m, $1 \mathrm{H}), 5.14-5.17(\mathrm{~m}, 2 \mathrm{H})$, 5.76-5.84 (m, 1H) ppm; ${ }^{13} \mathrm{C}$ NMR (125 MHz, $\left.\mathrm{CDCl}_{3}\right) \delta 17.81,18.37$, 
22.72, 31.00, 36.98, 55.88, 59.94, 118.58, 134.72 ppm; FT-IR (film, $\mathrm{cm}^{-1}$ ) v 3234, 3076, 2956, 2926, 2871, 1640, 1465, 1389, 1056, 908; ESI-MS (m/z, \%): 218.2 $\left(\mathrm{M}^{+}+\mathrm{H}\right)$; HRMS (EI) for $\mathrm{C}_{11} \mathrm{H}_{23} \mathrm{NOS}$ : calcd. 217.1500, found 217.1489.

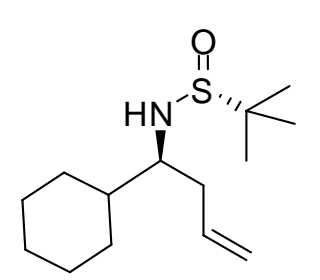

$2 w$

$[\alpha]_{\mathrm{D}}{ }^{26}-30.1\left(\mathrm{c} 1.02, \mathrm{CHCl}_{3}\right)$ for $93 \% \mathrm{de} ;{ }^{1} \mathrm{H}$ NMR $(500 \mathrm{MHz}$, $\left.\mathrm{CDCl}_{3}\right): \delta$ 0.98-1.26 (br, m, 6H), $1.21(\mathrm{~s}, 9 \mathrm{H}), 1.66-1.69(\mathrm{~m}, 1 \mathrm{H})$, 1.74-1.77 (m, 5H), 3.13-3.21 (m, 2H), 5.14-5.17 (m, 2H), 5.76-5.82 (m, 1H) ppm; ${ }^{13} \mathrm{C}$ NMR $\left(125 \mathrm{MHz}, \mathrm{CDCl}_{3}\right) \delta 22.74$, $26.24,26.28,26.54,28.54,29.10,31.19,41.24,55.95,59.74$, 118.63, 134.65 ppm; FT-IR (film, $\left.\mathrm{cm}^{-1}\right) \vee 3244,3071,3977,2925$, 2853, 1449, 1055, 909, 505; ESI-MS (m/z, \%): $258.2(\mathrm{M}++\mathrm{H})$; HRMS (EI) for $\mathrm{C}_{14} \mathrm{H}_{27} \mathrm{NOS}$ : calcd. 257.1813, found 257.1824.

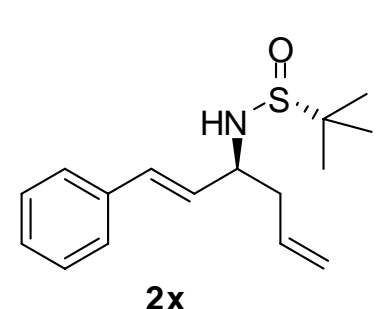

$2 x$

$[\alpha]_{\mathrm{D}}{ }^{26}-136.2\left(\mathrm{c} 1.05, \mathrm{CHCl}_{3}\right)$ for $93 \% \mathrm{de} ;{ }^{1} \mathrm{H} \mathrm{NMR}(500 \mathrm{MHz}$, $\left.\mathrm{CDCl}_{3}\right): \delta 1.22(\mathrm{~s}, 9 \mathrm{H}), 2.38-2.52(\mathrm{~m}, 2 \mathrm{H}), 3.48(\mathrm{~d}, 1 \mathrm{H}, \mathrm{J}=2.2$ $\mathrm{Hz}), 4.06-4.08(\mathrm{~m}, 1 \mathrm{H}), 5.17-5.21(\mathrm{~m}, 2 \mathrm{H}), 5.78-5.83(\mathrm{~m}, 1 \mathrm{H})$, 6.00-6.05 (m, 1H), $6.60(\mathrm{~d}, 1 \mathrm{H}, \mathrm{J}=15.9 \mathrm{~Hz}), 7.22-7.38(\mathrm{~m}$, $5 \mathrm{H}) \mathrm{ppm} ;{ }^{13} \mathrm{C} \mathrm{NMR}\left(125 \mathrm{MHz}, \mathrm{CDCl}_{3}\right) \delta 22.58,41.23,55.49$, $55.83,119.10,126.44,127.66,128.49,129.84,132.28,133.71$.

$136.51 \mathrm{ppm}$; FT-IR $\left(\mathrm{KBr}, \mathrm{cm}^{-1}\right) \vee 3199,3073,2976,2867,1640,1053,964,910,752$. 694; ESI-MS (m/z, \%): $278.2\left(\mathrm{M}^{+}+\mathrm{H}\right)$; HRMS (EI) for $\mathrm{C}_{16} \mathrm{H}_{23} \mathrm{NOS}$ : calcd. 277.1500, found 277.1509 .

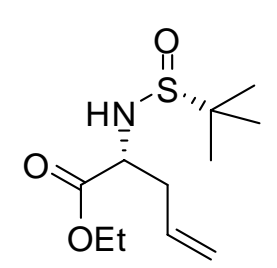

$2 y$

$[\alpha]_{\mathrm{D}}{ }^{26}-94.3\left(\mathrm{c} 1.00, \mathrm{CHCl}_{3}\right)$ for $96 \% \mathrm{de} ;{ }^{1} \mathrm{H} \mathrm{NMR}\left(500 \mathrm{MHz}, \mathrm{CDCl}_{3}\right)$ : $\delta 1.25(\mathrm{~s}, 9 \mathrm{H}), 1.29(\mathrm{t}, 3 \mathrm{H}, \mathrm{J}=7.13 \mathrm{~Hz}), 2.49-2.56(\mathrm{~m}, 2 \mathrm{H}), 4.03(\mathrm{dd}$, $1 \mathrm{H}, \mathrm{J}=6.23,5.93 \mathrm{~Hz}), 4.14(\mathrm{~d}, 1 \mathrm{H}, \mathrm{J}=5.96 \mathrm{~Hz}), 4.23(\mathrm{q}, 2 \mathrm{H}, \mathrm{J}=$ $7.12 \mathrm{~Hz}), 5.10-5.13(\mathrm{~m}, 2 \mathrm{H}), 5.70-5.76(\mathrm{~m}, 1 \mathrm{H}) \mathrm{ppm} ;{ }^{13} \mathrm{C}$ NMR $(125$ $\left.\mathrm{MHz}, \mathrm{CDCl}_{3}\right) \delta 14.10,22.58,38.08,56.07,56.88,61.67,118.71$, 132.37, 172.40 ppm; FT-IR (KBr, cm-1) :v 2817, 1649, 1053, 964, 903, 742; $\quad$ ESI-MS: $248.2 \quad\left(\mathrm{M}^{+}+\mathrm{H}\right), \quad 270.1\left(\mathrm{M}^{+}+\mathrm{Na}^{+}\right)$; HRMS (ESI) for $\left[\mathrm{C}_{11} \mathrm{H}_{21} \mathrm{NNaO}_{3} \mathrm{~S}\right]^{+}\left(\mathrm{M}^{+}+\mathrm{Na}\right)$ : calcd. 270.1133, found 270.1134 .

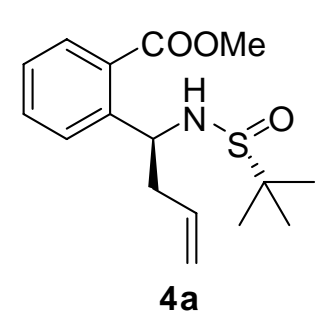

$[\alpha]_{D}^{26}-85.67\left(\mathrm{c} 1.07, \mathrm{CHCl}_{3}\right)$ for $99 \%$ de; ${ }^{1} \mathrm{H}$ NMR $(500 \mathrm{MHz}$, $\left.\mathrm{CDCl}_{3}\right): \delta 1.19(\mathrm{~s}, 9 \mathrm{H}), 2.47-2.53(\mathrm{~m}, 1 \mathrm{H}), 2.76-2.81(\mathrm{~m}, 1 \mathrm{H})$, $3.91(\mathrm{~s}, 3 \mathrm{H}), 4.01(\mathrm{~d}, 1 \mathrm{H}, \mathrm{J}=3.1 \mathrm{~Hz}), 5.15-5.19(\mathrm{~m}, 2 \mathrm{H})$, 5.27-5.30 $(\mathrm{m}, 1 \mathrm{H}), \quad 5.77-5.84(\mathrm{~m}, 1 \mathrm{H}), \quad 7.30-7.33(\mathrm{~m}, 1 \mathrm{H})$, 7.50-7.52 (m, $1 \mathrm{H}), 7.55-7.57(\mathrm{~m}, 1 \mathrm{H}), 7.86-7.88(\mathrm{~m}, 1 \mathrm{H}) \mathrm{ppm}$; ${ }^{13} \mathrm{C}$ NMR $\left(125 \mathrm{MHz}, \mathrm{CDCl}_{3}\right) \delta 22.49,42.49,52.13,53.97,55.67$, $118.75,126.99,128.10,129.39,130.48,131.82,134.47,143.63,167.73$ ppm; FT-IR $\left(\mathrm{KBr}, \mathrm{cm}^{-1}\right)$ v: 3199, 3073, 2976, 2867, 1640, 1053, 964, 910, 752. 694; ESI-MS: $310.1\left(\mathrm{M}^{+}+\mathrm{H}\right)$, 332.1( $\left(\mathrm{M}^{+}+\mathrm{Na}^{+}\right)$; HRMS (ESI) for $\mathrm{C}_{16} \mathrm{H}_{24} \mathrm{NOS}\left(\mathrm{M}^{+}+\mathrm{H}\right)$ : calcd. 310.1477, found 310.1467 . 


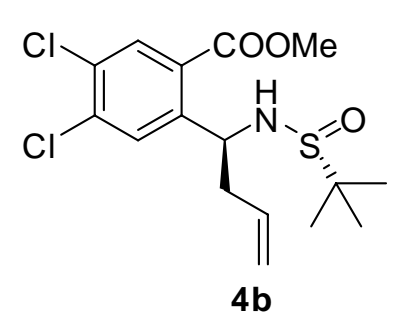

$[\alpha]_{\mathrm{D}}{ }^{26}-123.5$ (c $\left.1.03, \mathrm{CHCl}_{3}\right)$ for $99 \% \mathrm{de} ;{ }^{1} \mathrm{H}$ NMR $(300$ $\left.\mathrm{MHz}, \mathrm{CDCl}_{3}\right): \delta 1.22(\mathrm{~s}, 9 \mathrm{H}), 2.30-2.40(\mathrm{~m}, 1 \mathrm{H}), 2.69-2.77$ $(\mathrm{m}, 1 \mathrm{H}), 3.88(\mathrm{~s}, 4 \mathrm{H}), 5.12-5.18(\mathrm{~m}, 2 \mathrm{H}), 5.24-5.34(\mathrm{~m}, 1 \mathrm{H})$, 5.69-5.83 (m, 1H), $7.66(\mathrm{~s}, 1 \mathrm{H}), 7.96(\mathrm{~s}, 1 \mathrm{H}) \mathrm{ppm} ;{ }^{13} \mathrm{C} \mathrm{NMR}$ $\left(75 \mathrm{MHz}, \mathrm{CDCl}_{3}\right) \delta 22.56,42.43,52.65,55.96,119.58$, $128.75,130.52,131.28,132.58,133.97,136.65,144.34$, 165.59 ppm; FT-IR $\left(\mathrm{KBr}, \mathrm{cm}^{-1}\right) \vee 3195,2969,1645,1059$, 817; ESI-MS: 378.0 $\left(\mathrm{M}^{+}+\mathrm{H}\right)$; HRMS (ESI) for $\left[\mathrm{C}_{16} \mathrm{H}_{21} \mathrm{Cl}_{2} \mathrm{NNaO}_{3} \mathrm{~S}\right]^{+}$: calcd. 400.0511, found 400.0512 .

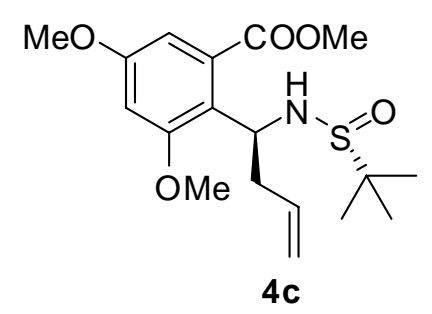

$[\alpha]_{\mathrm{D}}{ }^{26}-21.9$ (c 1.45, $\left.\mathrm{CHCl}_{3}\right)$ for $99 \%$ de; ${ }^{1} \mathrm{H}$ NMR $(400$ $\left.\mathrm{MHz}, \mathrm{CDCl}_{3}\right): \delta 1.10$ (s, 9H), 2.65-2.75 (m, 1H), 2.82-2.90 $(\mathrm{m}, 1 \mathrm{H}), 3.82(\mathrm{~s}, 3 \mathrm{H}), 3.86(\mathrm{~s}, 3 \mathrm{H}), 3.90(\mathrm{~s}, 3 \mathrm{H}), 4.59(\mathrm{~d}, 1 \mathrm{H}$, $\mathrm{J}=9.3 \mathrm{~Hz}), 4.95-4.97(\mathrm{~m}, 2 \mathrm{H}), 5.01-5.12(\mathrm{~m}, 1 \mathrm{H})$, 5.71-5.75 (m, 1H), $6.60(\mathrm{~d}, 1 \mathrm{H}, \mathrm{J}=2.4 \mathrm{~Hz}), 6.80(\mathrm{~d}, 1 \mathrm{H}, \mathrm{J}=$ $2.4 \mathrm{~Hz}) \mathrm{ppm} ;{ }^{13} \mathrm{C}$ NMR $(100 \mathrm{MHz}, \mathrm{CDCl} 3) \delta 22.22,41.04$, 52.22, 55.24, 55.51, 55.60, 102.10, 105.24, 116.71, 123.30, 131.78, 135.21, 158.40, 158.88, 168.73 ppm; FT-IR (film, cm-1) v 3186, 3080, 2981, 1649, 1051, 910, 752; ESI-MS: $370.2\left(\mathrm{M}^{+}+\mathrm{H}\right)$; HRMS (ESI) for $\left[\mathrm{C}_{18} \mathrm{H}_{27} \mathrm{NNaO}_{5} \mathrm{~S}\right]^{+}$: calcd. 392.1508, found 392.1510 .

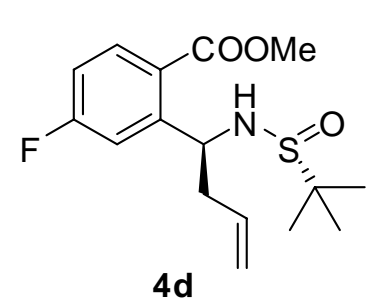

$[\alpha]_{\mathrm{D}}{ }^{26}-99.5\left(\mathrm{c} 1.00, \mathrm{CHCl}_{3}\right)$ for $99 \% \mathrm{de} ;{ }^{1} \mathrm{H}$ NMR $(300 \mathrm{MHz}$, $\left.\mathrm{CDCl}_{3}\right): \delta 1.22(\mathrm{~s}, 9 \mathrm{H}), 2.37-2.47(\mathrm{~m}, 1 \mathrm{H}), 2.75-2.84(\mathrm{~m}, 1 \mathrm{H})$, $3.91(\mathrm{~s}, 3 \mathrm{H}), 3.94(\mathrm{~d}, 1 \mathrm{H}, \mathrm{J}=2.4 \mathrm{~Hz}), 5.17-5.22(\mathrm{~m}, 2 \mathrm{H})$, 5.37-5.42 (m, 1H), 5.75-5.88 (m, 1H), 6.97-7.03 (m, 1H), $7.29(\mathrm{dd}, 1 \mathrm{H}, \mathrm{J}=3.0,10.8 \mathrm{~Hz}), 7.96(\mathrm{dd}, 1 \mathrm{H}, \mathrm{J}=6.3,9.0 \mathrm{~Hz})$ ppm; ${ }^{19} \mathrm{~F}$ NMR: $\delta-106.12(\mathrm{dd}, \mathrm{J}=6.3,16.8 \mathrm{~Hz}, 1 \mathrm{~F}) ;{ }^{13} \mathrm{C}$ NMR $\left(75 \mathrm{MHz}, \mathrm{CDCl}_{3}\right) \delta 22.56,42.51,52.29,53.14,55.88,114.06,114.35,115.06,115.36$, 119.34, 125.23, 133.44, 133.54, 134.21, 147.87, 147.97, 166.55, 166.60 ppm; FT-IR $\left(\mathrm{KBr}, \mathrm{cm}^{-1}\right) \vee 3201,2868,1650,1049,818$; ESI-MS:328.1 $\left(\mathrm{M}^{+}+\mathrm{H}\right)$; HRMS (ESI) for $\left[\mathrm{C}_{16} \mathrm{H}_{22} \mathrm{FNNaO}_{3} \mathrm{~S}\right]^{+}$: calcd. 350.1197, found 350.1199.

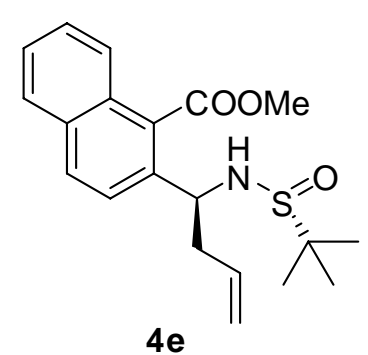

$[\alpha]_{\mathrm{D}}{ }^{26}-86.2\left(\mathrm{c} 1.03, \mathrm{CHCl}_{3}\right)$ for $99 \%$ de; ${ }^{1} \mathrm{H}$ NMR $(300 \mathrm{MHz}$, $\left.\mathrm{CDCl}_{3}\right): \delta 1.16(\mathrm{~s}, 9 \mathrm{H}), 2.51-2.76(\mathrm{~m}, 2 \mathrm{H}), 3.85(\mathrm{~s}, 1 \mathrm{H}), 4.08(\mathrm{~s}$, $1 \mathrm{H}), 4.67-4.71(\mathrm{~m}, 1 \mathrm{H}), 5.15-5.21(\mathrm{~m}, 2 \mathrm{H}), 5.70-5.84(\mathrm{~m}, 1 \mathrm{H})$, 7.48-7.60 (m, 3H), 7.80-7.91 (m, 3H) ppm; ${ }^{13} \mathrm{C}$ NMR $(75 \mathrm{MHz}$, $\left.\mathrm{CDCl}_{3}\right) \delta 22.56,42.70,52.62,54.72,55.79,119.43,124.26$, $125.21,126.56,127.32,128.11,129.63,130.14,130.82,132.65$, 133.99, 136.85, 169.50 ppm; FT-IR (film, cm-1) v 3194, 2980, 1649, 1049, 809 ; ESI-MS: $360.1\left(\mathrm{M}^{+}+\mathrm{H}\right)$; HRMS (ESI) for $\left[\mathrm{C}_{20} \mathrm{H}_{25} \mathrm{NNaO}_{3} \mathrm{~S}\right]^{+}$: calcd. 382.1453 , found 382.1456 .

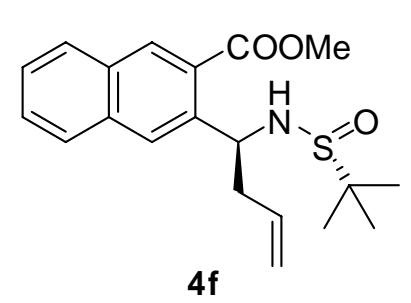

$[\alpha]_{\mathrm{D}}{ }^{26}-131.9\left(\mathrm{c} 1.00, \mathrm{CHCl}_{3}\right)$ for $99 \%$ de; ${ }^{1} \mathrm{H}$ NMR $(300$ $\left.\mathrm{MHz}, \mathrm{CDCl}_{3}\right): \delta 1.22(\mathrm{~s}, 9 \mathrm{H}), 2.56-2.66(\mathrm{~m}, 1 \mathrm{H}), 2.87-2.96$ $(\mathrm{m}, 1 \mathrm{H}), 3.97(\mathrm{~s}, 3 \mathrm{H}), 4.20(\mathrm{~d}, 1 \mathrm{H}, \mathrm{J}=3.6 \mathrm{~Hz}), 5.15-5.23(\mathrm{~m}$, 
2H), 5.35-5.40 (m, 1H), 5.78-5.91 (m, 1H), 7.48-7.60 (m, 2H), $7.81(\mathrm{~d}, 1 \mathrm{H}, \mathrm{J}=7.5$ $\mathrm{Hz}), 7.88$ (d, 1H, J = 8.1 Hz), 7.99 (s, 1H), 8.49 (s, 1H) ppm; ${ }^{13} \mathrm{C}$ NMR $(75 \mathrm{MHz}$, $\left.\mathrm{CDCl}_{3}\right) \delta 22.67,42.73,52.42,54.93,55.88,118.80,126.77,127.34,127.66,127.74$, 128.45, 128.70, 131.52, 132.47, 134.67, 134.84, 139.44, 168.03 ppm; FT-IR (film, cm-1) v 3204, 2976, 2863, 1643, 1053, 816; ESI-MS: $360.2\left(\mathrm{M}^{+}+\mathrm{H}\right)$; HRMS (ESI) for $\left[\mathrm{C}_{20} \mathrm{H}_{25} \mathrm{NNaO}_{3} \mathrm{~S}\right]^{+}$: calcd. 382.1453, found 382.1455 .<smiles>C=CC[C@H](NS(=O)C(C)(C)C)c1cc(Br)ccc1C(=O)OCC</smiles>

$[\alpha]_{\mathrm{D}}{ }^{26}-111.1\left(\mathrm{c} 1.00, \mathrm{CHCl}_{3}\right)$ for $99 \% \mathrm{de} ;{ }^{1} \mathrm{H}$ NMR $(300$ $\left.\mathrm{MHz}, \mathrm{CDCl}_{3}\right): \delta 1.08(\mathrm{~s}, 9 \mathrm{H}), 1.40(\mathrm{t}, 7.2 \mathrm{~Hz}), 2.35-2.46(\mathrm{~m}$, $1 \mathrm{H}), 2.76-2.84(\mathrm{~m}, 1 \mathrm{H}), 3.91(\mathrm{~d}, 1 \mathrm{H}, \mathrm{J}=2.7 \mathrm{~Hz}), 4.37(\mathrm{t}, 2 \mathrm{H}$, $\mathrm{J}=7.2 \mathrm{~Hz}), 5.18-5.19$ (d-like, 2H), 5.22 (s-like, 1H), 5.29-5.34 (m, 1H), 5.74-5.88 (m, 1H), 7.43-7.47 (m, 1H), 7.74-7.78 (m, 2H) ppm; ${ }^{13} \mathrm{C}$ NMR $\left(75 \mathrm{MHz}, \mathrm{CDCl}_{3}\right) \delta 14.23$, 22.59, 42.64, 53.09, 55.92, 61.53, 119.43, 126.84, 128.56, 130.27, 131.59, 132.29, 134.23, 146.11, 166.53 ppm; FT-IR (film, cm-1) v 3211, 2982, 2854, 1681, 1047, 821 ; ESI-MS: $402.1\left(\mathrm{M}^{+}+\mathrm{H}\right)$; HRMS (ESI) for $\left[\mathrm{C}_{17} \mathrm{H}_{24} \mathrm{BrNNaO}_{3} \mathrm{~S}\right]^{+}$: calcd.424.0558, found 424.0556 .

\section{General procedure for the synthesis of chiral 3-allylsubstituted} isoindolinones.<smiles></smiles><smiles>CO[Mg]C(C)C(C)(C)C</smiles><smiles>[R]c1c([R])c([R])c2c(c1[R])C(=O)NC2CC=C</smiles>

To a solution of compound $4(0.2 \mathrm{mmol})$ in methanol $(5 \mathrm{~mL})$ was added $0.2 \mathrm{~mL}$ of $12 \mathrm{~N} \mathrm{HCl}$ aqueous solution at room temperature. The resultant mixture was then stirred at $\mathrm{rt}$ for 0.5 to 1 hour. After concentrated, the residue was dissolved in $20 \mathrm{~mL}$ $\mathrm{CH}_{2} \mathrm{Cl}_{2}$ and $5 \mathrm{~mL}$ of saturated aqueous $\mathrm{NaHCO}_{3}$ was added. After 1 hour, the mixture is extracted with dichloromethane, washed with brine. The organic layer was dried and concentrated under vacuum. The residue was purified by silica gel column chromatography to afford chiral 3-allylsubstituted isoindolinones $\mathbf{5}$ as a solid.

\section{Characterization data of the obtained chiral 3-allylsubstituted isoindolinones.}<smiles>C=CCC1NC(=O)c2ccccc21</smiles>

$5 a$

99\% yield. $[\alpha]_{\mathrm{D}}{ }^{26}-78.1$ (c $1.20, \mathrm{CH}_{2} \mathrm{Cl}_{2}$ ) for $99.4 \%$ ee [Lit: $[\alpha]_{\mathrm{D}}-70$ (c 0.63, $\mathrm{CH}_{2} \mathrm{Cl}_{2}$ ) for $99 \%$ ee, S, Org. Lett. 2005, 7, 95]; ${ }^{1} \mathrm{H}$ NMR (300 $\left.\mathrm{MHz}, \mathrm{CDCl}_{3}\right): \delta 2.28-2.38(\mathrm{~m}, 1 \mathrm{H}), 2.70-2.79(\mathrm{~m}, 1 \mathrm{H}), 4.66(\mathrm{dd}, 1 \mathrm{H}, \mathrm{J}$ $=8.1,4.5 \mathrm{~Hz}), 5.16-5.21(\mathrm{~m}, 2 \mathrm{H}), 5.75-5.89(\mathrm{~m}, 1 \mathrm{H}), 6.82(\mathrm{~s}, 1 \mathrm{H})$, 7.45-7.60 (m, 3H), $7.86(\mathrm{~d}, 1 \mathrm{H}, \mathrm{J}=13.8 \mathrm{~Hz}) \mathrm{ppm} ;{ }^{13} \mathrm{C}$ NMR $(100$ 
$\left.\mathrm{MHz}, \mathrm{CDCl}_{3}\right) \delta 38.86,56.18,119.07,122.48,123.66,128.11,131.65,132.04,132.87$, 146.92, 171.03 ppm; FT-IR $\left(\mathrm{KBr}, \mathrm{cm}^{-1}\right) \vee$ 3151, 1690, 1682, 1459, 1386; ESI-MS: $174.1\left(\mathrm{M}^{+}+\mathrm{H}\right)$; HRMS (ESI) for $\left[\mathrm{C}_{11} \mathrm{H}_{11} \mathrm{NNaO}\right]^{+}$: calcd. 196.0738, found 196.0729. HPLC (Chiralpak OJ-H column, 70:30 hexane/2-propanol; 0.7ml/min; 254nm; Retention time: $6.8 \mathrm{~min}$ for $(S)-6 \mathrm{a}, 8.2 \mathrm{~min}$ for $(R)-6 \mathrm{a})$;

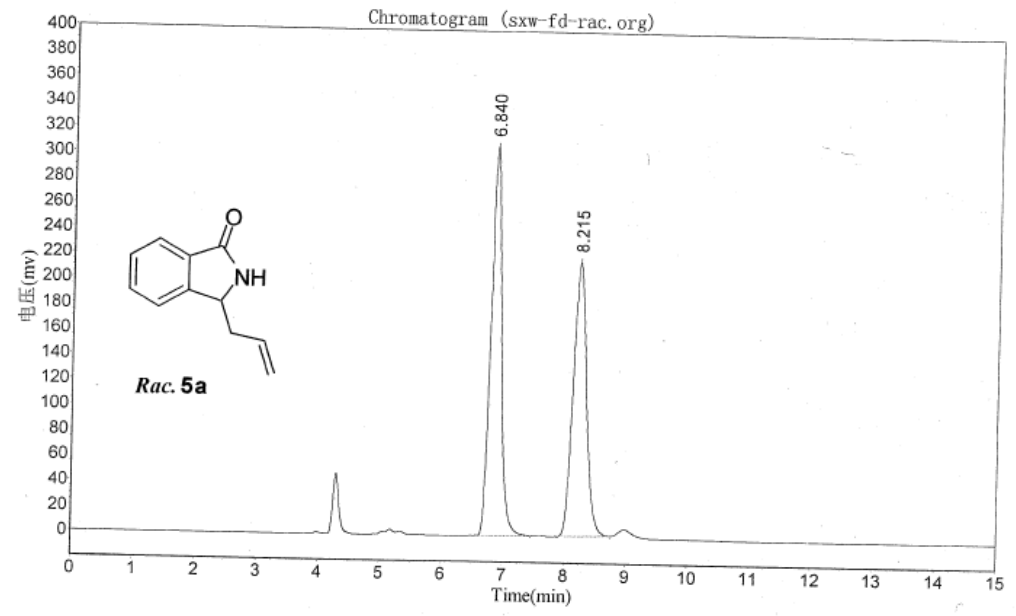

Sample Description:
OD-H $7 / 3 \quad 0.7 \mathrm{ml} 1$ min $214 \mathrm{~nm}$
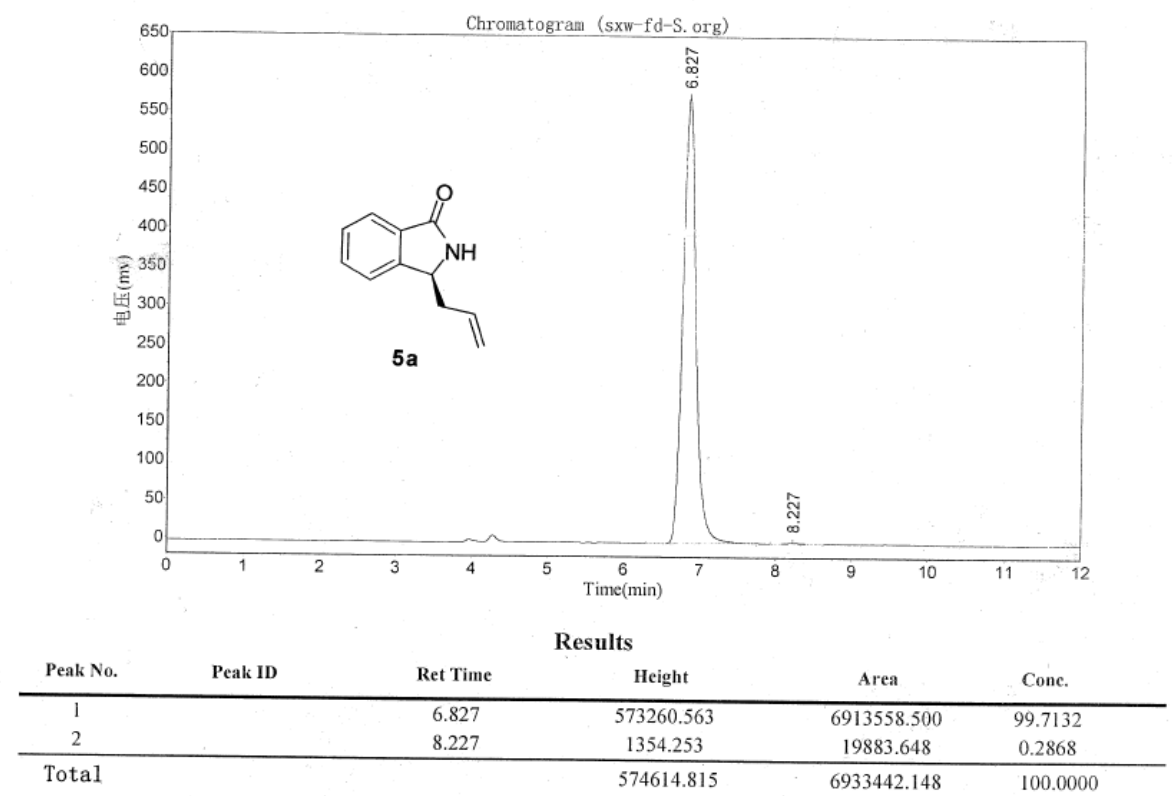<smiles>C=CC[C@@H]1NC(=O)c2cc(Cl)c(Cl)cc21</smiles>

$5 b$

96\% yield. $[\alpha]_{\mathrm{D}}{ }^{26}-102.0$ (c 1.15, $\mathrm{CHCl}_{3}$ ); ${ }^{1} \mathrm{H}$ NMR $(400 \mathrm{MHz}$, $\left.\mathrm{CDCl}_{3}\right): \delta$ 2.30-2.70 (m, 2H), 4.61-4.64 (m, $\left.1 \mathrm{H}\right)$, 5.12-5.16 $(\mathrm{m}$, 2H), 5.70-5.85 (m, 1H), $7.54(\mathrm{~s}, 1 \mathrm{H}), 7.85(\mathrm{~s}, 1 \mathrm{H}), 8.45(\mathrm{~s}, 1 \mathrm{H})$ ppm; ${ }^{13} \mathrm{C}$ NMR $\left(100 \mathrm{MHz}, \mathrm{CDCl}_{3}\right) \delta 38.65,56.04,119.90,124.96$, $125.62,132.18,132.25,133.14,136.35,146.20,169.24$ ppm; FT-IR $\left(\mathrm{KBr}, \mathrm{cm}^{-1}\right) \vee$ 3160, 1685, 1690, 1452, 1379; ESI-MS: 242.0 $\left(\mathrm{M}^{+}+\mathrm{H}\right)$; HRMS (ESI) for $\left[\mathrm{C}_{11} \mathrm{H}_{9} \mathrm{Cl}_{2} \mathrm{NnaO}\right]^{+}$: calcd. 263.9959, found 263.9948. 
<smiles>C=CC[C@H]1NC(=O)c2cc(OC)cc(OC)c21</smiles>

$5 \mathrm{c}$

$88 \%$ yield. $[\alpha]_{\mathrm{D}}{ }^{26}-108.9\left(\mathrm{c} 1.01, \mathrm{CHCl}_{3}\right) ;{ }^{1} \mathrm{H}$ NMR $(400 \mathrm{MHz}$, $\left.\mathrm{CDCl}_{3}\right): \delta$ 2.23-2.31 (m, 1H), 2.96-3.00 (m, 1H), 3.86 (d-like, $6 \mathrm{H}, \mathrm{J}=1.7 \mathrm{~Hz}), 4.61-4.63(\mathrm{~m}, 1 \mathrm{H}), 5.07-5.14(\mathrm{~m}, 2 \mathrm{H})$, 5.68-5.80 (m, 1H), $6.61(\mathrm{~d}, 1 \mathrm{H}, \mathrm{J}=2.0 \mathrm{~Hz}), 6.92(\mathrm{~d}, 1 \mathrm{H}, \mathrm{J}=2.0$ $\mathrm{Hz}), 7.25$ (s, 1H) ppm; ${ }^{13} \mathrm{C}$ NMR $\left(100 \mathrm{MHz}, \mathrm{CDCl}_{3}\right) \delta 37.08$, $54.89,55.54,55.89,97.71,102.86,118.68,127.42,133.57$,

134.57, 155.66, 161.89, 170.83 ppm; FT-IR (film, cm-1) v 3159, 1699, 1687, 1462; ESI-MS: $224.1\left(\mathrm{M}^{+}+\mathrm{H}\right)$; HRMS (ESI) for $\left[\mathrm{C}_{13} \mathrm{H}_{15} \mathrm{~N} \mathrm{Na} \mathrm{O}\right]^{+}$: calcd. 256.0950, found 256.0959 .<smiles>C=CCC1NC(=O)c2ccc(F)cc21</smiles>

$5 d$

$99 \%$ yield. $[\alpha]_{\mathrm{D}}{ }^{26}-72.2$ (c $\left.1.38, \mathrm{CHCl}_{3}\right) ;{ }^{1} \mathrm{H}$ NMR $(400 \mathrm{MHz}$, $\left.\mathrm{CDCl}_{3}\right): \delta 2.38-2.44(\mathrm{~m}, 1 \mathrm{H}), 2.55-2.68(\mathrm{~m}, 1 \mathrm{H}), 4.61-4.64(\mathrm{~m}, 1 \mathrm{H})$, 5.13-5.17 (m, 2H), 5.69-5.82 (m, 1H), 7.11-7.16 (m, 2H), 7.78-7.92 $(\mathrm{m}, 2 \mathrm{H}) \mathrm{ppm} ;{ }^{13} \mathrm{C}$ NMR $\left(100 \mathrm{MHz}, \mathrm{CDCl}_{3}\right) \delta 38.87,56.03,109.95$, $110.19,116.00,116.23,119.61,125.83,125.92,128.23,132.54$, 149.50, 149.60, 164.08, 166.58, 170.20 ppm; FT-IR (film, $\left.\mathrm{cm}^{-1}\right) v$ 3164, 1693, 1053, 816; ESI-MS: $192.1\left(\mathrm{M}^{+}+\mathrm{H}\right)$; HRMS (ESI) for $\left[\mathrm{C}_{11} \mathrm{H}_{10} \mathrm{FN} \mathrm{Na} \mathrm{O}\right]^{+}$: calcd. 214.0644, found 214.0651.

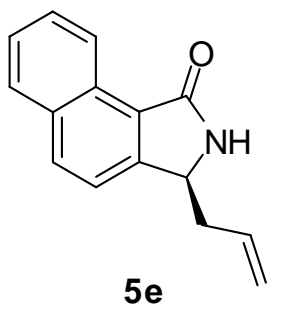

$89 \%$ yield. $[\alpha]_{\mathrm{D}}{ }^{26}-24.2$ (c 1.16, $\mathrm{CHCl}_{3}$ ); ${ }^{1} \mathrm{H}$ NMR $(400 \mathrm{MHz}$, $\left.\mathrm{CDCl}_{3}\right): \delta 2.30-2.45(\mathrm{~m}, 1 \mathrm{H}), 2.72-2.82(\mathrm{~m}, 1 \mathrm{H}), 4.68-4.70(\mathrm{~m}, 1 \mathrm{H})$, 5.13-5.17 (m, 2H), 5.71-5.84 (m, 1H),7.47-8.00 (m, 6H), $9.21(\mathrm{~d}$, $1 \mathrm{H}, \mathrm{J}=0.8 \mathrm{~Hz}) \mathrm{ppm} ;{ }^{13} \mathrm{C} \mathrm{NMR}\left(100 \mathrm{MHz}, \mathrm{CDCl}_{3}\right) \delta 38.63,56.03$, $119.19,119.80,123.96,126.62,128.00,128.24,132.83,133.10$, 133.20, 147.78, 172.37 ppm; FT-IR (KBr, cm-1) v 3251, 1725, 1349, 1148, 1107, 785, 670, 544; ESI-MS: $224.2\left(\mathrm{M}^{+}+\mathrm{H}\right), 246.2\left(\mathrm{M}^{+}+\mathrm{Na}\right)$; HRMS (ESI) for $\left[\mathrm{C}_{15} \mathrm{H}_{13} \mathrm{~N} \mathrm{Na} \mathrm{O}\right]^{+}$: calcd. 246.0895, found 246.0884.

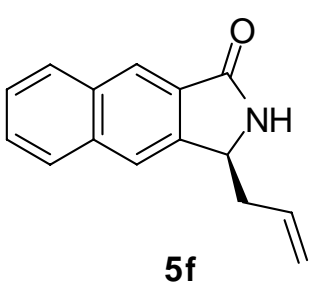

$92 \%$ yield. $[\alpha]_{\mathrm{D}}{ }^{26}-170.3$ (c $\left.0.95, \mathrm{CHCl}_{3}\right) ;{ }^{1} \mathrm{H}$ NMR $(400 \mathrm{MHz}$, $\left.\mathrm{CDCl}_{3}\right): \delta 2.47-2.54(\mathrm{~m}, 1 \mathrm{H}), 2.76-2.82(\mathrm{~m}, 1 \mathrm{H}), 4.81-4.84(\mathrm{~m}$, $1 \mathrm{H})$, 5.16-5.22 (m, 2H), 5.81-5.90 (m, 1H), 7.51-7.59 (m, 2H), $7.87(\mathrm{~s}, 1 \mathrm{H}), 7.91(\mathrm{~d}, 1 \mathrm{H}, \mathrm{J}=0.8 \mathrm{~Hz}), 8.00(\mathrm{~d}, 1 \mathrm{H}, \mathrm{J}=0.8 \mathrm{~Hz})$, $8.08(\mathrm{~s}, 1 \mathrm{H}), 8.39(\mathrm{~s}, 1 \mathrm{H}) \mathrm{ppm} ;{ }^{13} \mathrm{C}$ NMR $\left(100 \mathrm{MHz}, \mathrm{CDCl}_{3}\right) \delta$ $39.82,56.22,119.37,121.60,124.19,126.43,127.81,128.23$, 129.66, 130.26, 133.06, 133.09, 135.30, 142.11, 171.00 ppm; FT-IR (KBr, cm-1) v 3201, 1738, 1523, 1341, 1250, 1172, 1096, 666; ESI-MS: $224.2\left(\mathrm{M}^{+}+\mathrm{H}\right)$, 246.2( $\left(\mathrm{M}^{+}+\mathrm{Na}\right)$; HRMS (ESI) for $\left[\mathrm{C}_{15} \mathrm{H}_{13} \mathrm{NNaO}\right]^{+}$: calcd. 246.0895, found 246.0889.<smiles>C=CC[C@H]1NC(=O)c2ccc(Br)cc21</smiles>

$5 g$

95\% yield. $[\alpha]_{\mathrm{D}}{ }^{26}-48.6$ (c $\left.1.00, \mathrm{CHCl}_{3}\right) ;{ }^{1} \mathrm{H}$ NMR $(400 \mathrm{MHz}$, $\left.\mathrm{CDCl}_{3}\right): \delta 2.34-2.42(\mathrm{~m}, 1 \mathrm{H}), 2.61-2.68(\mathrm{~m}, 1 \mathrm{H}), 4.63(\mathrm{t}-$ like, $1 \mathrm{H})$, 5.13-5.17 (m, 2H), 5.70-5.79 (m, 1H), 7.59-7.62 (m, 2H), 7.67-7.69 (m, 1H), 8.04 (s, 1H) ppm; ${ }^{13} \mathrm{C}$ NMR (100 MHz, CDCl3) $\delta 38.80,56.08,119.70,125.29,126.12,126.62,131.21,131.80$, 
132.51, 148.85, 170.32 ppm; FT-IR $\left(\mathrm{KBr}, \mathrm{cm}^{-1}\right) \vee 3155,1686,1678,1455,1374$; ESI-MS: $252.0\left(\mathrm{M}^{+}+\mathrm{H}\right)$; HRMS (ESI) for $\left[\mathrm{C}_{11} \mathrm{H}_{10} \mathrm{BrNNaO}\right]^{+}$: calcd. 273.9843 , found 273.9841 .

\section{The synthesis and HPLC of D-allylglycine}

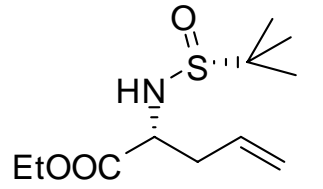

1) $6 \mathrm{~N} \mathrm{HCl}, 90^{\circ} \mathrm{C}$

2) Propylene oxide<smiles>C=CC[C@H](N)C(=O)O</smiles>

2y $96 \%$ de

Compound $2 \mathbf{y}(1 \mathrm{mmol})$ was dissolved in $6 \mathrm{~N} \mathrm{HCl}(10 \mathrm{~mL})$ in a sealed tube heated at $90{ }^{\circ} \mathrm{C}$ for $4 \mathrm{~h}$. After the mixture was cooled to room temperature, The aqueous solution was evaporated under reduced pressure and the residue was dissolved in EtOH $(10 \mathrm{~mL})$. Propylene oxide $(3 \mathrm{~mL})$ was then added, and the mixture was stirred at room temperature for $1 \mathrm{~h}$ during which time white solids precipitated. The precipitate was collected by filtration, washed successively with cold $\mathrm{EtOH}$ and $\mathrm{Et}_{2} \mathrm{O}$, and air-dried to afford the desired D-allylglycine in $78 \%$ yield.<smiles>C=CC[C@H](N)C(=O)O</smiles>

$[\alpha]_{\mathrm{D}}^{26}+33.8\left(\right.$ c $\left.1.05, \mathrm{H}_{2} \mathrm{O}\right)$ for $95 \%$ ee $\left[\right.$ lit: $[\alpha]_{\mathrm{D}}+32.0\left(\mathrm{c} 1.0, \mathrm{H}_{2} \mathrm{O}\right)$, for $95 \%$ ee, J. Am. Chem. Soc. 2005, 127, 1066]; ${ }^{1} \mathrm{H}$ NMR (400 $\left.\mathrm{MHz}, \mathrm{D}_{2} \mathrm{O}\right)$ 2.50-2.70 (2H, br m), $3.79(1 \mathrm{H}$, br m), 5.24-5.29 $(2 \mathrm{H}$, m), 5.71-5.80 (1H, m); ppm; ${ }^{13} \mathrm{C}$ NMR (100 MHz, $\left.\mathrm{D}_{2} \mathrm{O}\right) \delta 34.85,53.97,120.55$, 131.24, 174.12 ppm; FT-IR $\left(\mathrm{KBr}, \mathrm{cm}^{-1}\right) \vee 2969,1518,1584,1400 ; \mathrm{m} / \mathrm{z}(\mathrm{EI}): 116$ (100\%), 66 (69). HPLC (Chiralpak CR column, $\mathrm{pH}=1 \mathrm{HClO}_{4} ; 0.7 \mathrm{ml} / \mathrm{min} ; 214 \mathrm{~nm}$; Retention time: $5.3 \mathrm{~min}$ for $(R)$-allylglycine, $8.3 \mathrm{~min}$ for $(S)$-allylglycine).
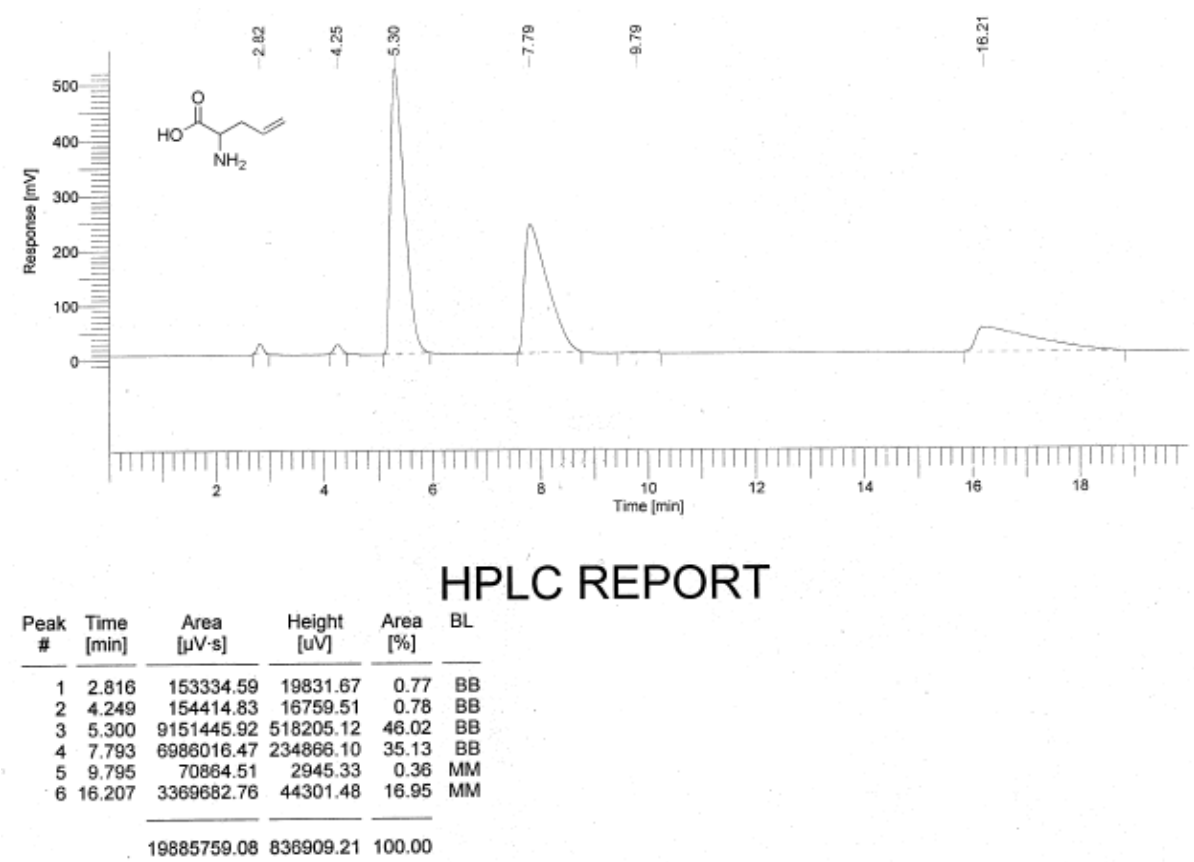


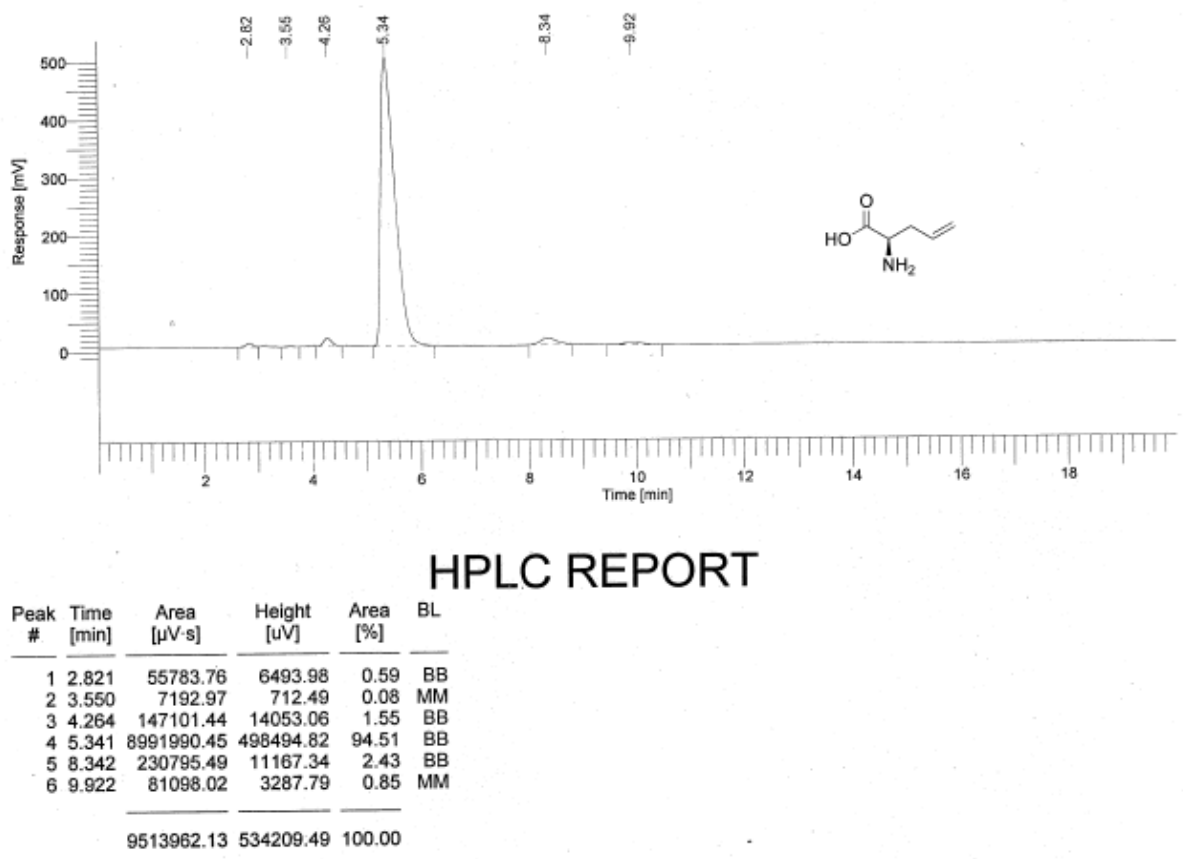




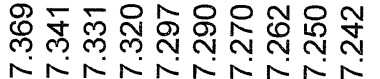

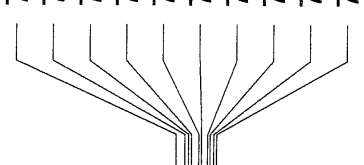

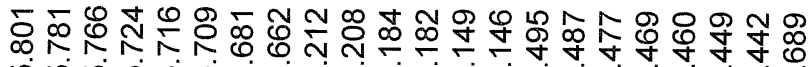

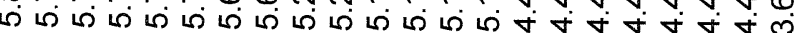

L

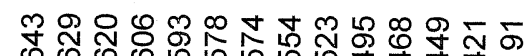

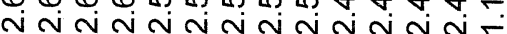

4

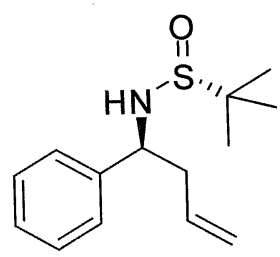

$2 a$

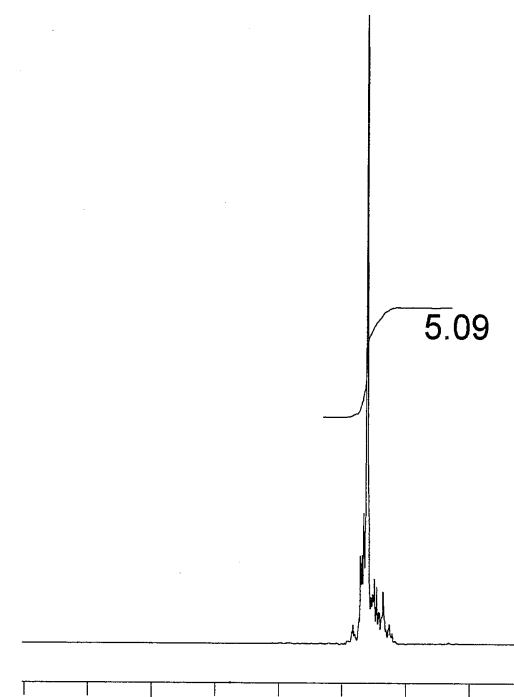

5.09

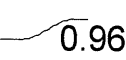

$\sqrt{2.07}$

$-1.00$

$-0.99$

$\sqrt{2.19}$ whth 


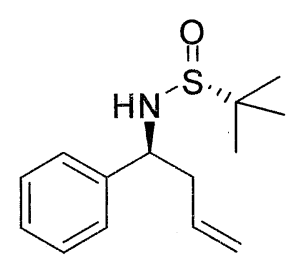

$2 a$

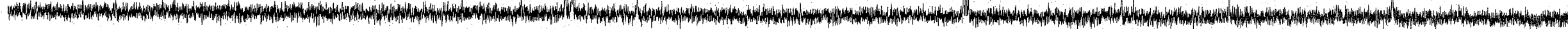




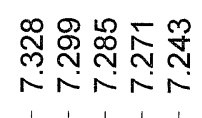

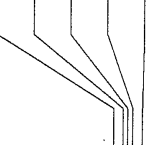

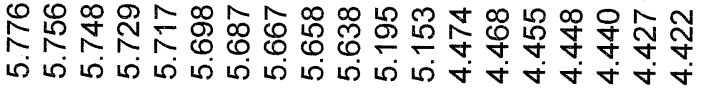

प1 Jل)

||⿲i丨

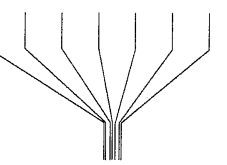

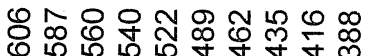

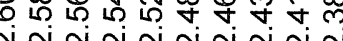

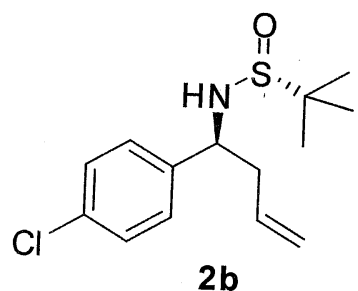

4.23

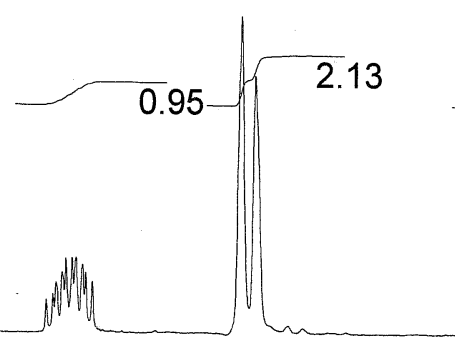

5
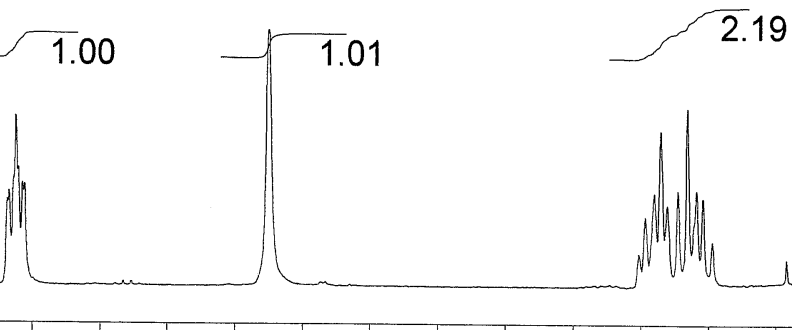


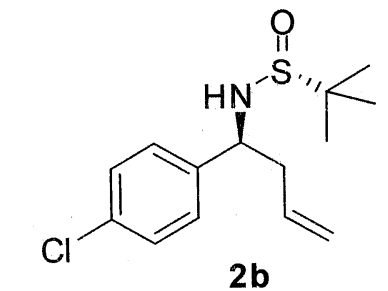

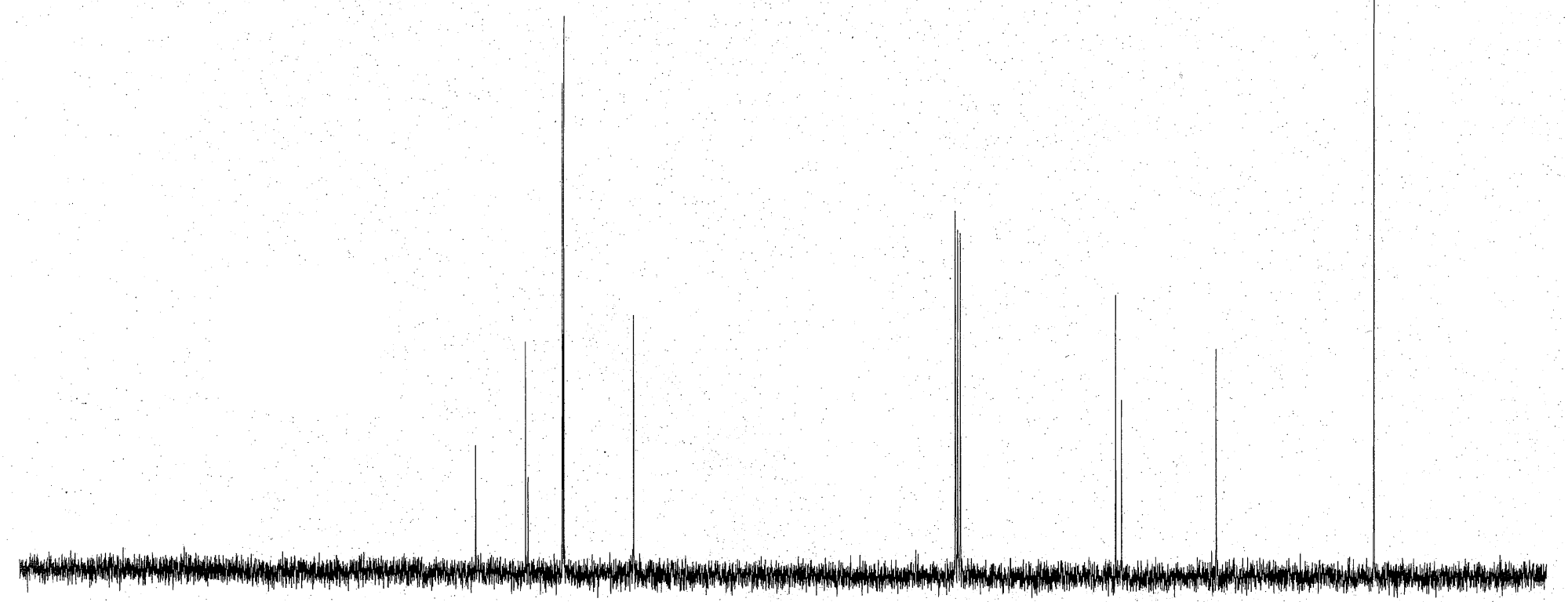

Current Data Parameters NAME.

EXPNO

F2 - Acquisition Parameters

Date_ 20060707

Time-

20060707
4.46

INSTRUM or $\times 400$

PROBHD $5 \mathrm{~mm}$ QNP $13 \mathrm{C}$

PULPROG ZgdC

TD 6553

$\begin{array}{lr}\text { SOLVENT } & \text { DMSO } \\ \text { NS } & 40\end{array}$

SWH $\quad 35211.270 \mathrm{~Hz}$

FIDRES $\quad 0.537281 \mathrm{~Hz}$

$A Q \quad 0.9306612$

$\begin{array}{lr}\text { RG } & 4096 \\ \text { DW } & 14.200 \text { use }\end{array}$

6.00 use

$300.0 \mathrm{~K}$

2. $000000000 \mathrm{sec}$

d11 $0.03000000 \mathrm{sec}$

$=========-$ CHANNEL $f 1$

NUC1 $13 C$

$\begin{array}{ll}\text { P1 } & 6.00 \text { use } \\ \text { PL1 } & -6.00 \mathrm{~dB}\end{array}$

SF01 $100.6224600 \mathrm{MHz}$

$======== \pm=$ CHANNEL f $f$.

CPEPRG2 waltzig

Nic?

waltz16:
$1 \mathrm{H}$

PCPD2 85.00 use

$\begin{array}{ll}P L 2 & 120.00 \mathrm{~dB}\end{array}$

$400.1316020 \mathrm{~dB}$

F2 - Processing parameters

SI $\quad 32768$

WEW $\quad 100.6127723$

SSB $\quad 0$

$\begin{array}{lr}\text { LB } & 1.00 \\ G B & 0\end{array}$

1D. NMR plot parameters

cX $\quad 20.00 \mathrm{~cm}$

$\begin{array}{ll}\text { Fip } & 200.000 \mathrm{ppm} \\ \text { F1 } & 2012255 \mathrm{mp}\end{array}$

$\begin{array}{lr}\text { F1 } & 20122.55 \mathrm{~Hz} \\ \text { F2P } & 0.000 \mathrm{ppm}\end{array}$

F2 $\quad 0.00 \mathrm{~Hz}$

PPMCM $\quad 10,00000 \mathrm{ppm} / \mathrm{cm}$ 

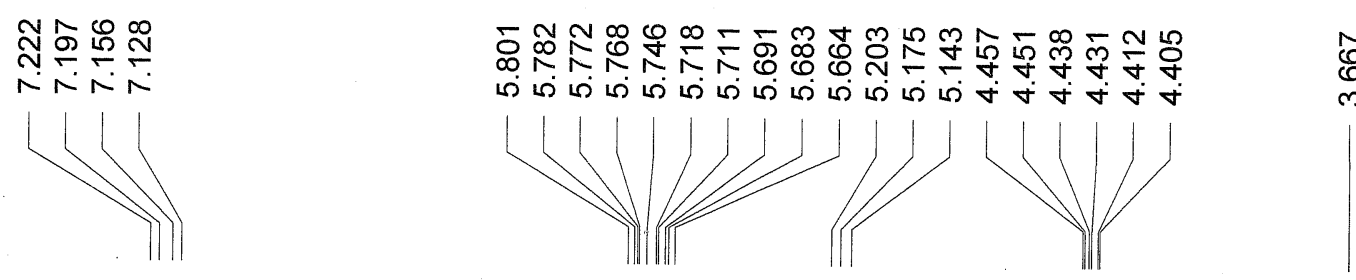

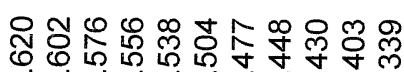

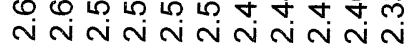
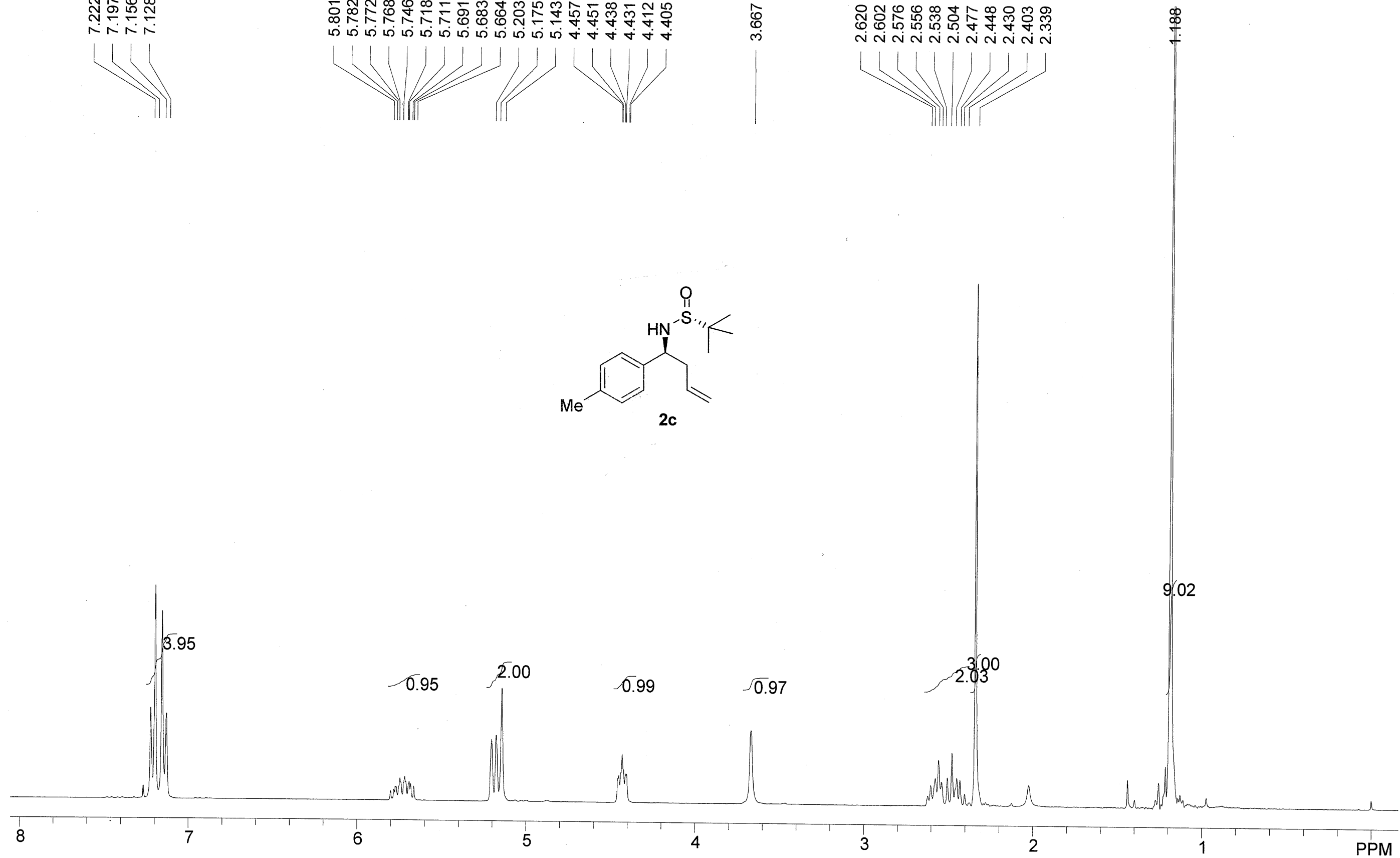

2c 


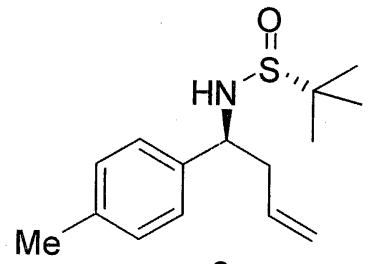

2c

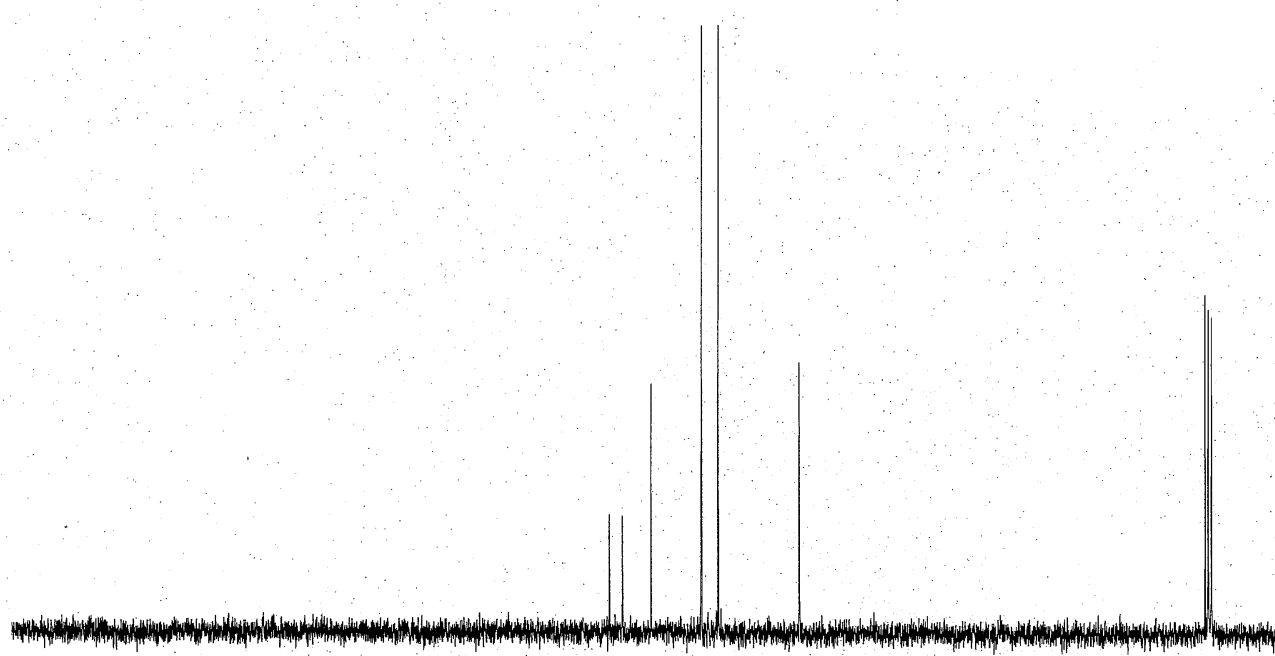

Current Data Parameters NAME EXPNO

C2 - Acquisition Paranters Date__ 20060630 $\begin{array}{lr}\text { Tíme } & 6.49 \\ \text { INSTRUM } & d r \times 400\end{array}$

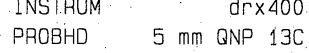
PULLPROG Zgdc TD 65536 $\begin{array}{lr}\text { SOLVENT } & \mathrm{MeOH} \\ \text { NS } & 44\end{array}$ SWH $\quad 35211.270 \mathrm{~Hz}$ $\begin{array}{r}\text { SWHDRES } \quad 0.537281 \mathrm{~Hz} \\ \hline\end{array}$ AQ $\quad 0.9306612 \mathrm{se}$ $\begin{array}{ll}\text { RG } & 4096 \\ \text { DW } & 14.200 \text { usec }\end{array}$ DE 6.00 use TE $\quad 300.0 \mathrm{~K}$ $\begin{array}{ll}01 & 2.00000000 \mathrm{sec} \\ \text { d11 } & 0.03000000 \mathrm{sec}\end{array}$

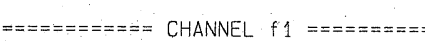
NUC1 $13 \mathrm{C}$ P1 6.00 usec SFO1 $100.6227290 \mathrm{MHZ}$ $==========$ CHANEL fo CPDPAG2 waltz16. $\begin{array}{ll}\text { PCPDC } & \text { 85.00 usec }\end{array}$ PL2 $\quad 120.00 \mathrm{~dB}$

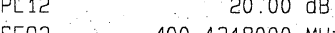

52 - Processing paraters

SI $\quad 32768$

SF. $100.6127726 \mathrm{MHz}$

WDW
SSB

LB $\quad 1.00 \mathrm{~Hz}$

$\begin{array}{lr}\text { GB } & 0 \\ \text { PC } & 0.80\end{array}$

1D NMR plot parameters

CX $\quad 20.00 \mathrm{~cm}$

F1P 200.000 ppm

$\begin{array}{lr}F 1 & 20122.55 \mathrm{~Hz} \\ F 2 & 0.000 \mathrm{ppm} \\ \text { F2P } & 0.00 \mathrm{~Hz}\end{array}$

$\begin{array}{ll}\text { F2P } & 0.000 \mathrm{ppm} \\ \text { F2 } & 0.00 \mathrm{~Hz}\end{array}$

PPMCM $\quad 10.00000 \mathrm{ppm} / \mathrm{cm}$ 


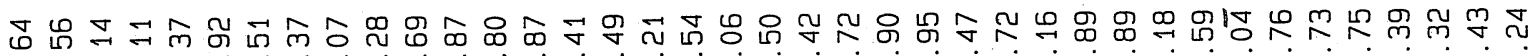

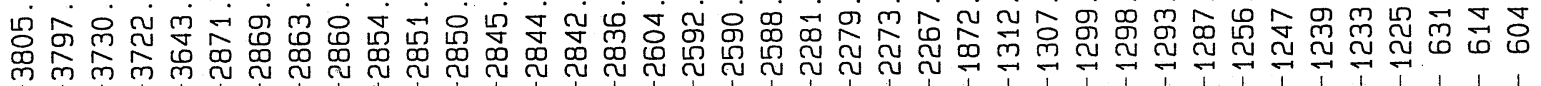
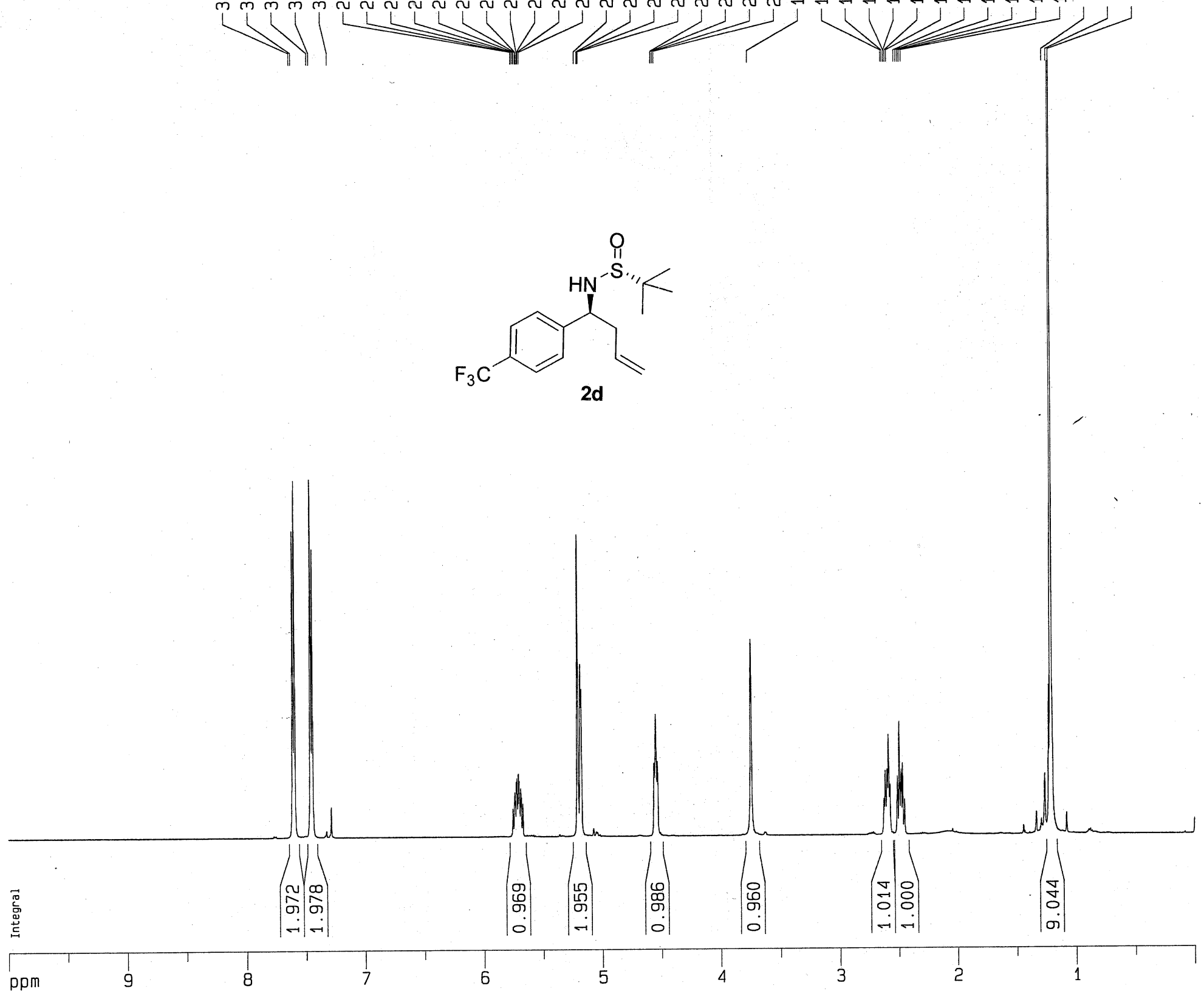

Current Data Parameters

NAME

EXPNO

2 - Acquisition Parameters

Date_ 20061207

INSTRUM av500

PROBHD $5 \mathrm{~mm}$ TXO 19F/1

29
PULPROG

SOLVENT DMSO

SWH $\quad 15015.015 \mathrm{~Hz}$

FIDRES $\quad 0.229111 \mathrm{~Hz}$

AQ 2.1824322 sec

$\begin{array}{lc}\text { AG } & 64 \\ \text { DW } & 33.300 \text { usec }\end{array}$

DE, $\quad 6.50$ use

$\begin{array}{cc}\text { TE } & 300.0 \mathrm{~K} \\ \mathrm{D} 1 & 1.0000000 \mathrm{sec}\end{array}$

$=======$ CHANNEL $f 1$ =s=s=s=$1 \mathrm{H}$

$\quad 500.1310 .00 \mathrm{~dB}$

F2 - Processing parameters

$\begin{array}{lc}\text { SI } & 65536 \\ \text { SF } & 500.1300003 \mathrm{MHz}\end{array}$

WDW no

LB $\quad 0.00 \mathrm{~Hz}$

$\mathrm{PC}$

0.80

10 NMR plot parameters

CX $\quad 20.00 \mathrm{~cm}$

CY $\quad 0.00 \mathrm{~cm}$

$\begin{array}{lr}\text { F1P } & 10.000 \mathrm{ppm}\end{array}$

F2P $\quad 0.000 \mathrm{pp}$

F2 $\quad 0.00 \mathrm{~Hz}$

$\begin{array}{lr}\text { PPMCM } & 0.50000 \mathrm{ppm} / \mathrm{cm} \\ \text { HZCM } & 250.06500 \mathrm{~Hz} / \mathrm{cm}\end{array}$ 
총 ํํㅇ

도용

응 哭

宫品

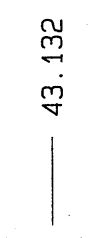
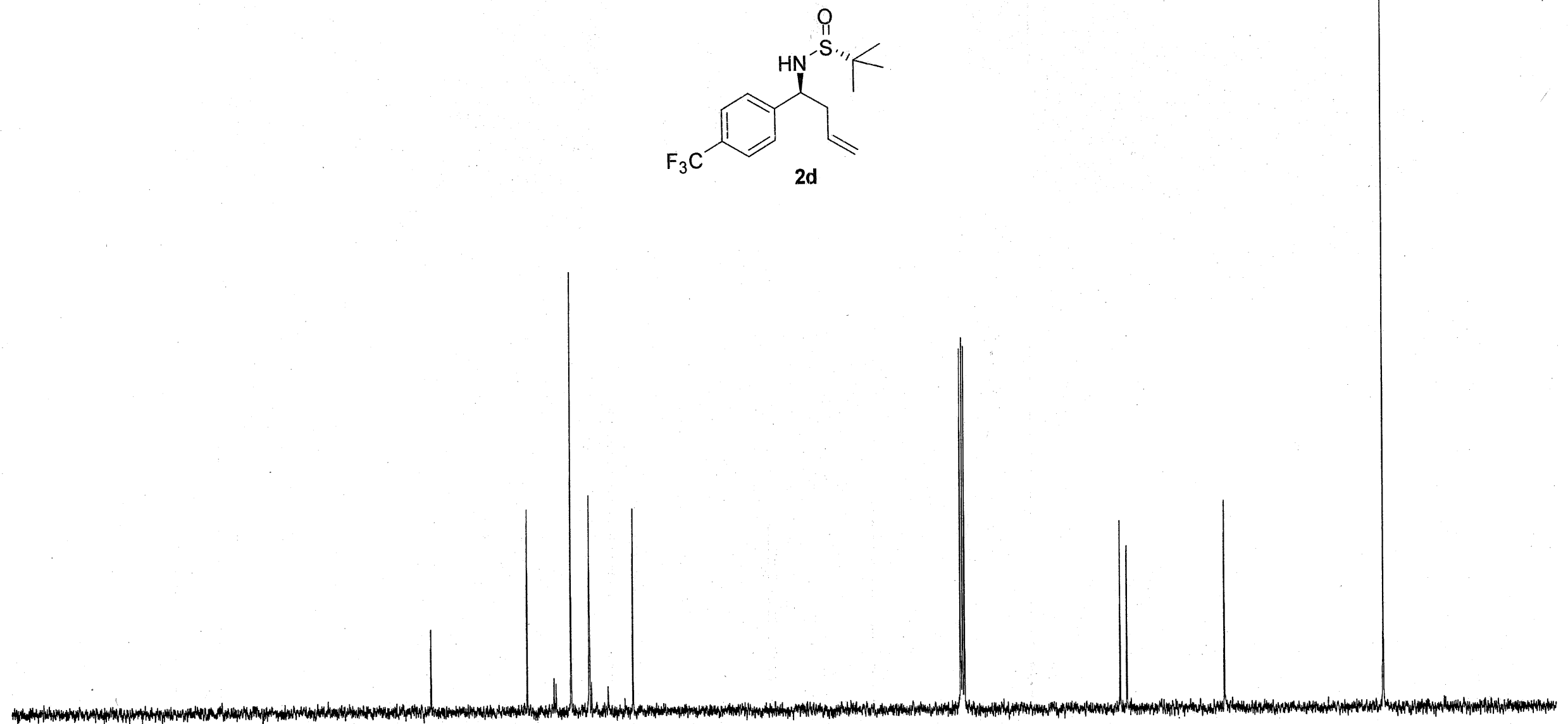


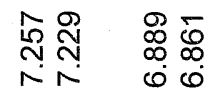

u
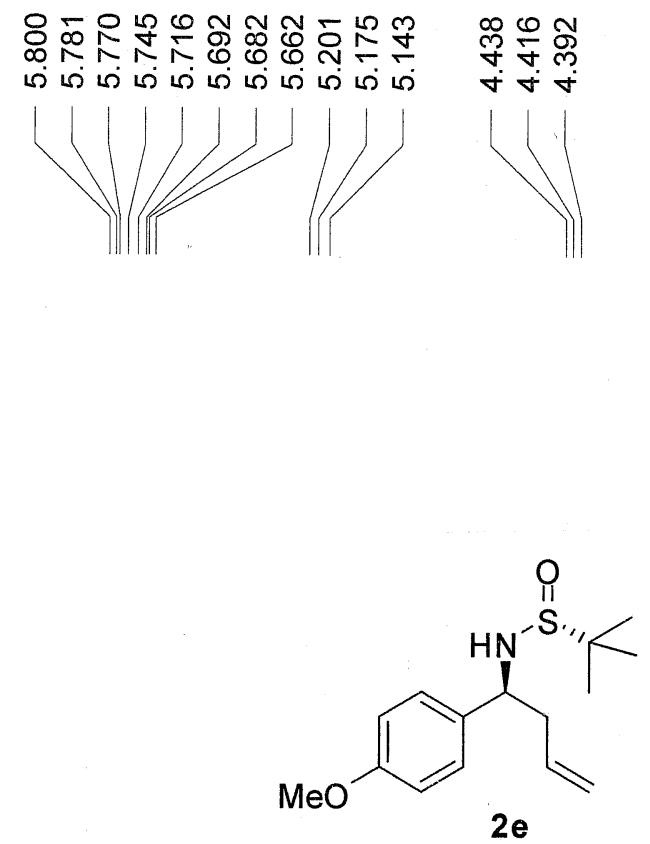

苍

$m$

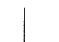

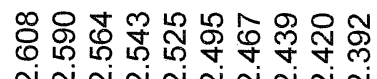

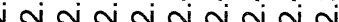

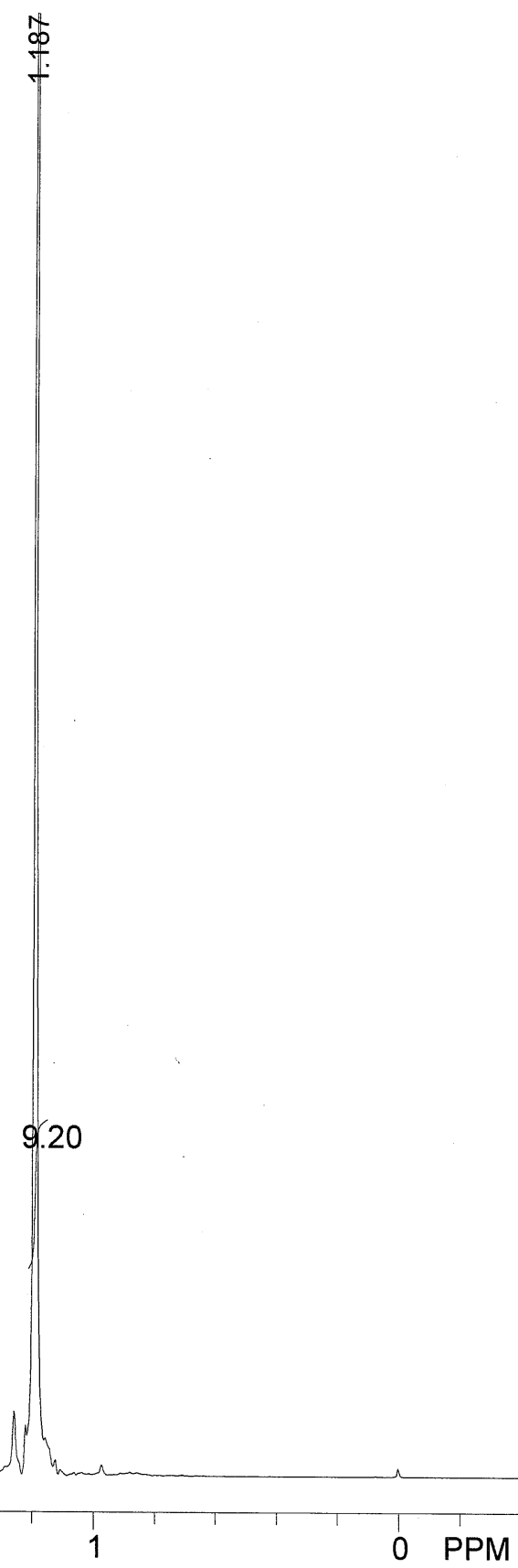




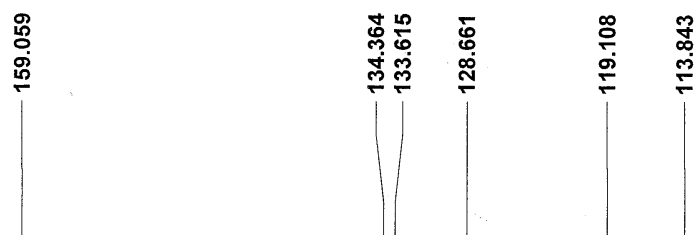

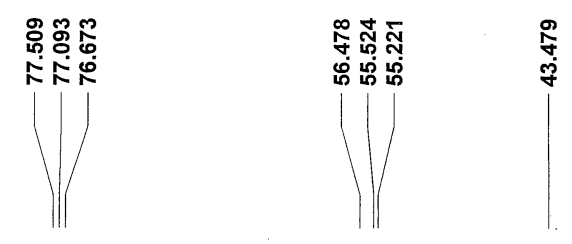
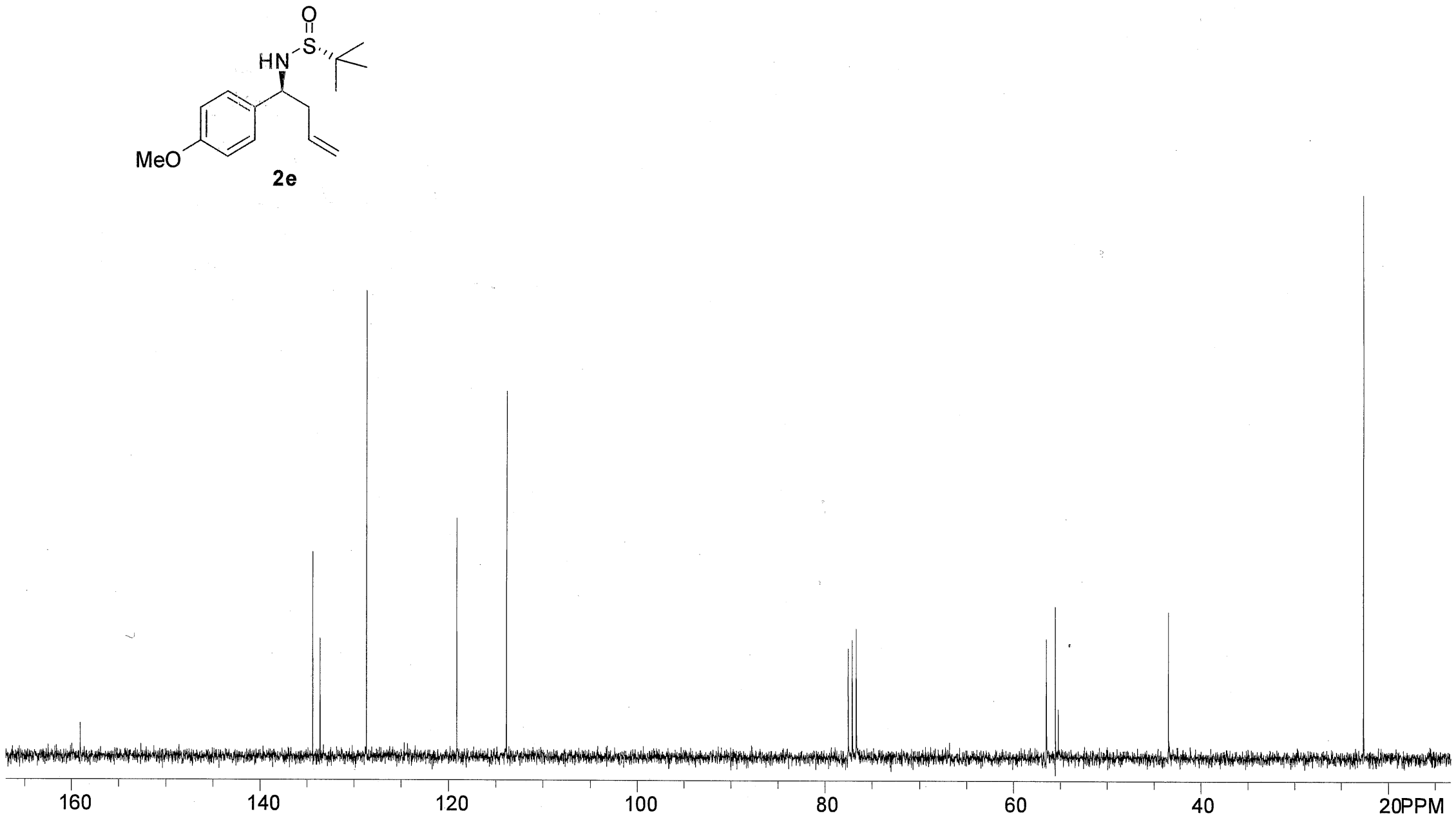


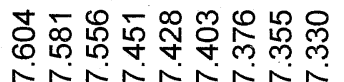
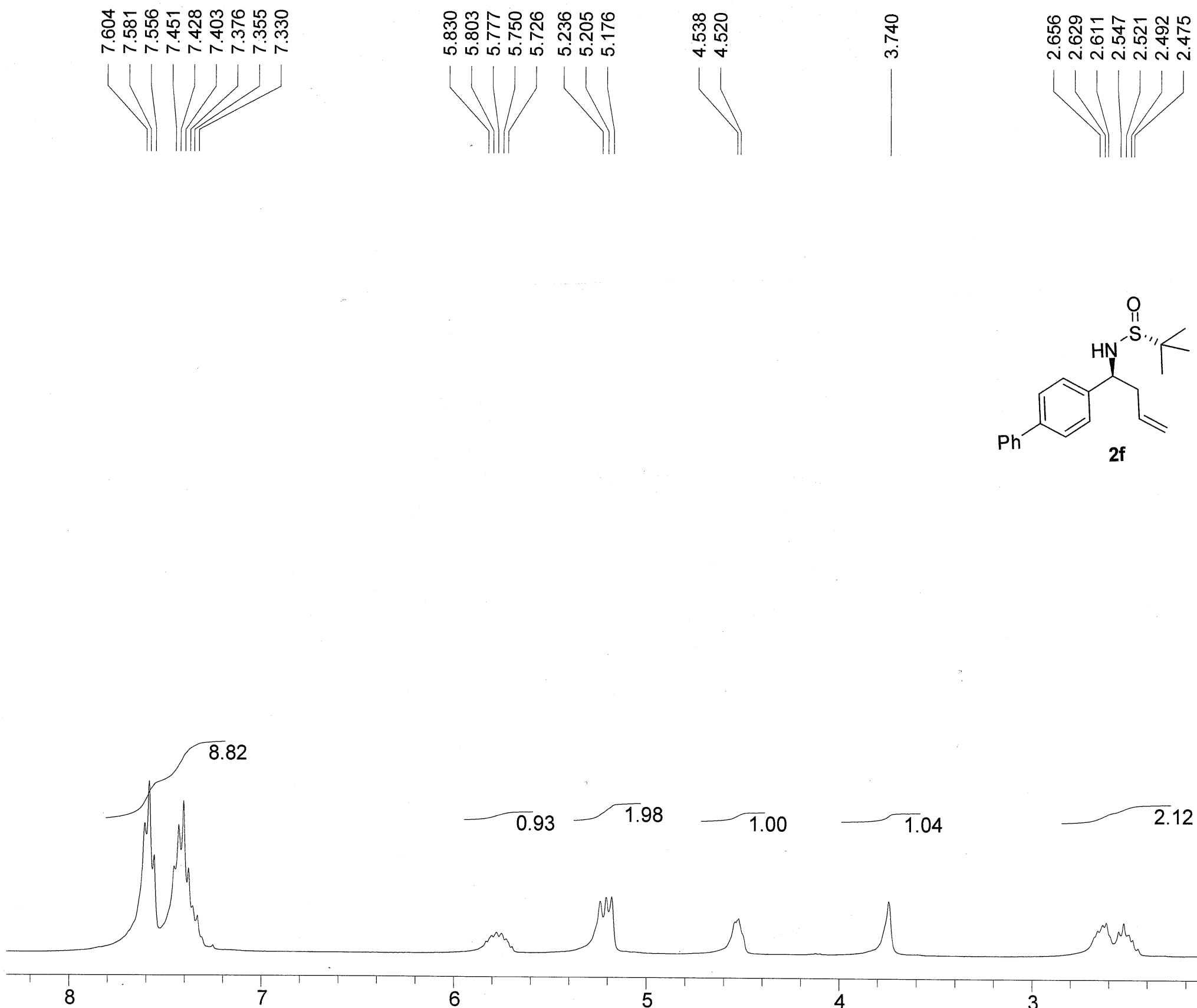

8.82

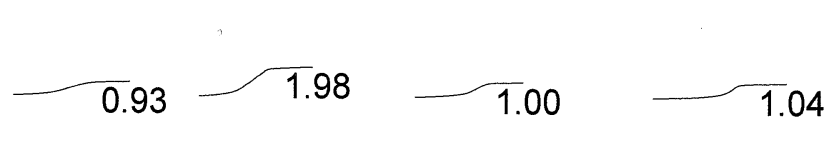

2.12
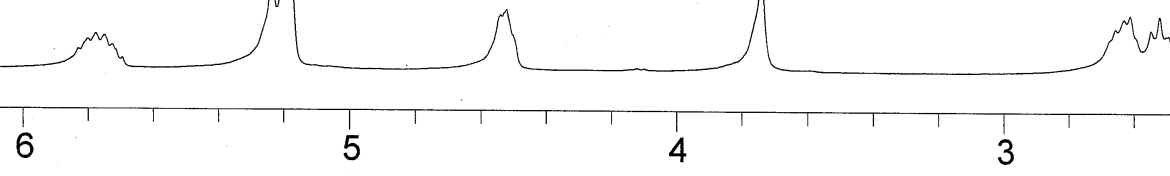

5
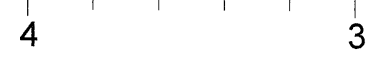


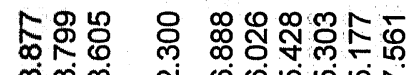

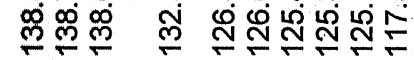

(1)

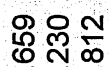

논

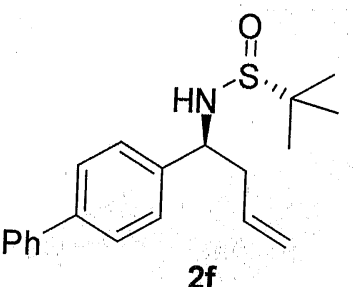

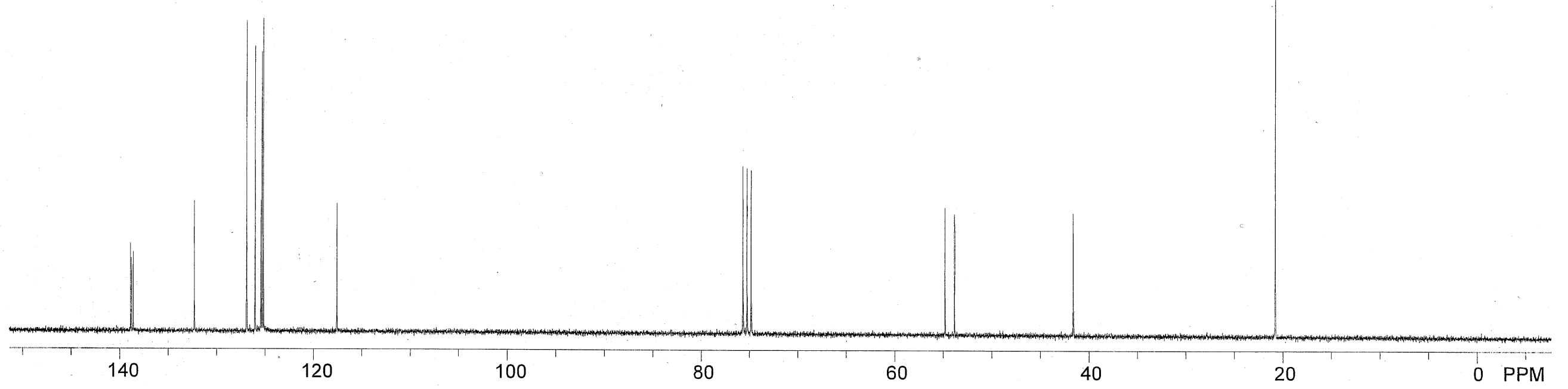



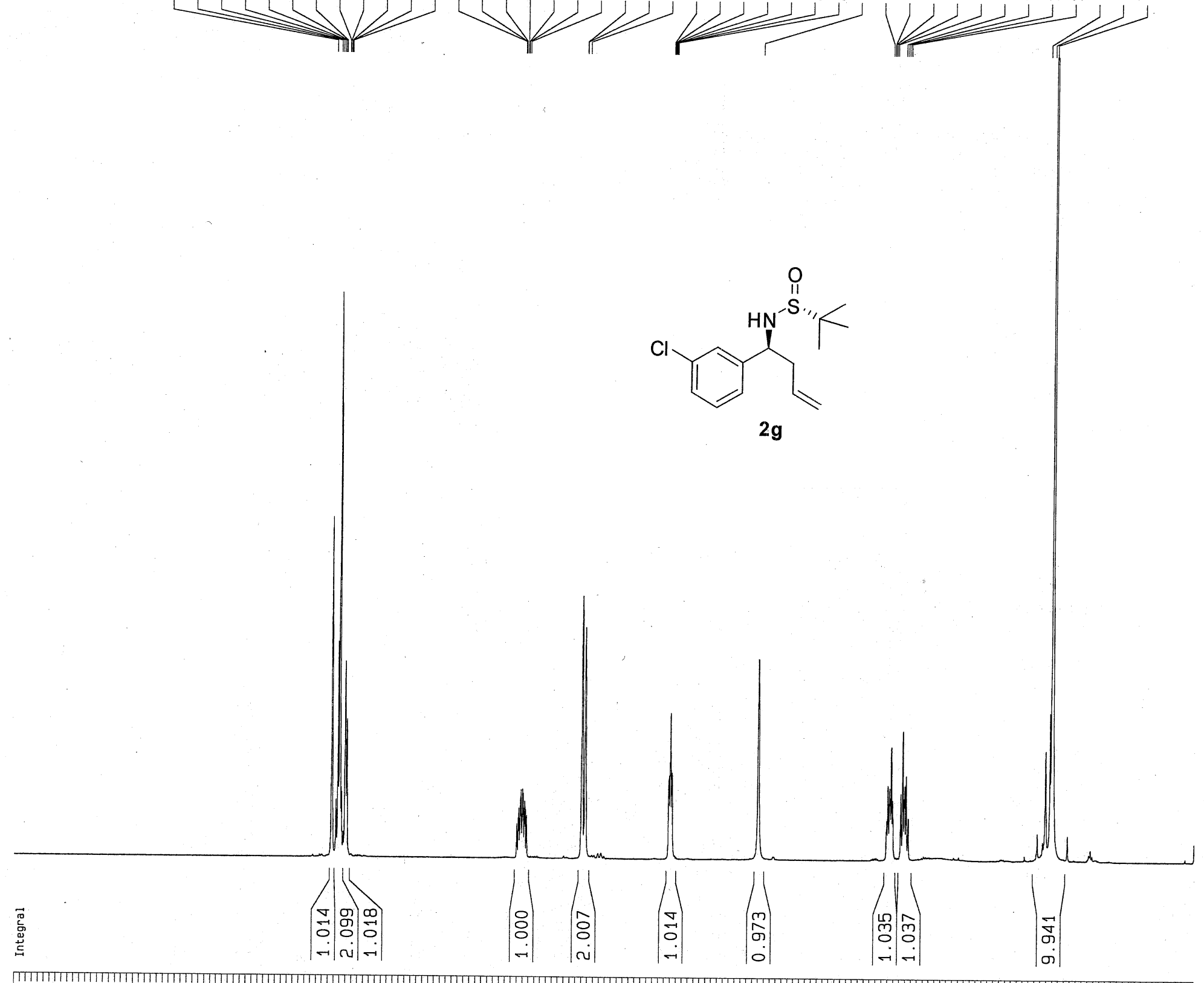

Cürrent Data Parameters

NAME

EXPNO

F2 - Acquisition Parameters

Date_.

20061208

Time

INSTRUM

PROBHD

8.50
5500

TD

SOLVENT $\quad 60056$

$\begin{array}{lr}\text { DS } & 4 \\ \text { SWH } & 15015.015 \mathrm{HZ}\end{array}$

FIDRES $\quad 0.250017 \mathrm{~Hz}$

$A Q \quad 1.9999481 \mathrm{sec}$

$\mathrm{RG}$

DW

DE

TE

33. 64

6.50 usec

$\begin{array}{ll}300.0 \mathrm{~K} \\ \mathrm{D} 1 & 2.00000000 \mathrm{sec}\end{array}$

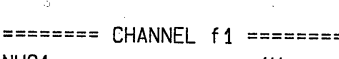

NUC1 $\quad 1$ H

$\begin{array}{ll}\text { P1 } & 4.00 \text { usec } \\ \text { PL1 } & 0.00 \mathrm{~dB}\end{array}$

$\begin{array}{lr}\text { SFO1 } & 0.00 \mathrm{~dB} \\ & 500.1321411 \mathrm{MHz}\end{array}$

F2 - Processing parameters

SI 65536

$\quad 500.1300003 \mathrm{MHz}$

WDW

LB

GB

no

$0.00 \mathrm{~Hz}$

$0.00 \mathrm{~Hz}$
0

10 NMR plot parameters

$\begin{array}{cr}\mathrm{CX} & 20.00 \mathrm{~cm}\end{array}$

$\begin{array}{ll}\text { CY } & 0.00 \mathrm{~cm}\end{array}$

F1 $\quad 5001.30 \mathrm{~Hz}$

F2P $\quad 0.000 \mathrm{~Hz}$

F2 $\quad 0.000 \mathrm{pp}$

$\quad 0.50000 \mathrm{ppm} / \mathrm{cm}$

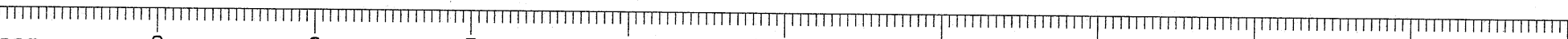




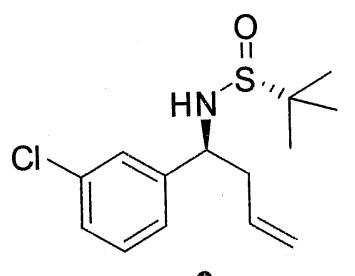

2g

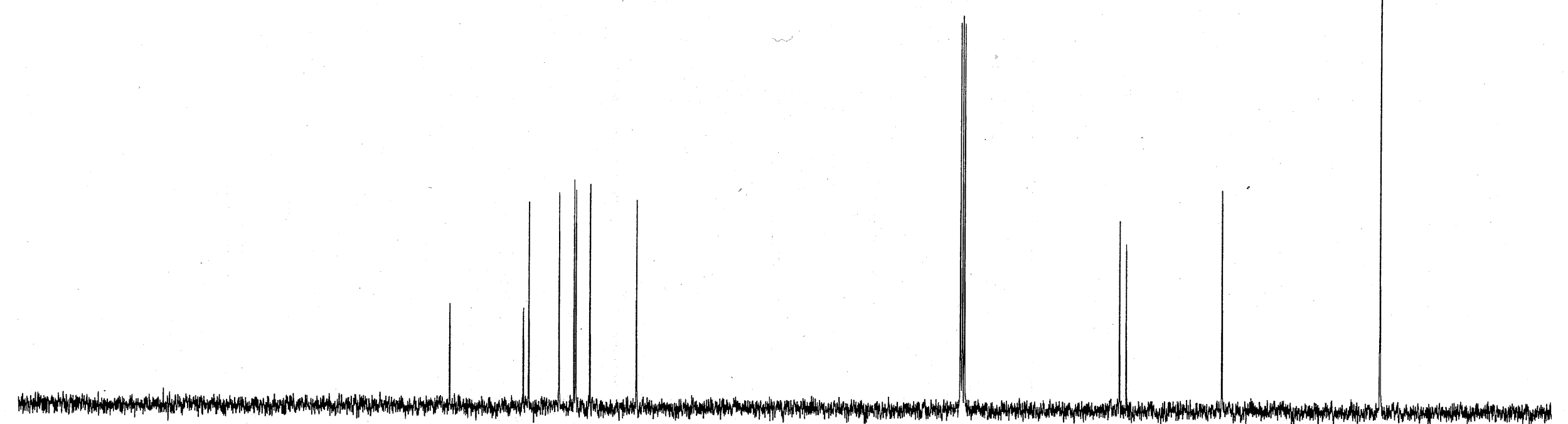




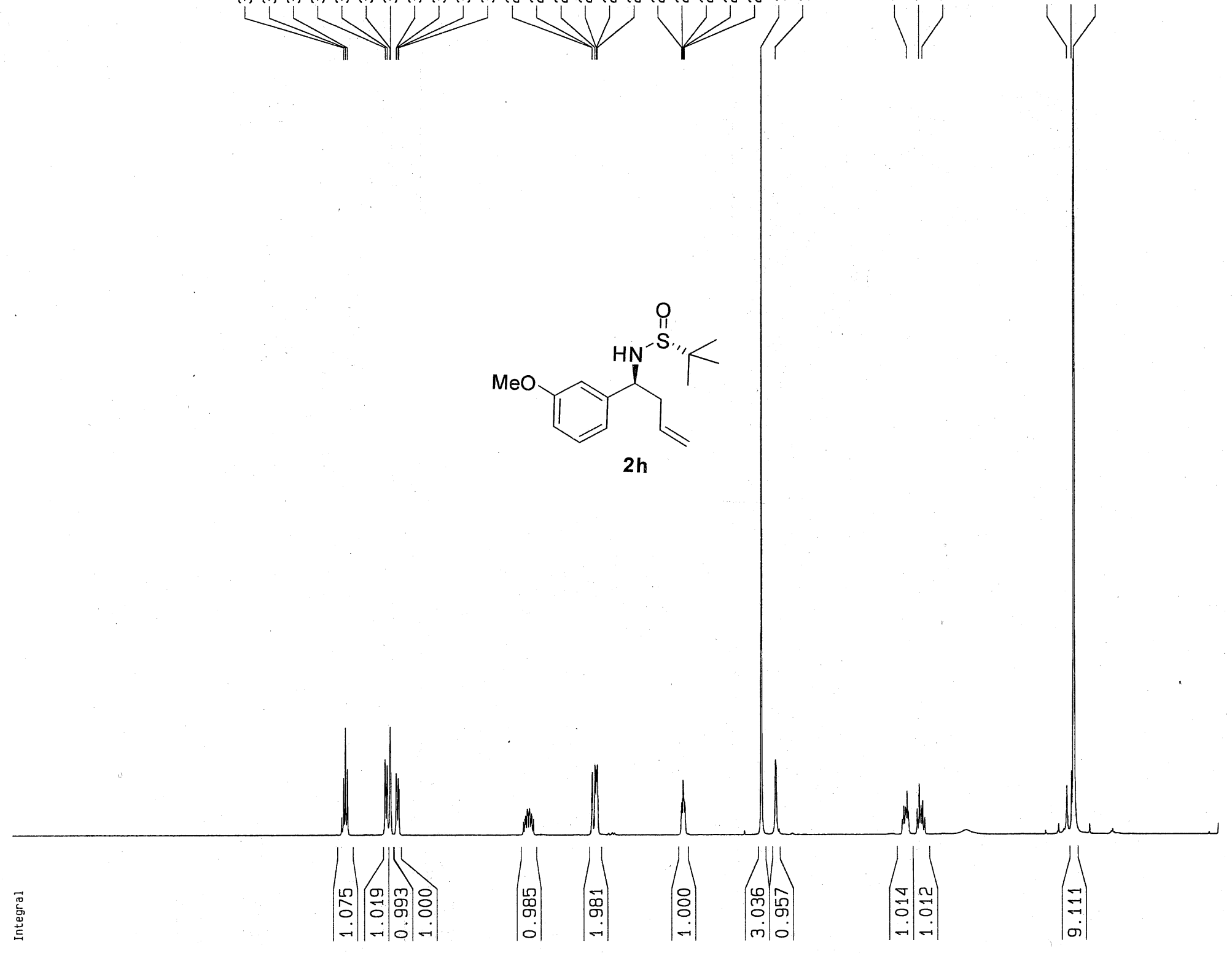

Current Data Parameters NAME EXPNO

PROCNO

F2 - Acquisition Parameters

Date_

Date

20061207

PROBHD $5 \mathrm{~mm}$ TXO 19F/1

PULPROG

TO

SOLVENT

NS
DS

$\begin{array}{lr}\text { DS } & 0 \\ \text { SWH } & 15015.015 \mathrm{~Hz}\end{array}$

IDRES $\quad 0.229111 \mathrm{~Hz}$

2.1824322 se

$D E$

TE

33.300 usec

6.50 use

$300.0 \mathrm{~K}$

$2.00000000 \mathrm{sec}$

$=======$ CHANNEL $+1=======$
NUC1
PH

P1 3.00 usec

PL1 $\quad 0.00 \mathrm{~dB}$

F2 - Processing parameters

$\begin{array}{lc}\text { SI } & 65536 \\ \text { SF } & 500.1300046 \mathrm{MHz}\end{array}$

WDW $\quad 500.130004$

SSB $\quad 0$

$\begin{array}{lr}\text { LB } & 0.00 \\ \text { GB } & 0\end{array}$

10 NMR plot parameters

$20.00 \mathrm{~cm}$

$0.00 \mathrm{~cm}$
$\quad 10.000 \mathrm{pPm}$

$10.000 \mathrm{ppm}$
$F_{1}$

F2P $\quad 5001.30 \mathrm{~Hz}$

F2 $0.000 \mathrm{ppm}$

PPMCM $\quad 0.50000 \mathrm{p} \mathrm{pm} / \mathrm{cm}$

$\mathrm{HZCM} \quad 250.06500 \mathrm{~Hz} / \mathrm{cm}$ 


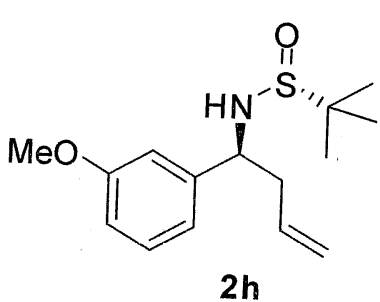

$2 \mathrm{~h}$ 

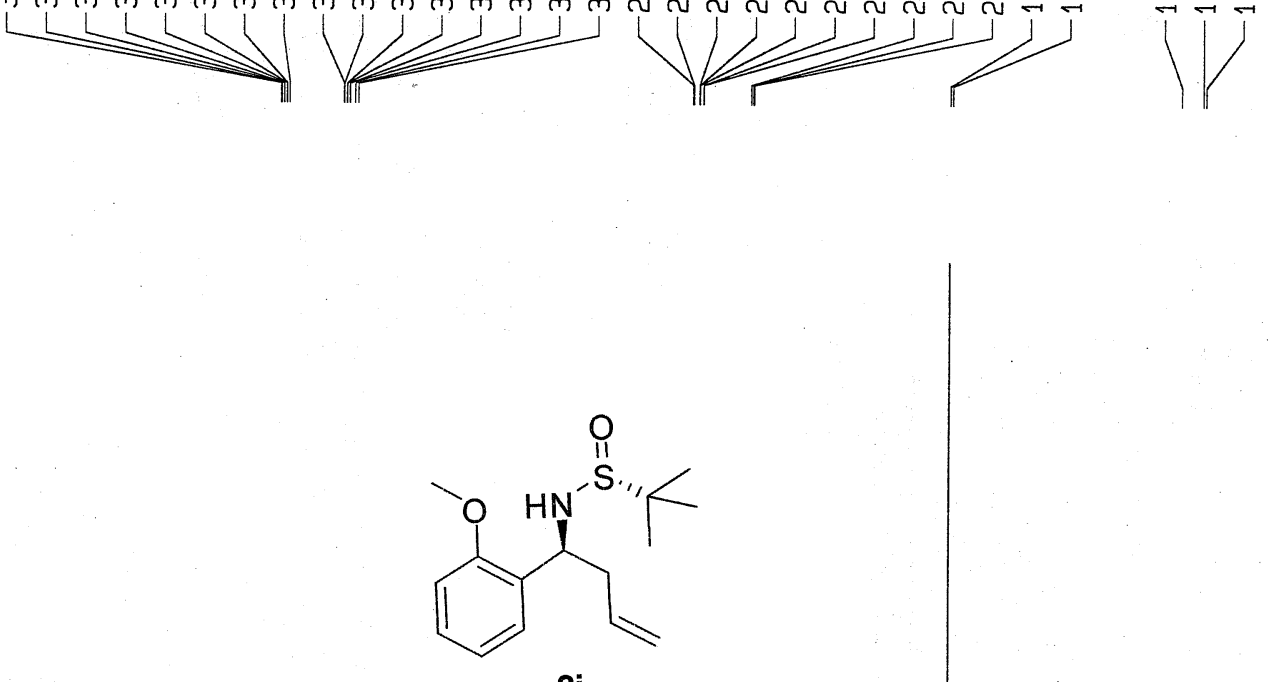

$2 \mathbf{i}$
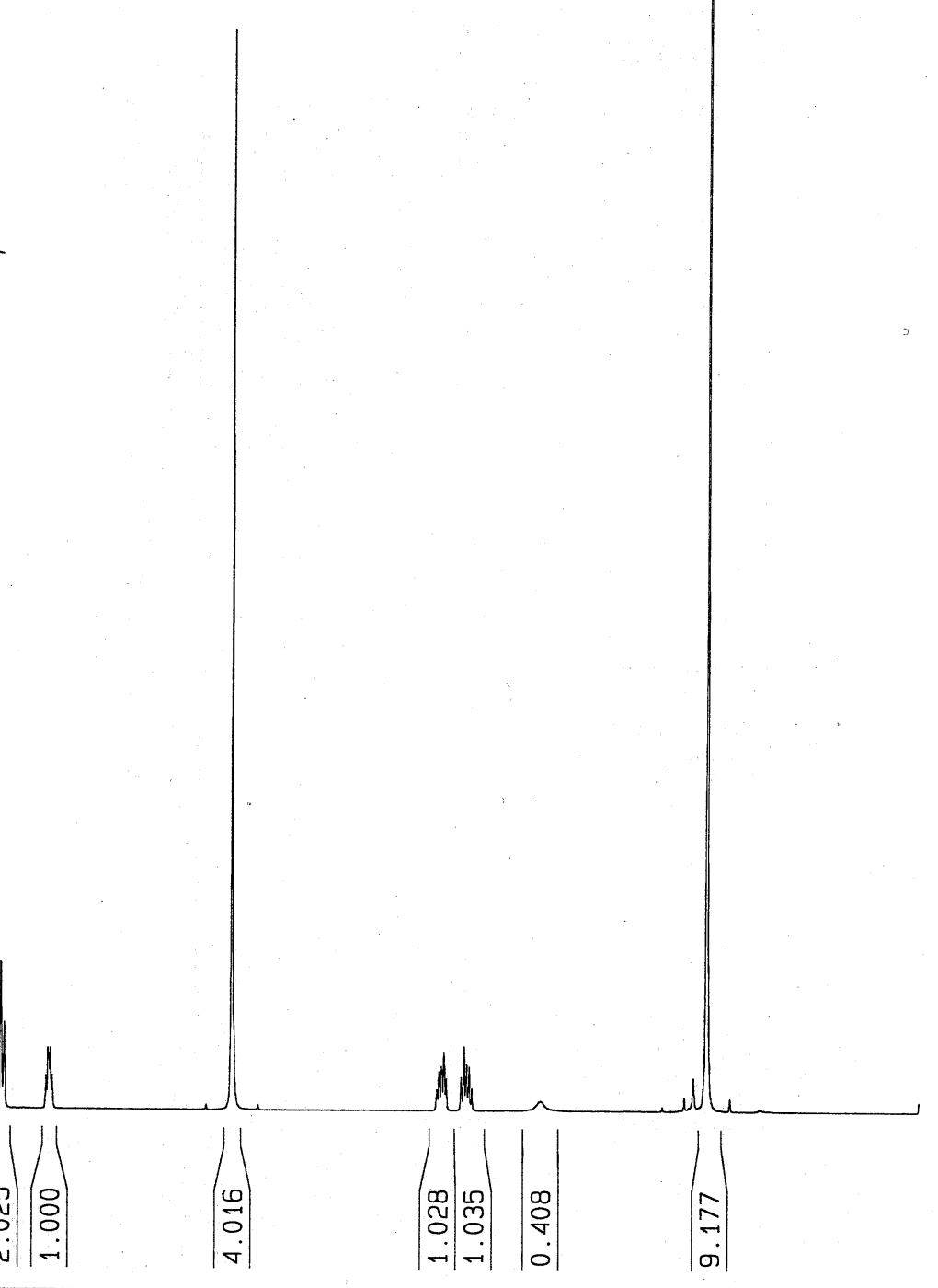

Current Data Parameters

NAME

EXPNO

F2 - Acquisition Parameters

ion Parameter

$\begin{array}{lr}\text { Date_ } & 20061208 \\ \text { Time } & 8.57\end{array}$

$\begin{array}{lr}8.57 \\ \text { INSTRUM } & \text { av500 }\end{array}$

PROBHO $5 \mathrm{~mm}$ TXO $19 \mathrm{~F} / 1$

PULPROG $\quad 29$

TD

60056

NS

$\begin{array}{lc}\text { DS } & 4 \\ \text { SWH } & 15015.015 \mathrm{~Hz}\end{array}$

$15015.015 \mathrm{~Hz}$
FIDRES
$0.250017 \mathrm{~Hz}$

AQ $\quad 1.9999481 \mathrm{sec}$

AQ

DW

$D W$
DE
TE

64

33.300 usec
6.50 usec

$300.0 \mathrm{~K}$

D1 $2.00000000 \mathrm{sec}$

$===== \pm=$ CHANNEL $f 1======$
NUC1

$\begin{array}{lc}\text { NUC1 } & 1 \mathrm{H} \\ \text { P1 } & 4.00 \text { usec }\end{array}$

PL1 $\quad 0.00 \mathrm{~dB}$

500.1321411 MHz

F2 - Processing parameters

SI 65536

SF $\quad 500.1300060 \mathrm{MHz}$

WDW

LB

GB

no
0

$0.00 \mathrm{~Hz}$

0

10 NMR plot parameters

$\begin{array}{ll}\mathrm{CX} & 20.00 \mathrm{~cm}\end{array}$

$\begin{array}{lr}\text { CY } & 0.00 \mathrm{~cm}\end{array}$

$\begin{array}{lr}F_{1} & 10.000 \mathrm{ppm}\end{array}$

F2P $\quad 5001.30 \mathrm{~Hz}$

F2 $\quad 0.000 \mathrm{ppm}$

PPMCM $\quad 0.50000 \mathrm{ppm} / \mathrm{cm}$

HZCM

$250.06500 \mathrm{~Hz} / \mathrm{cm}$ 
요용

怘哭 芦

추응

岗出芯

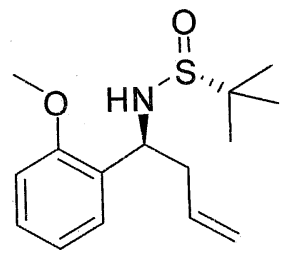

2i 


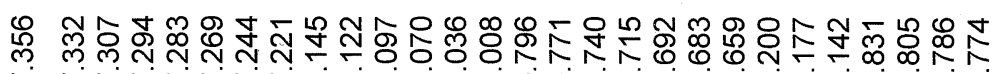

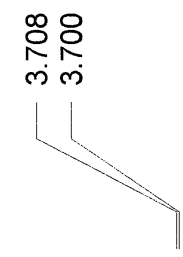

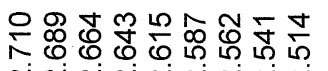

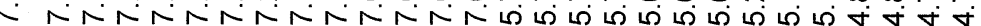

L(L) J)」J]

诸
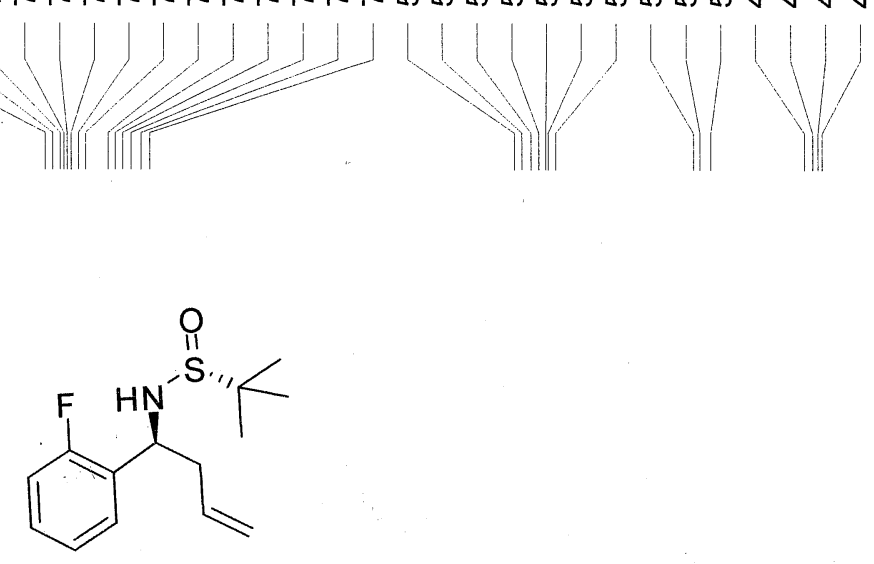

2)

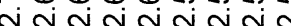

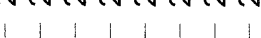

4.05

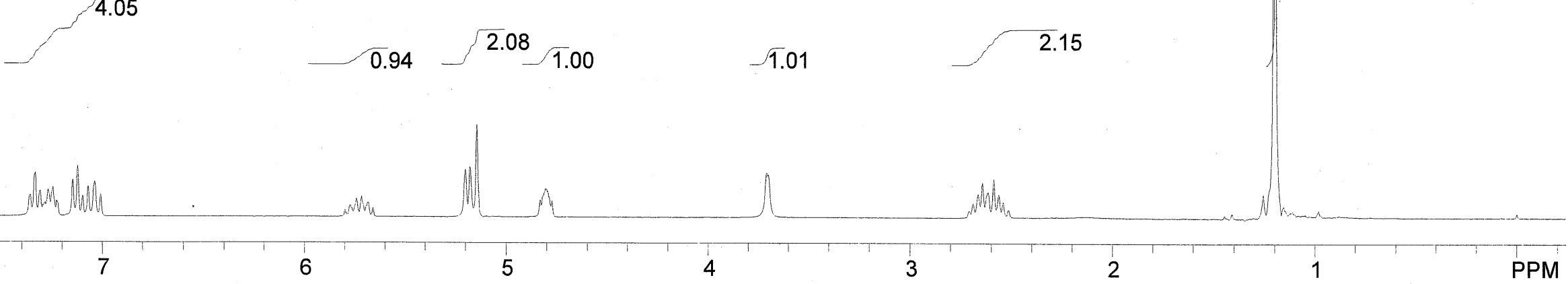




$\mid$
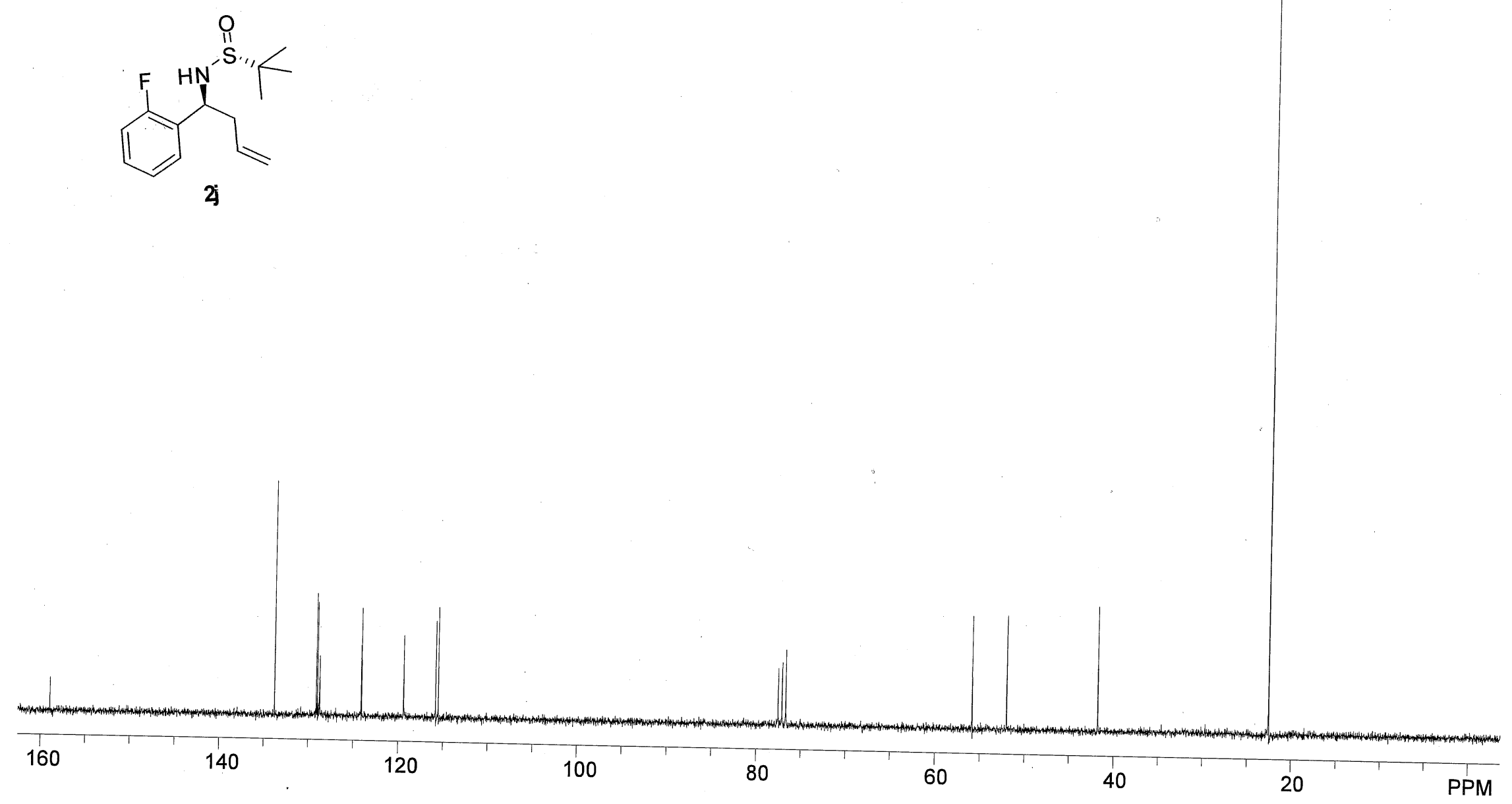


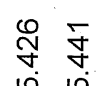

官

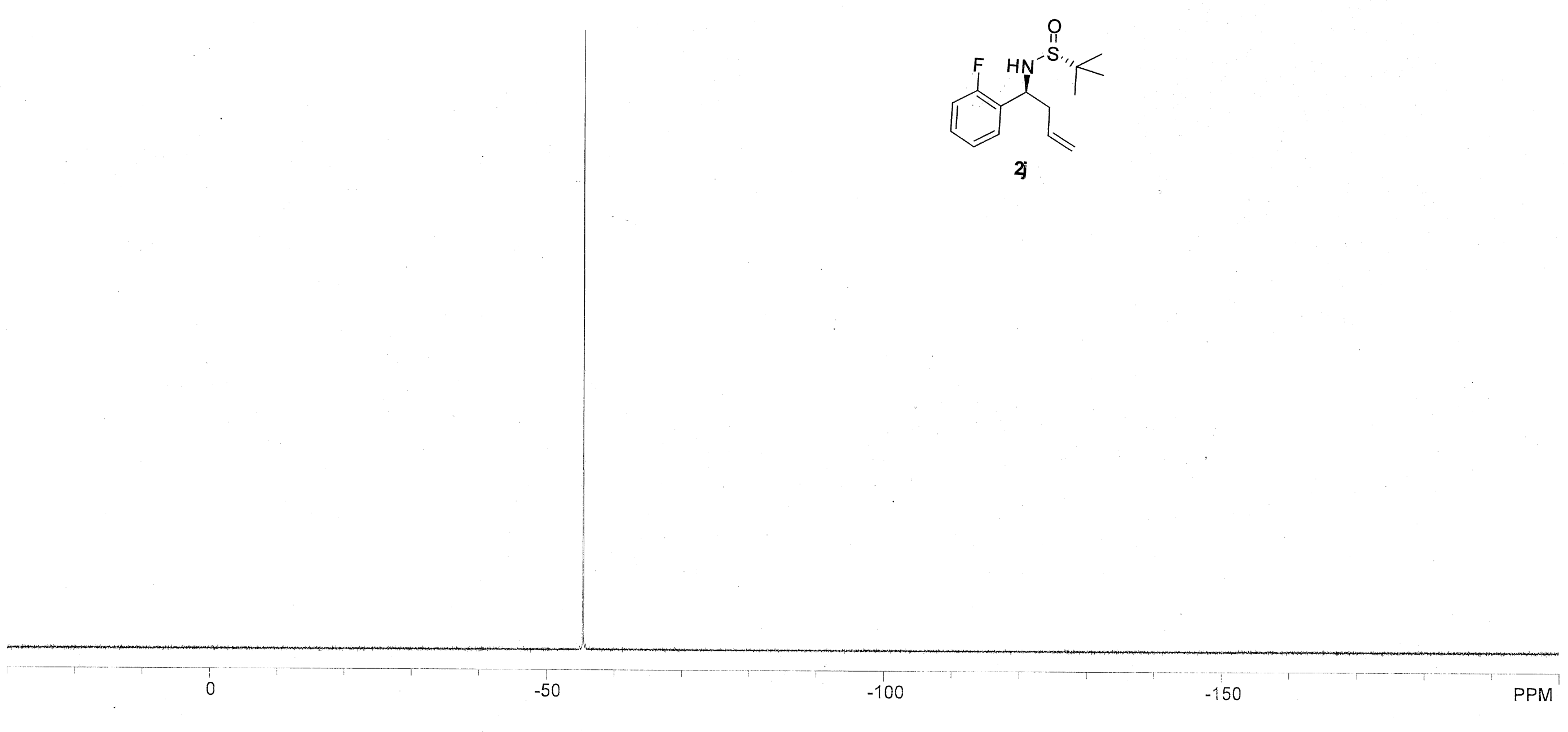




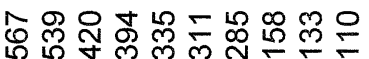
NNNNNN公 L L L L L

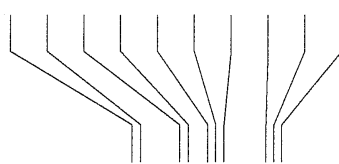

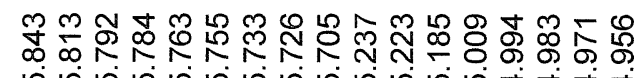

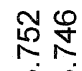

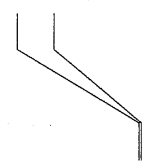

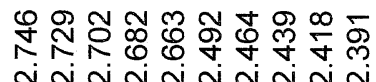

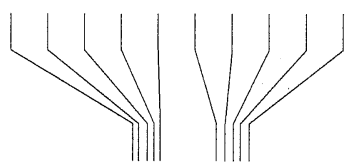

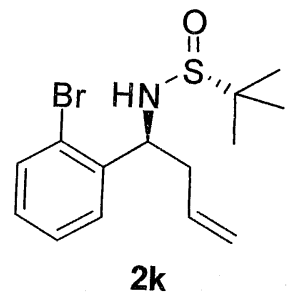
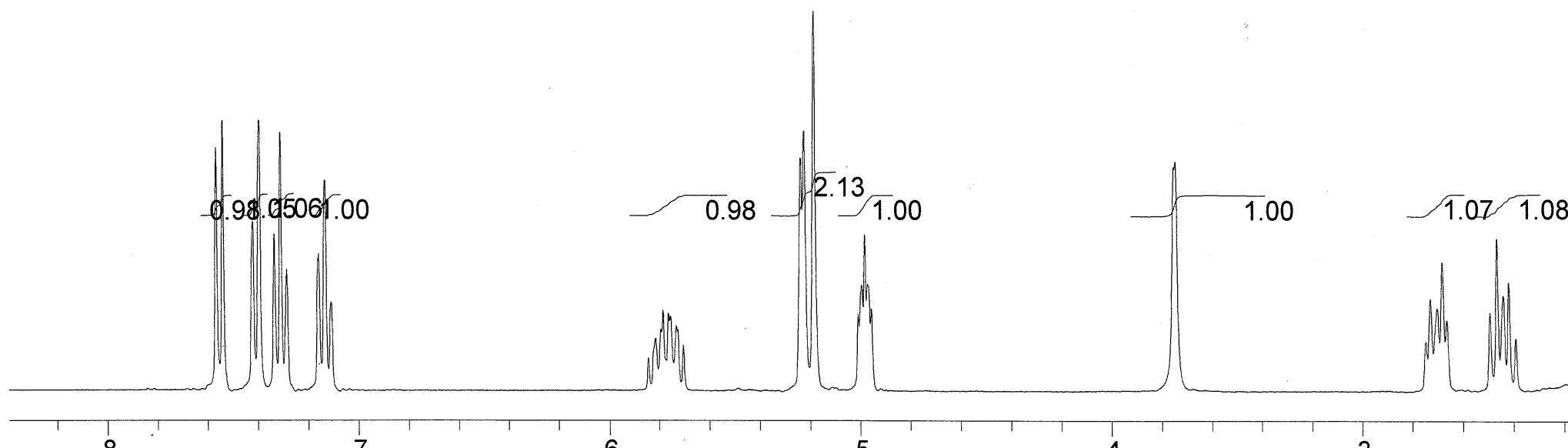


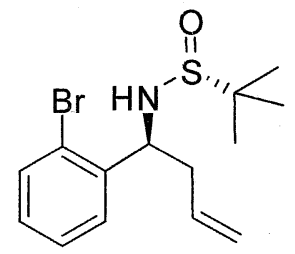

2k

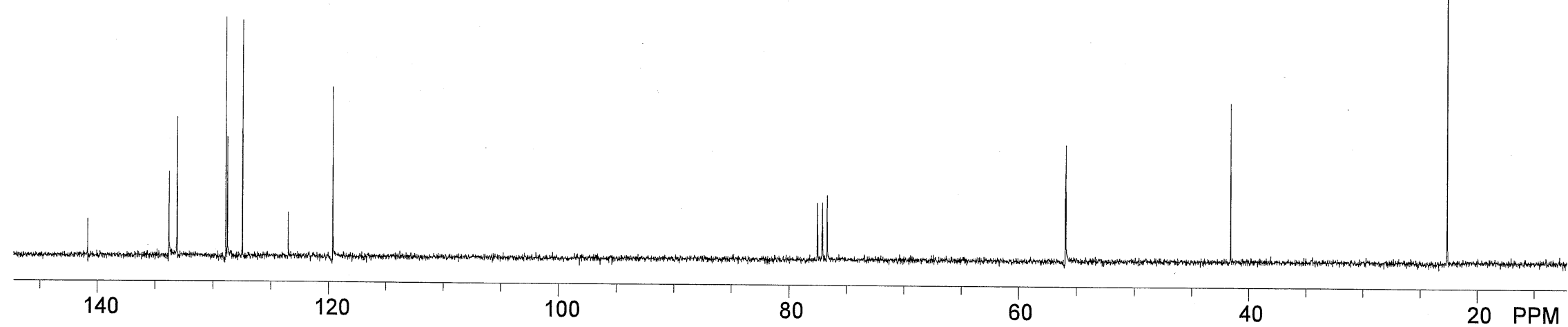



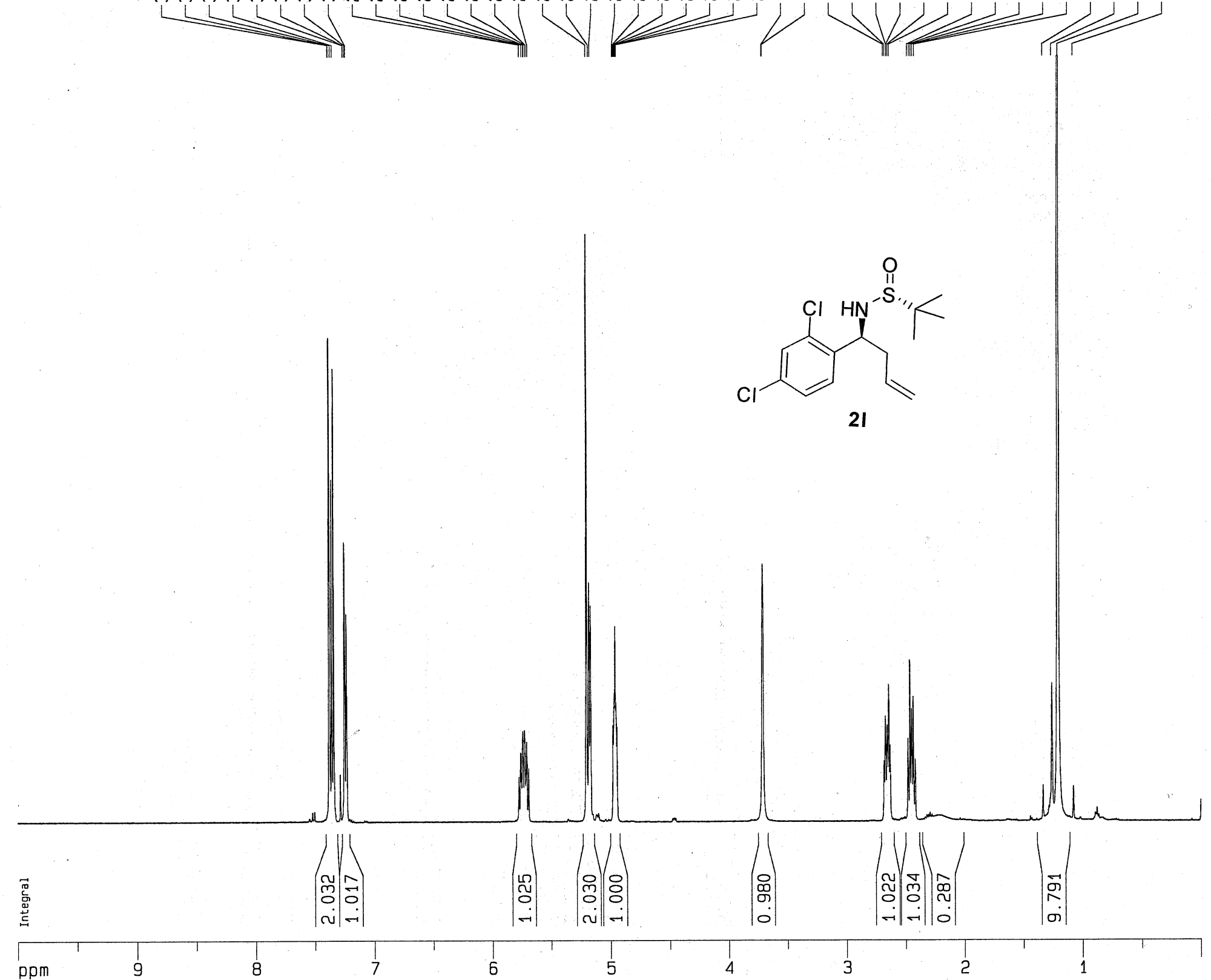

Current Data Parameters

F2 - Acquisition Parameter

Date_ $\quad 20061207$

15.56

INSTRUM

av500

PROBHD $5 \mathrm{~mm}$ TXO 19F/1

PULPROG

TD

SOLVENT

4

0
SWH
$15015.015 \mathrm{~Hz}$

FIDRES $\quad 0.229111 \mathrm{~Hz}$

AQ $\quad 2.1824322 \mathrm{sec}$

AG 64

$\begin{array}{lr}\text { DW } & 33.300 \text { usec } \\ \text { DE } & 6.50 \text { usec }\end{array}$

$300.0 \mathrm{~K}$

$2.00000000 \mathrm{sec}$

$\begin{array}{lr}=======\text { CHANNEL } f 1 & ======= \\ \text { NUC1 } & 1 \mathrm{H} \\ \text { P1 } & 4.00 \mathrm{useC} \\ \text { PL1 } & 0.00 \mathrm{~dB} \\ \text { SFO1 } & 500.1320806 \mathrm{MHz}\end{array}$

$\quad 0.00 \mathrm{~dB}$

F2 - Processing parameters

SI $\quad 65536$

SF

WDW

LB

no

$0.00 \mathrm{~Hz}$

0

10 NMR plot parameters

CX $20.00 \mathrm{~cm}$

$\begin{array}{lr}\mathrm{CY} & 0.00 \mathrm{~cm}\end{array}$

$\begin{array}{lr}\mathrm{Fr}_{1} & 10.000 \mathrm{ppm} \\ F_{1} & 5001.30 \mathrm{~Hz}\end{array}$

F2P $\quad 0.000 \mathrm{pom}$

$20.00 \mathrm{~Hz}$

$\begin{array}{lr}\text { PPMCM } & 0.50000 \mathrm{ppm} / \mathrm{cm} \\ \text { HZCM } & 250.06500 \mathrm{~Hz} / \mathrm{cm}\end{array}$ 
뚱ํㅇ용

총

V
品哭

出㖹

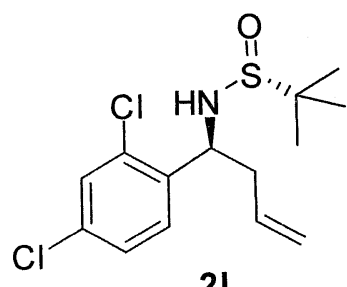

21

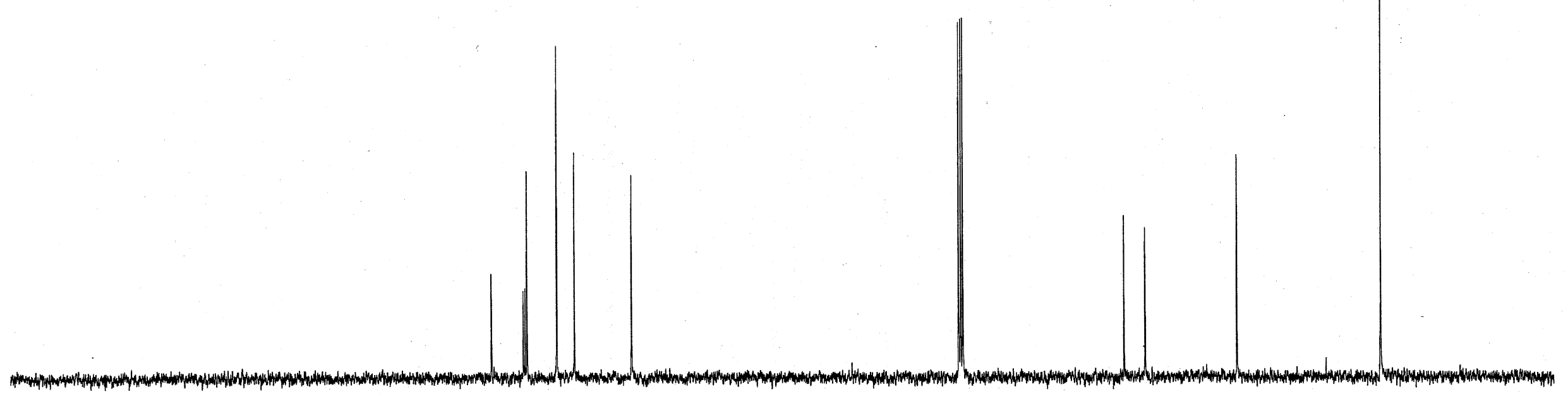



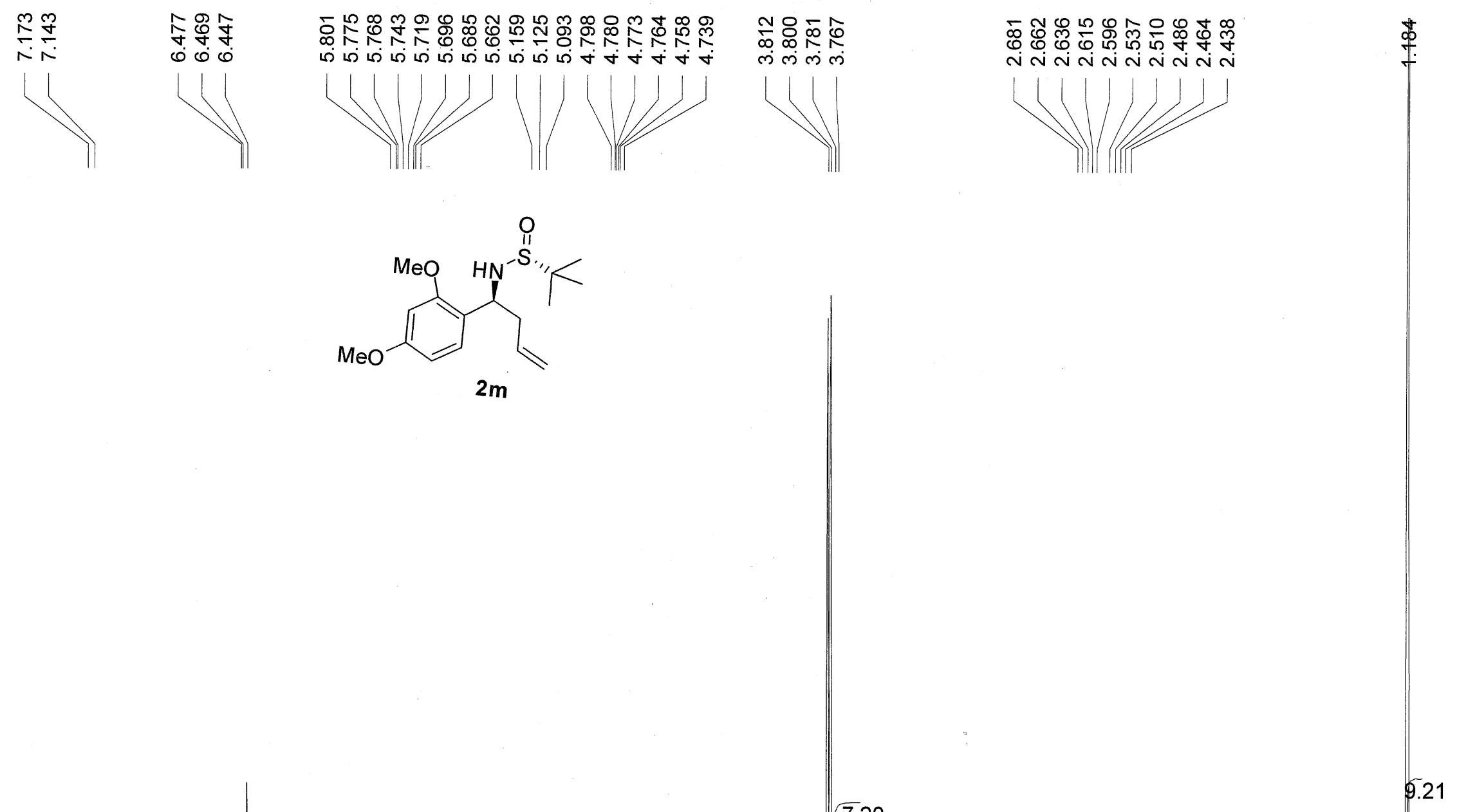

7.20

2.09

N w w

PPM 


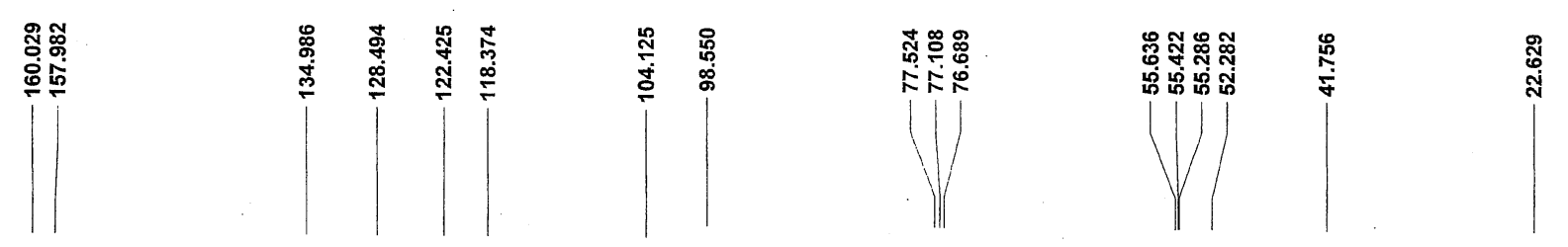
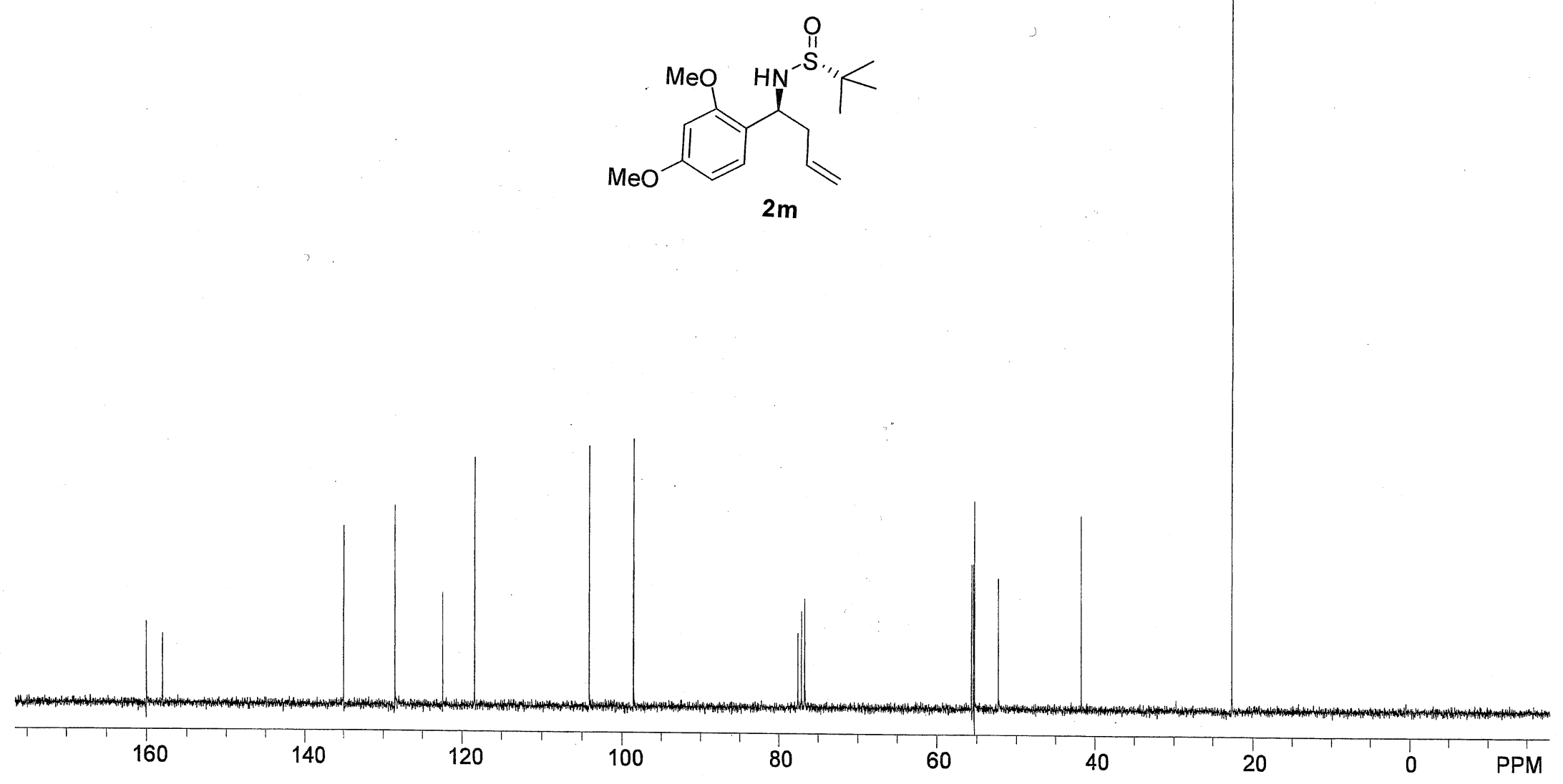

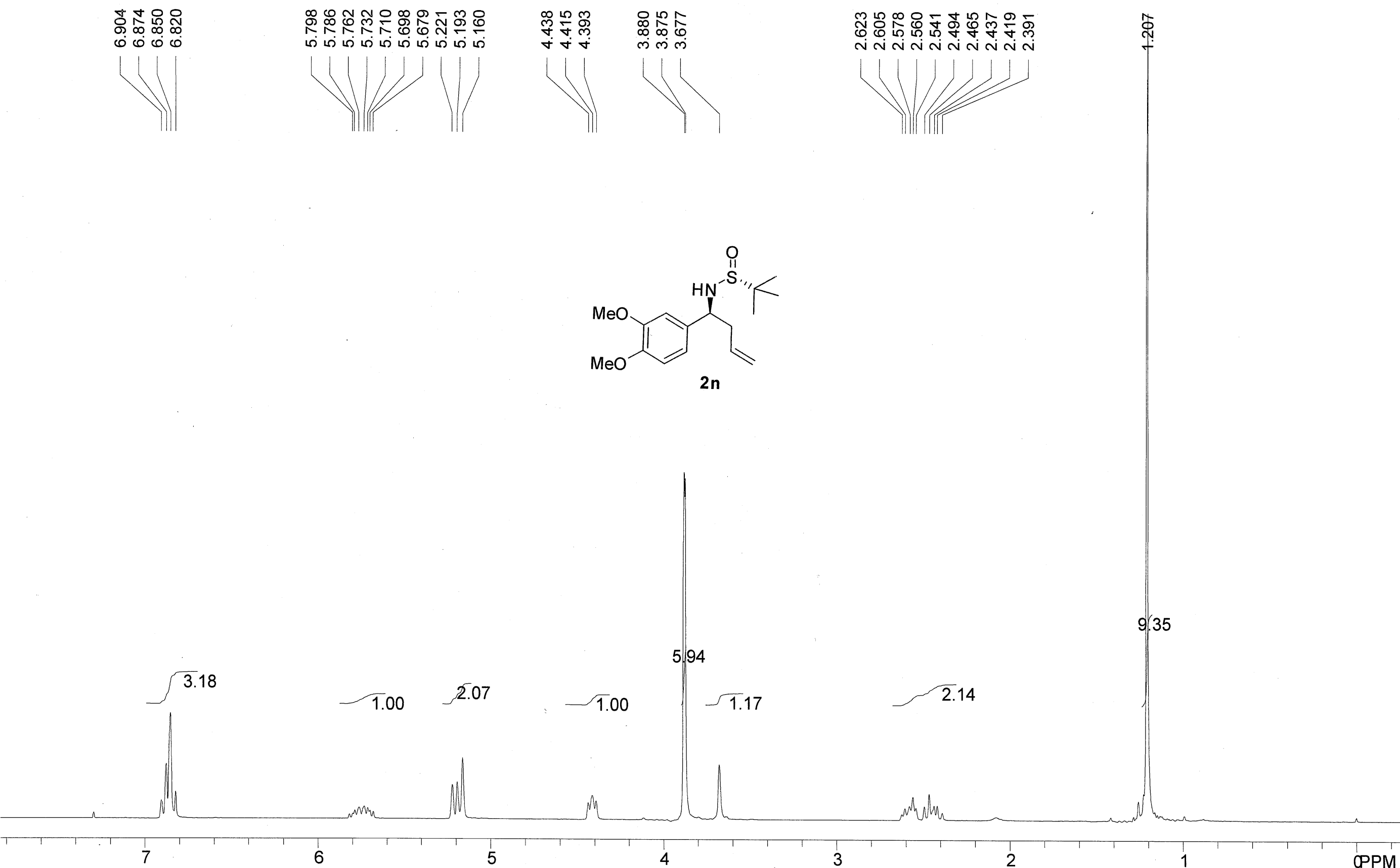


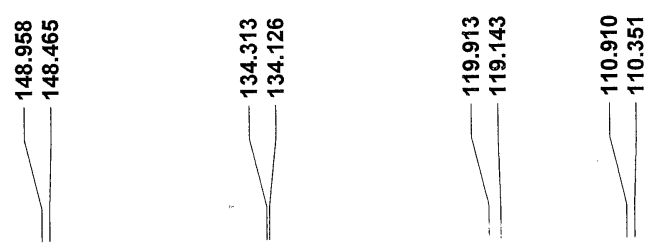

||
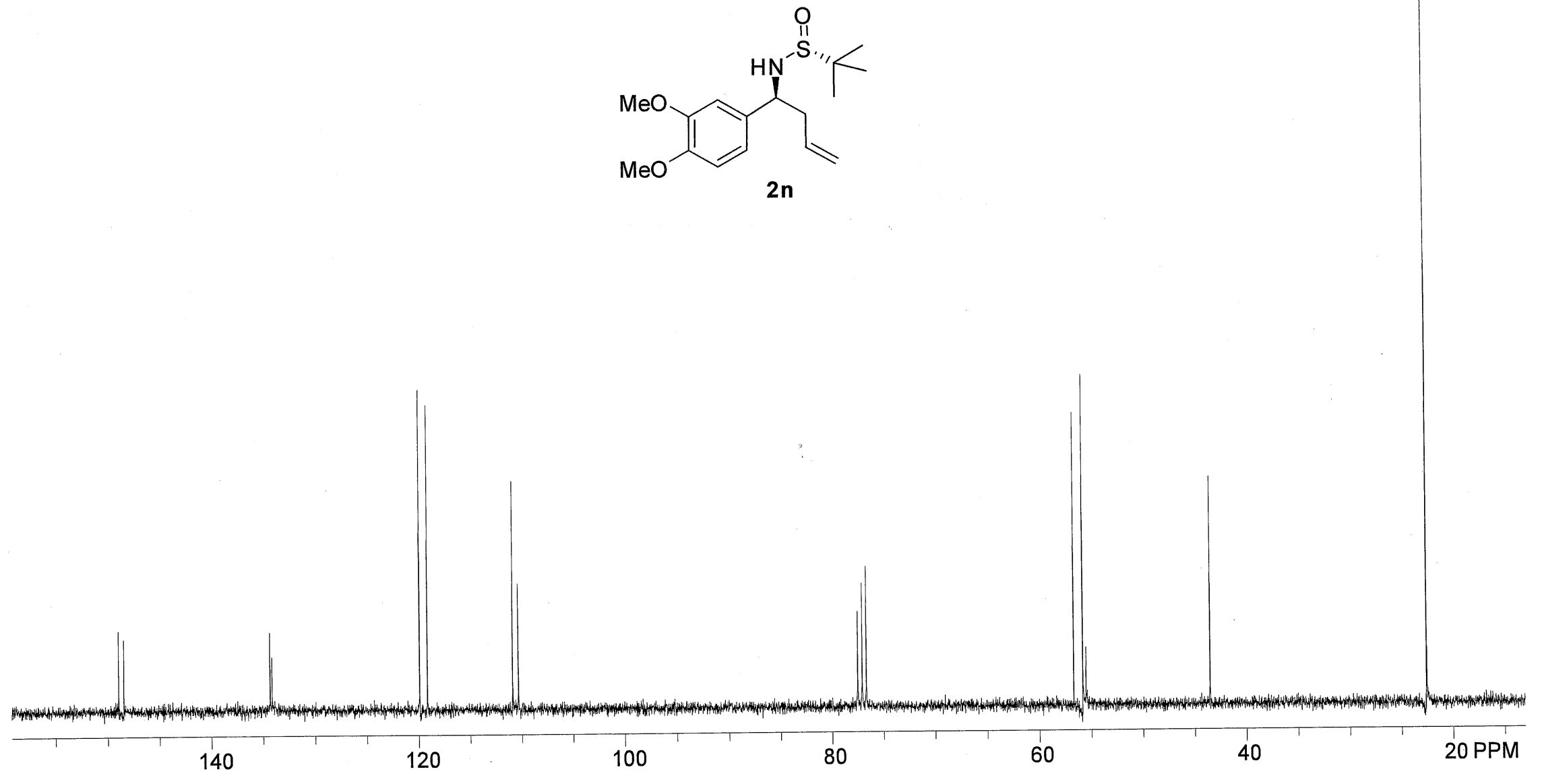


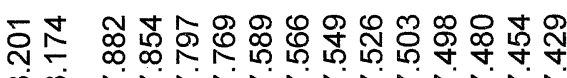

$\infty \infty$ 每

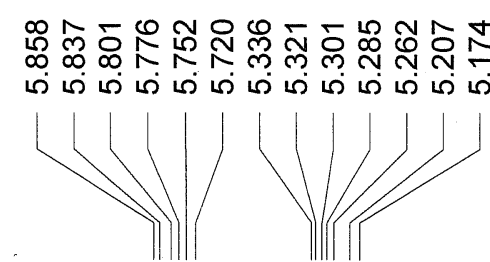

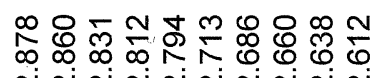

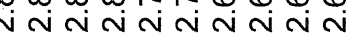

लm

4

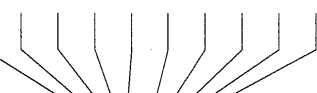

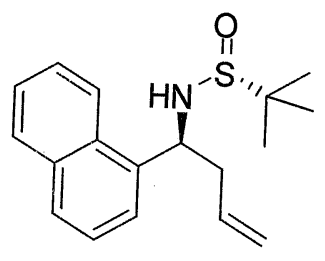

20
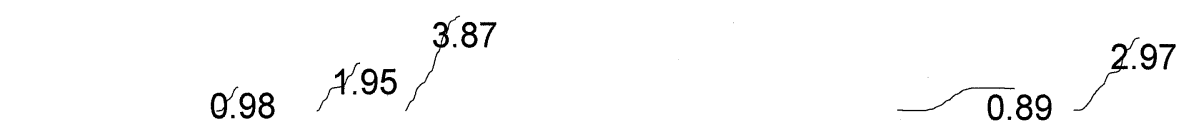

0.92

1.011 .00

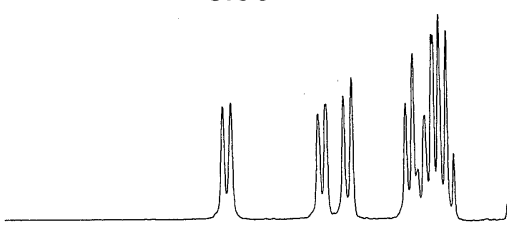

8

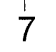

6
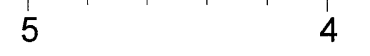

3 


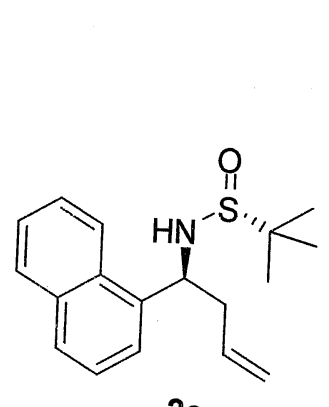

20

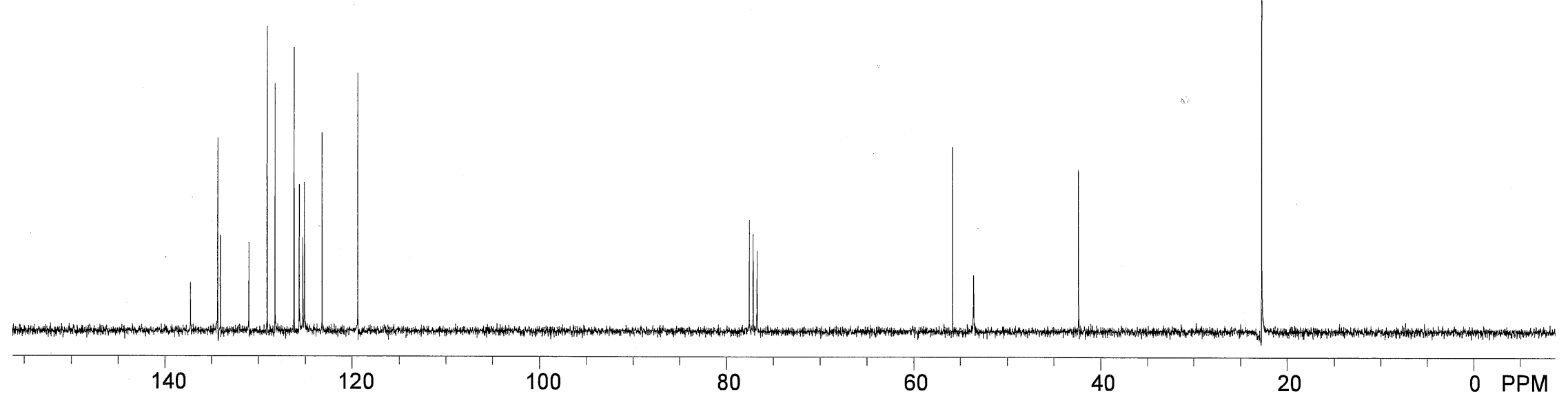




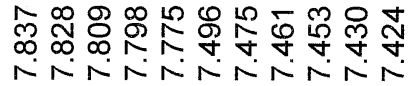

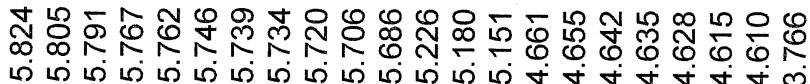

$1+1<1<1)$

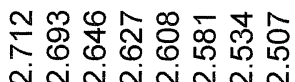

$1+1+1$

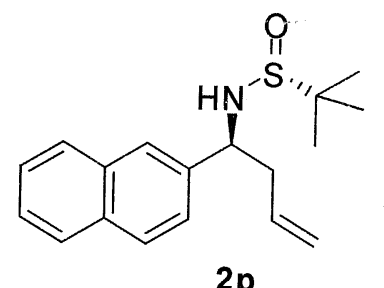

$2 p$

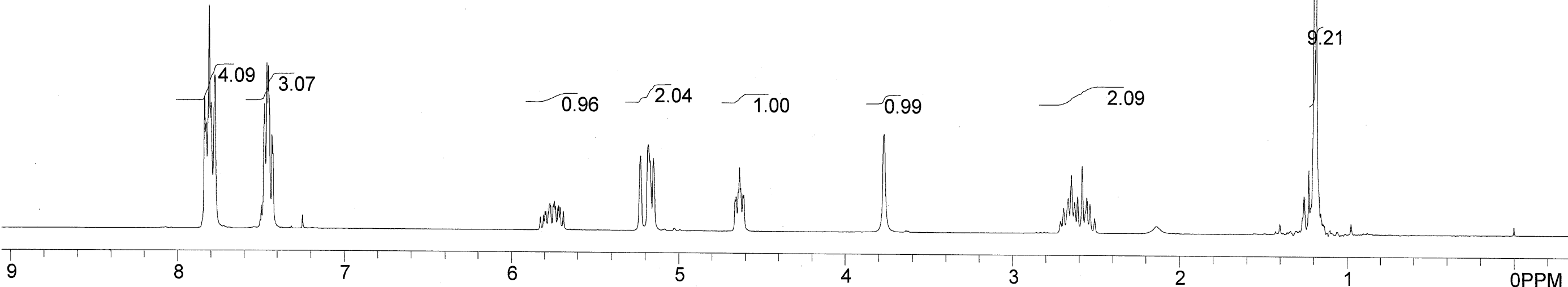




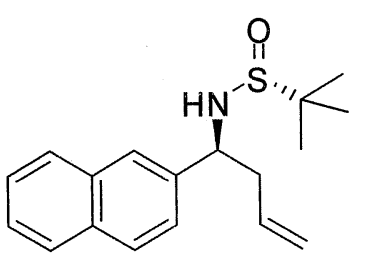

$2 p$

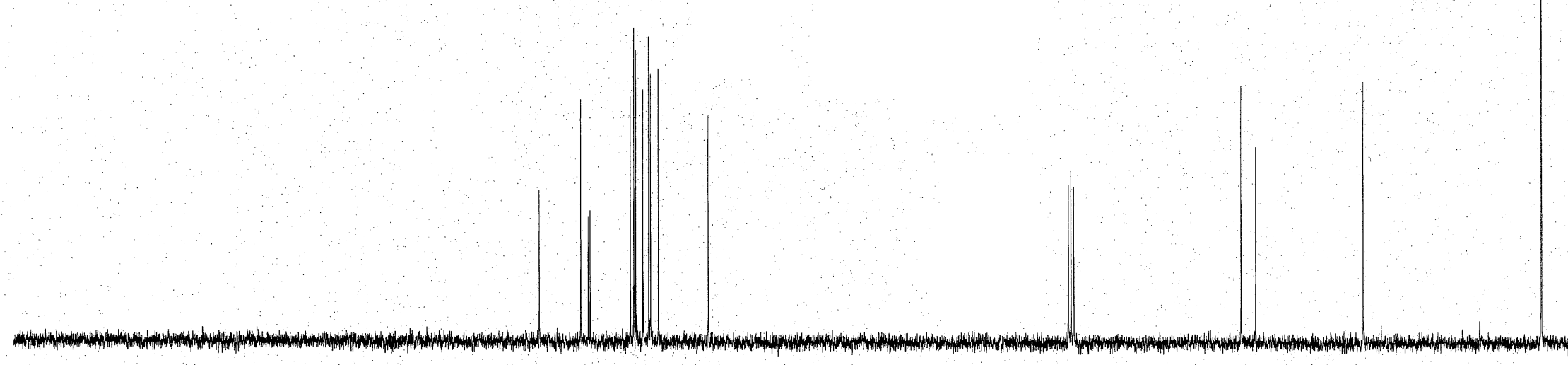




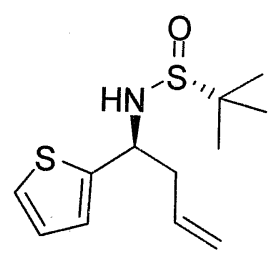

$2 q$
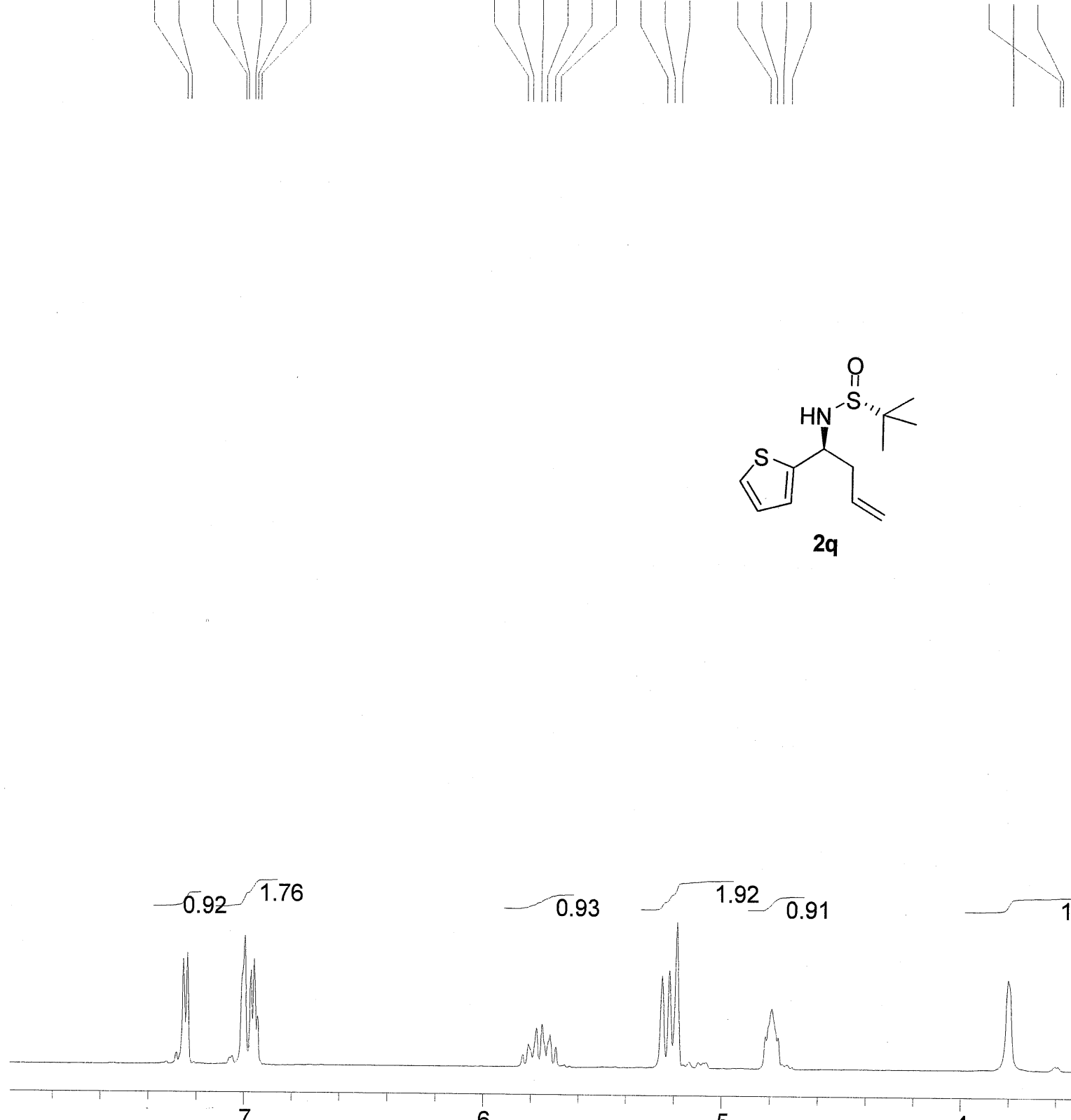


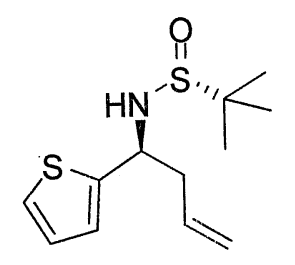

$2 q$

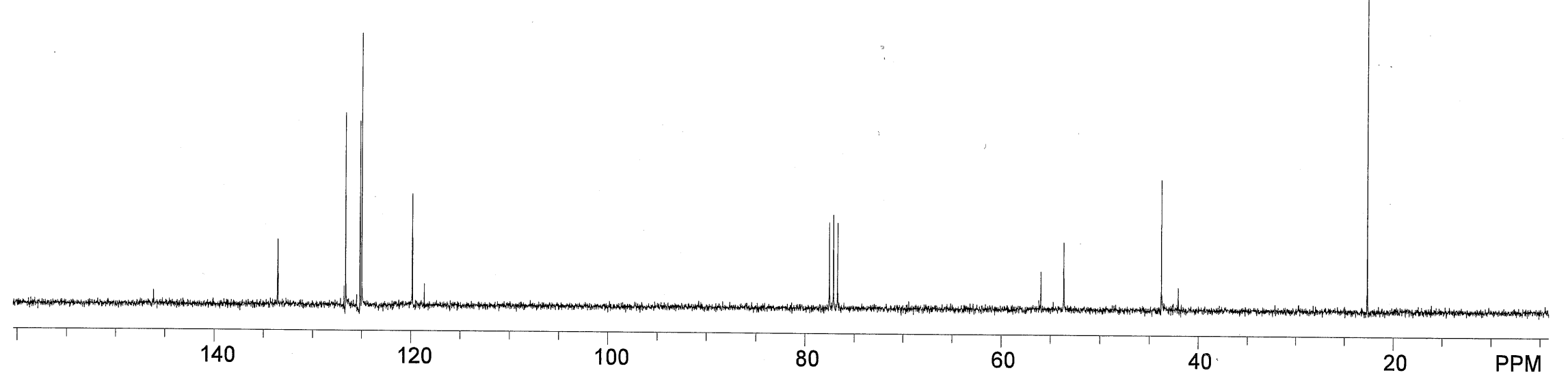



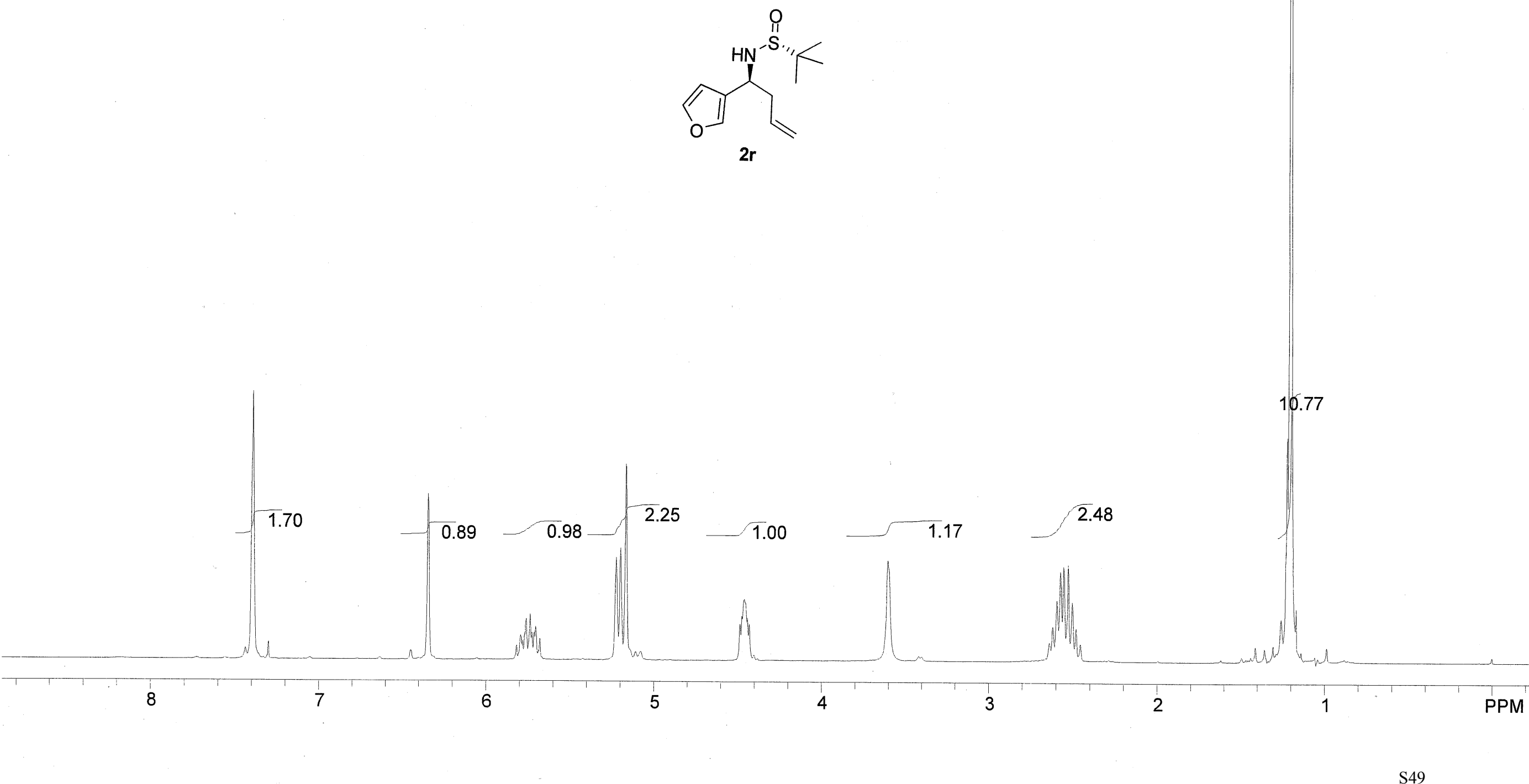

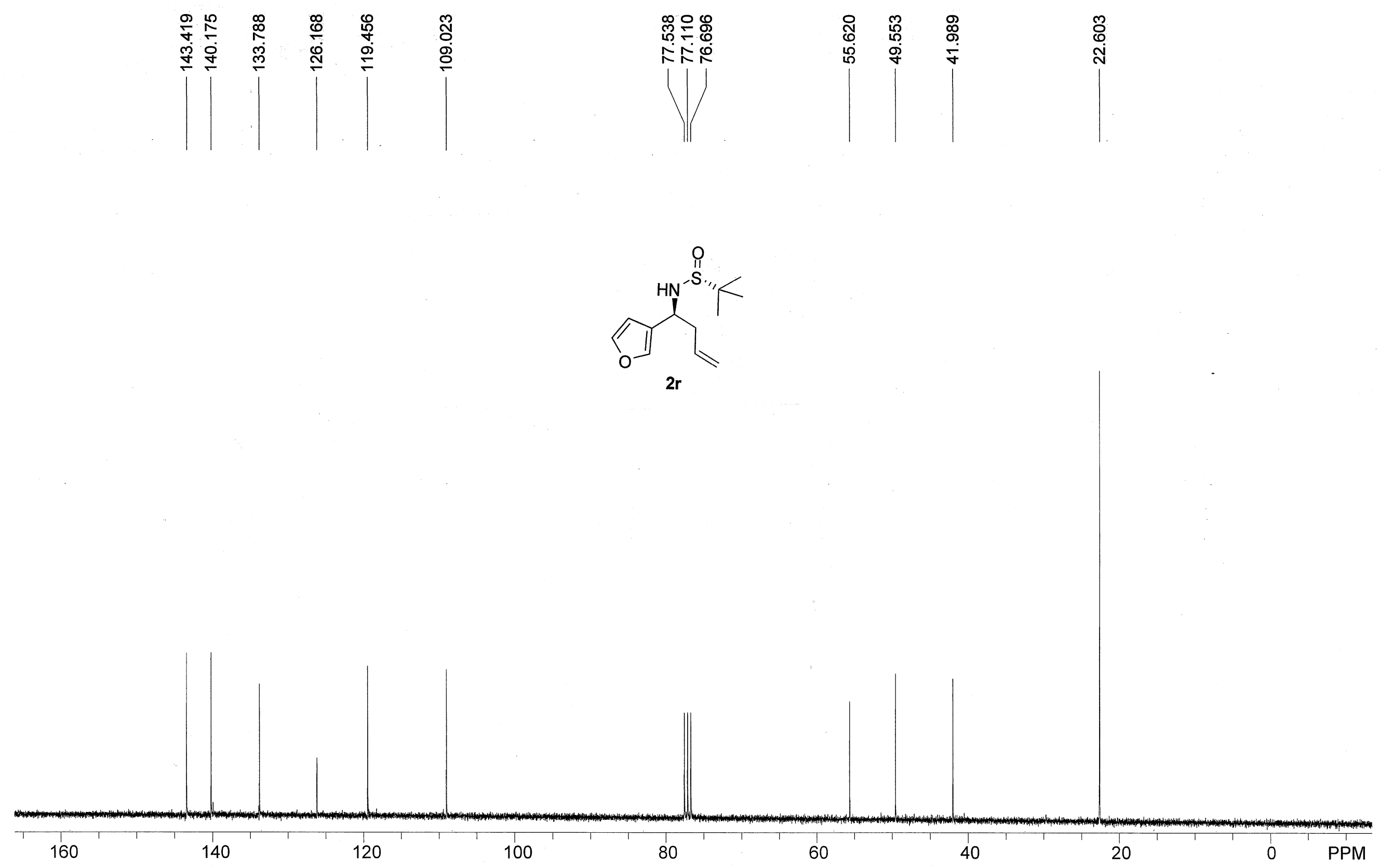


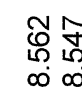

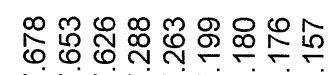

只只卡卡

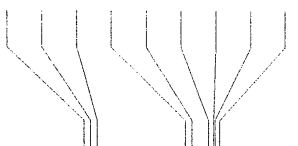

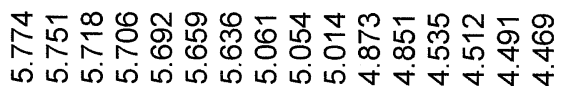

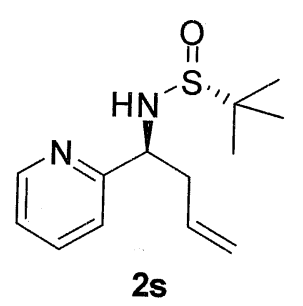

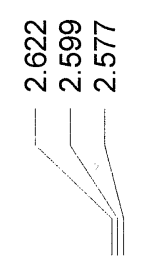

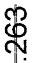

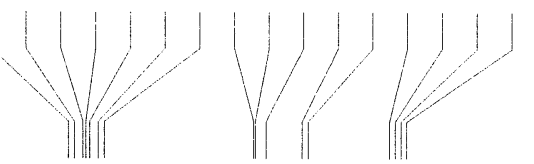

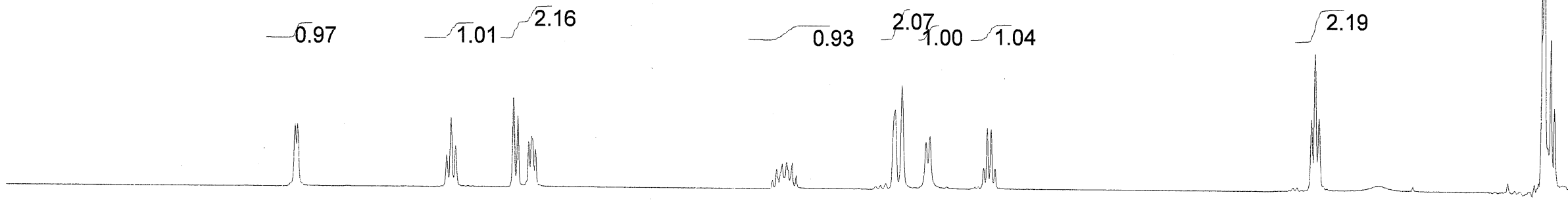




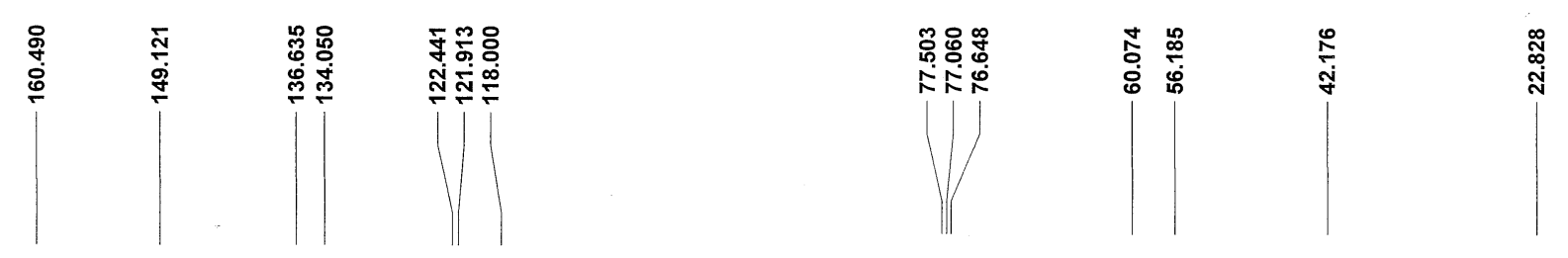

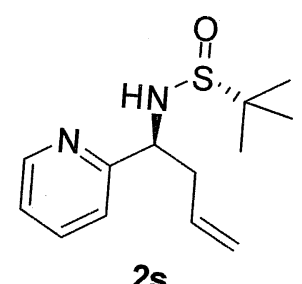

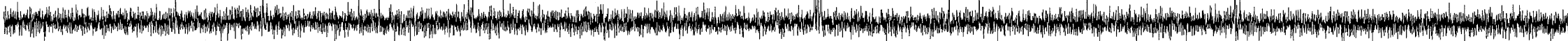




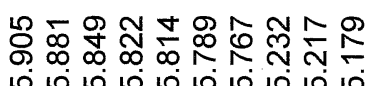

11111

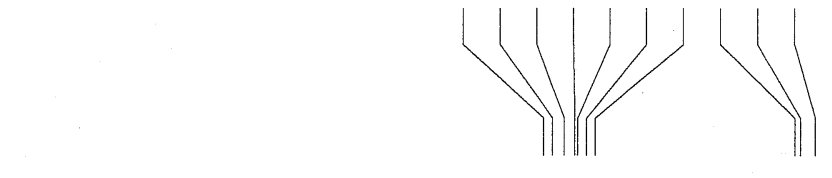

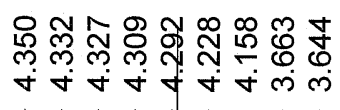

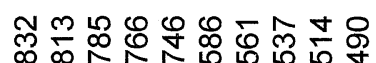

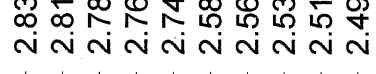

-
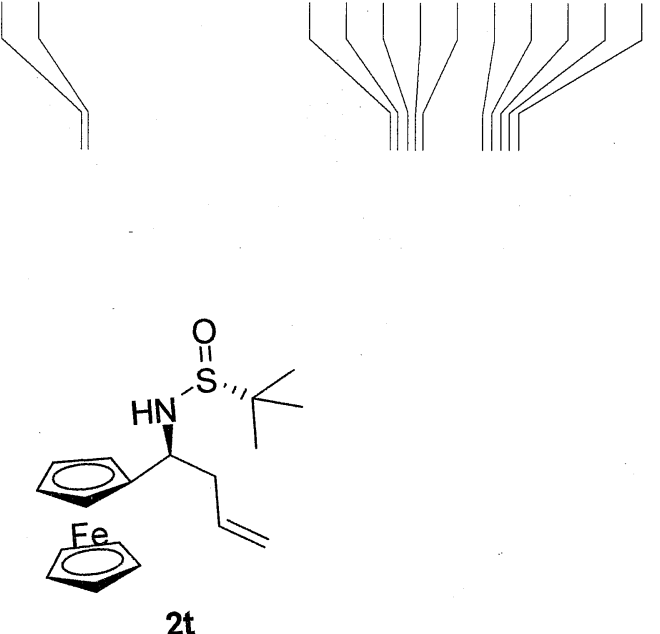

8.03

9103

\lrcorner

6

5

4

$-0.96$

$\sqrt{1.01} 1.01$

me doh

PPM 


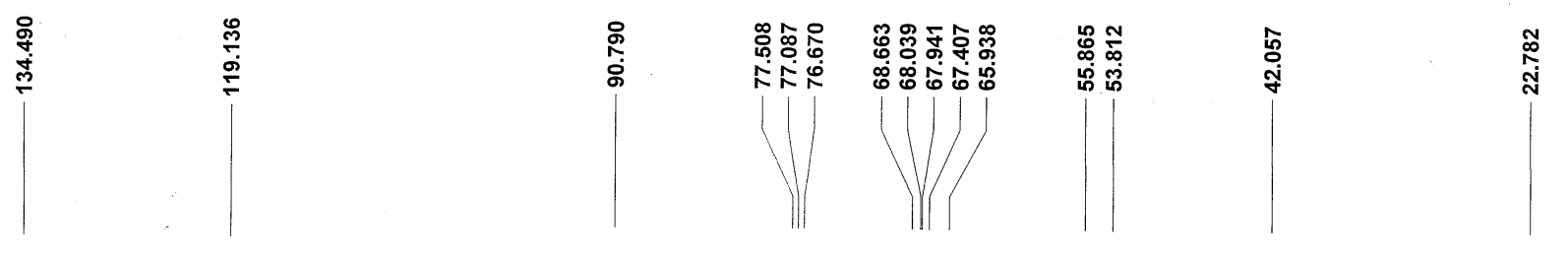

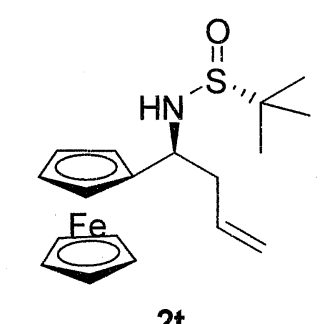

$2 t$

mow 


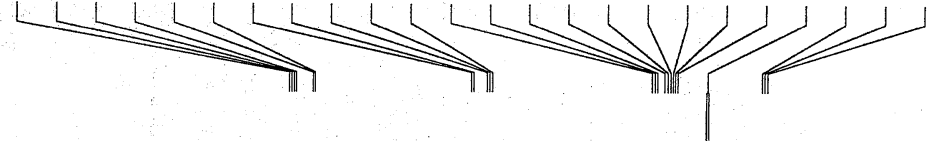

Current Data Parameters Current
NAME
EXNO PROCNO

F2 - Acquisition Parameter

Date_ 20061218

$\begin{array}{lr}\text { Time } & 16.09 \\ \text { INSTRUM } & \text { av500 }\end{array}$

$\begin{array}{lr}\text { INSTRUM } & \text { av500 } \\ \text { PROBHD } 5 \mathrm{~mm} \text { TXO } 19 \mathrm{~F} / 1\end{array}$

\begin{tabular}{rrr} 
PROBHD & $5 \mathrm{~mm}$ TXO \\
PULPROG & 19F/1 \\
\hline
\end{tabular}

TD

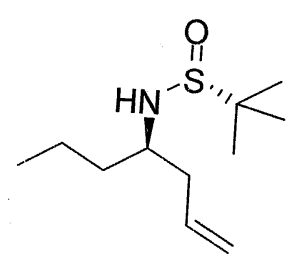

SOLVENT

NS

DS

SWH $\quad 15015.015$

FIDRES $\quad 0.250017 \mathrm{~Hz}$

AQ $\quad 1.9999481 \mathrm{sec}$

DW

$2 \mathrm{u}$

DE $\quad 6.50$ usec

D1 $2.0000000 \mathrm{~K}$

$=== \pm===$ CHANNEL $f 1$ =s==s==

NUC1 $1 \mathrm{H}$

P1
PL1

SF01 $500.1321411 \mathrm{MHz}$

F2 - Processing parameters

$\begin{array}{cc}\text { SI } & 65536 \\ \text { SF } & 500.1299982 \mathrm{MHZ}\end{array}$

WDW

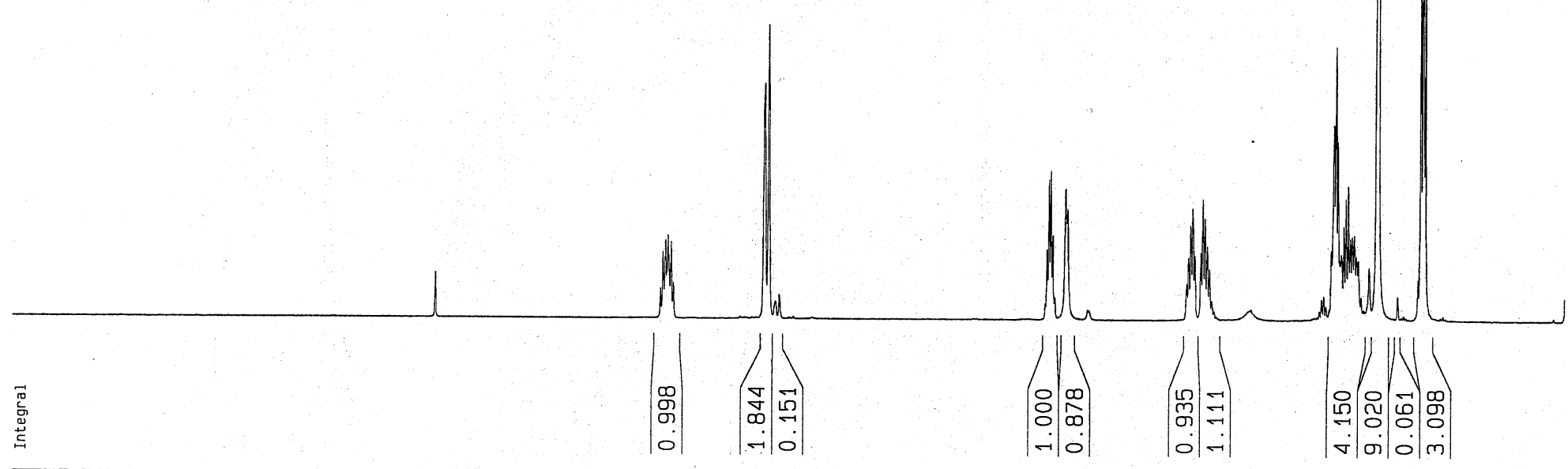

SSB

LB $\quad 0.00 \mathrm{~Hz}$

$\begin{array}{lr}\text { GB } & 0 \\ P C & 1.00\end{array}$

10 NMR plot parameters

CX $\quad 20.00 \mathrm{~cm}$

$\begin{array}{ll}\mathrm{CY} & 0.00 \mathrm{~cm}\end{array}$

F1P $\quad 10.000 \mathrm{ppm}$

F1 $\quad 5001.30 \mathrm{~Hz}$

F2P $\quad 0.000 \mathrm{ppm}$

$\quad 0.00 \mathrm{~Hz}$

$\begin{array}{lr} & 0.50000 \mathrm{ppm} / \mathrm{cm} \\ \mathrm{HZCM} & 250.06500 \mathrm{~Hz} / \mathrm{cm}\end{array}$ 


\begin{tabular}{|c|c|c|c|}
\hline 员 & 쏨 & 怘 종 & 等 号 \\
\hline$\stackrel{\infty}{\exists}$ & $\begin{array}{l}\text { 용ㅇ } \\
U j\end{array}$ & & $\dot{\rho}$ \\
\hline
\end{tabular}

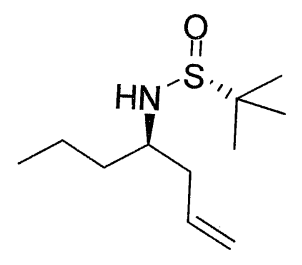

$2 u$

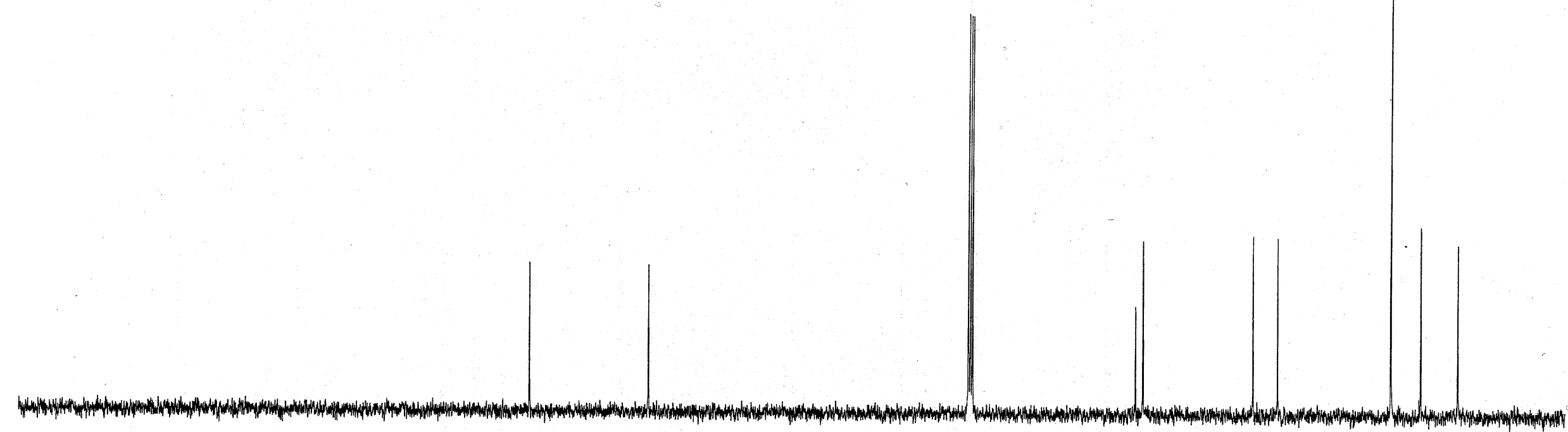




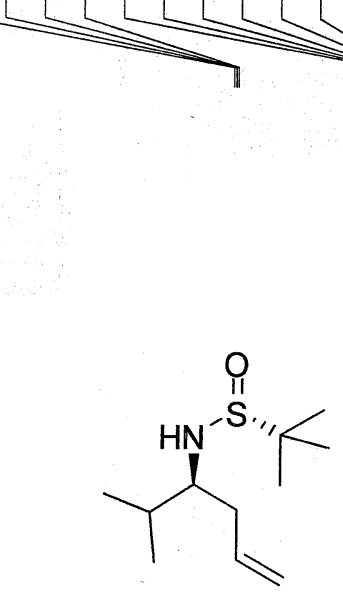

2v

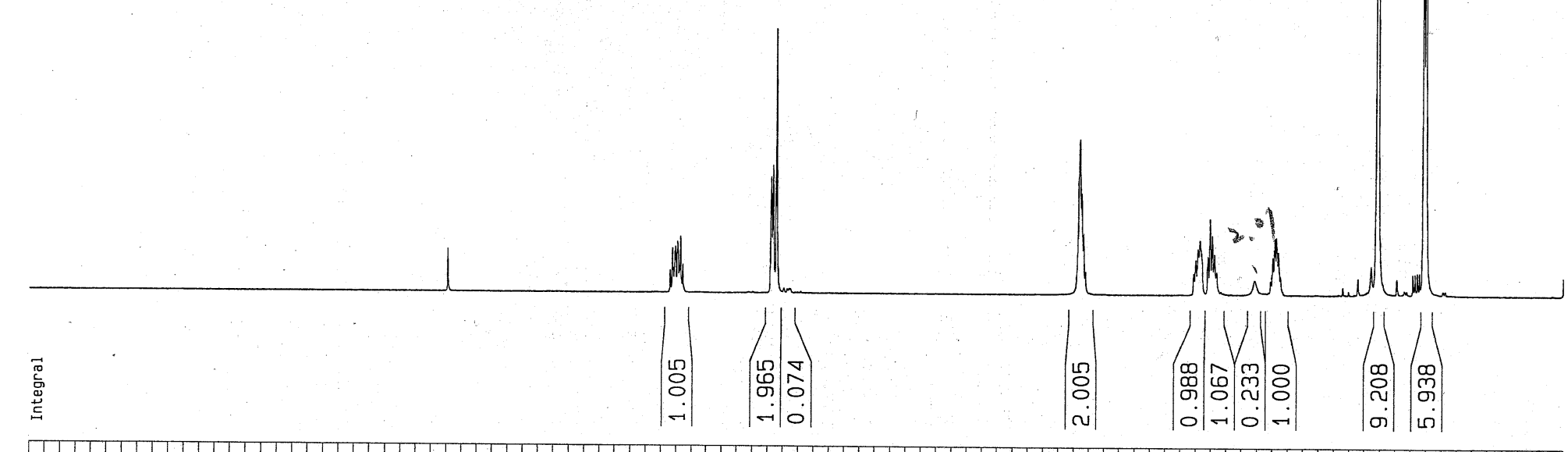

Current Data Parameters NAME

EXPNO

2 - Acquisition Parameter

Date_ $\quad 20061219$

Time

8.26

av500
INSTRUM

PROBHD $5 \mathrm{~mm}$ TXO 19F/

$\begin{array}{lr}\text { PULPROG } & 29 \\ \text { TD } & 60056\end{array}$

$\begin{array}{ll}\text { TD } & 60056 \\ \text { SOLVENT } & \text { CDC13 }\end{array}$

SWH $\quad 15015.015 \mathrm{~Hz}$

FIDRES $\quad 0.250017 \mathrm{~Hz}$

AQ $\quad 1.9999481 \mathrm{sec}$

DW

DE

33.300 usec

6.50 us

$200000 \mathrm{~K}$

$=======$ CHANNEL $f 1=======$
NUC1
$1 \mathrm{H}$

$\begin{array}{ll}\text { NUC1 } & 1 \mathrm{H} \\ \text { P1 } & 4.00 \text { usec }\end{array}$

$\begin{array}{ll}\text { P1 } & 4.00 \text { usec } \\ \text { PL1 } & 0.00 \mathrm{~dB}\end{array}$

SF01 $\quad 500.1321411 \mathrm{MHz}$

F2 - Processing parameters

SI Process jng Dara

$\begin{array}{ll}\text { SF } & 500.1299989 \mathrm{MHz}\end{array}$

WDW no

$\begin{array}{ll}\text { LB } & 0 \\ & 0.00 \mathrm{~Hz}\end{array}$

$\begin{array}{ll}\text { GB } & 0.00 \\ P C & 1.00\end{array}$

10 NMR plot parameters

CX $\quad 0.00 \mathrm{~cm}$

$\begin{array}{ll}\mathrm{CY} & 0.00 \mathrm{~cm}\end{array}$

F1P $\quad 10.000 \mathrm{ppm}$

F1 $\quad 5001.30 \mathrm{~Hz}$

F2P $\quad 0.000 \mathrm{ppm}$

$\quad 0.00 \mathrm{~Hz}$

PPMCM

$0.50000 \mathrm{ppm} / \mathrm{c}$

$250.06500 \mathrm{~Hz} / \mathrm{cm}$ 


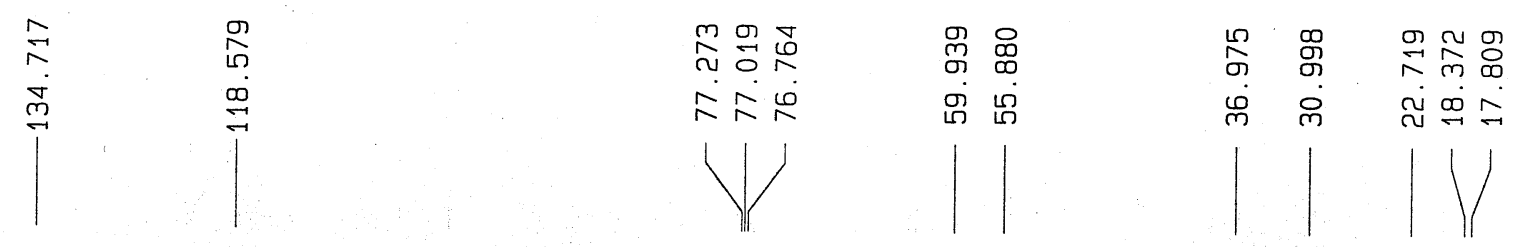

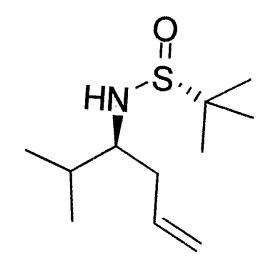

$2 v$

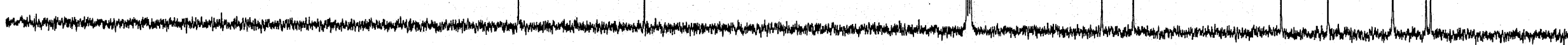




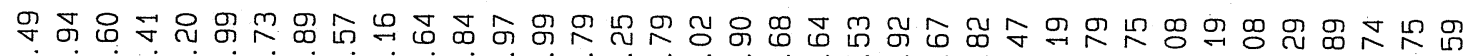

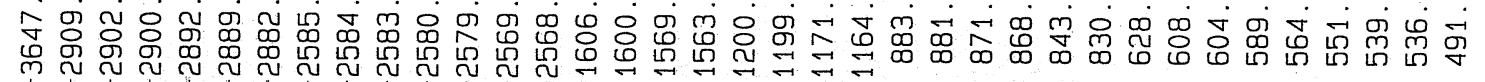
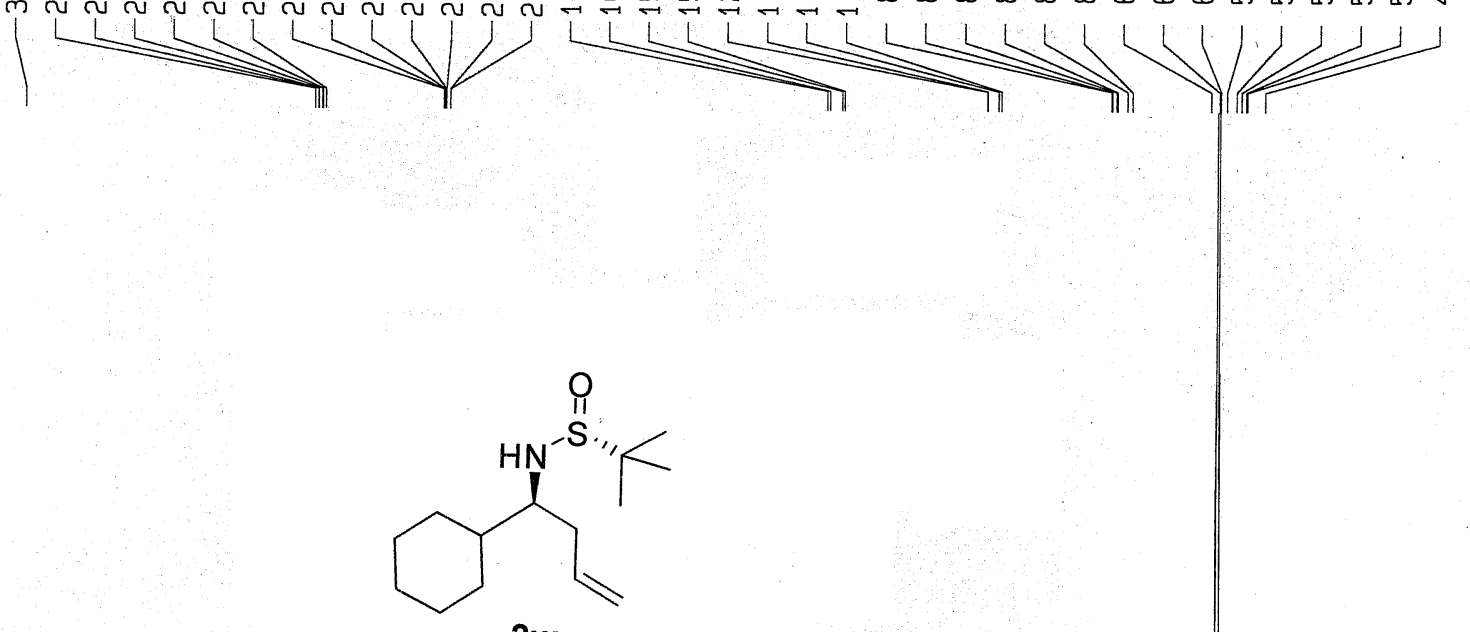

$2 w$

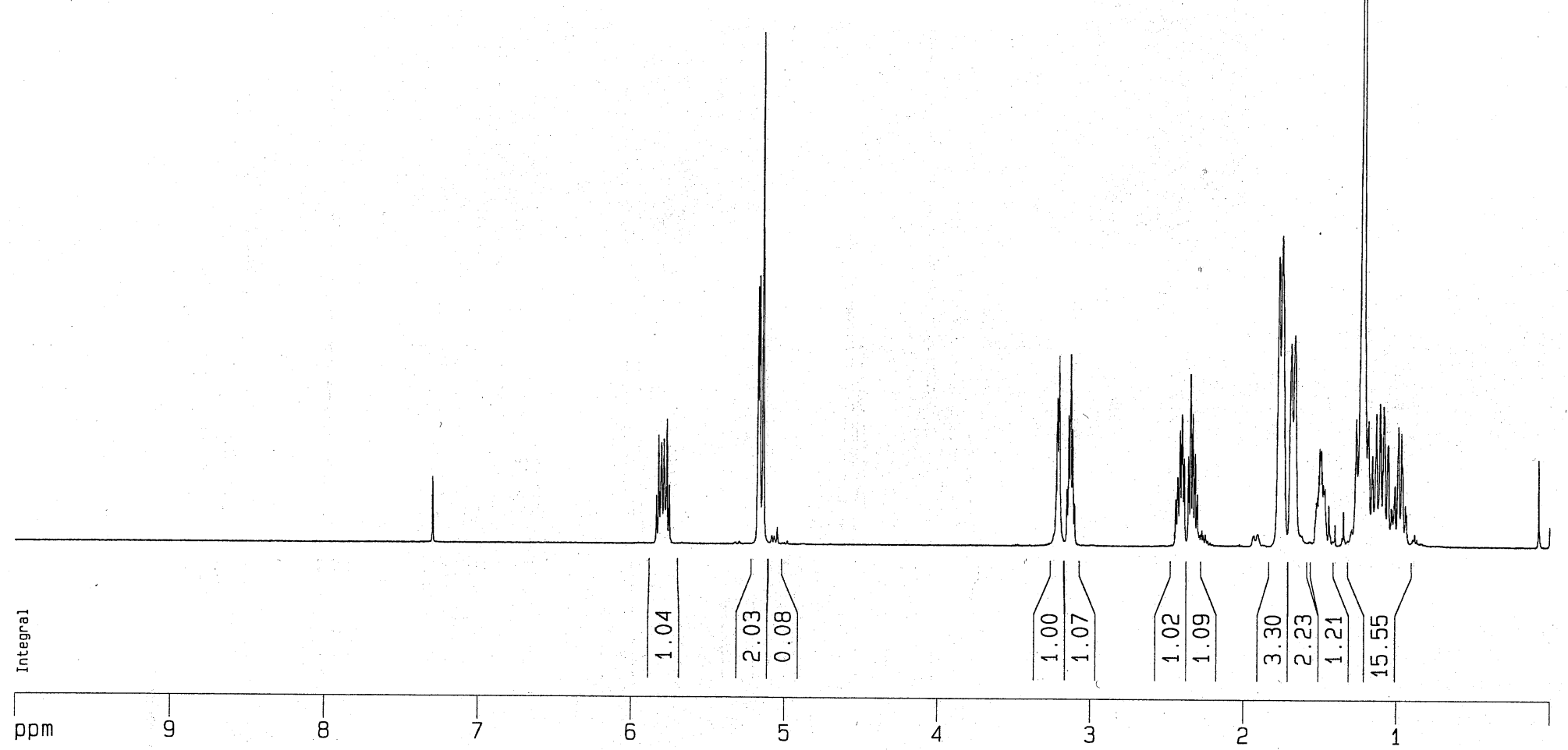

Current Data Parameters

NAME

PROCNO

$\mathrm{txo}$
1

F2 - Acquisition Parameters

Date $\quad 20061218$

$\begin{array}{ll}\text { Time } & 16.21 \\ \text { INSTRUM } & \text { av500 }\end{array}$

$\begin{array}{lr}\text { INSTRUM } & \text { av500 } \\ \text { PROBHD } 5 \mathrm{~mm} \text { TXO } 19 \mathrm{~F} / 1\end{array}$

$\begin{array}{lr}\text { PULPROG } & 29 \\ \text { TD } & 60056\end{array}$

SOLVENT

NS

FIDRES $\quad 15015.015 \mathrm{~Hz}$

AQ $\quad 1.9999481 \mathrm{sec}$

$D W$

DE

64
33.300 usec

6.50 use

$300.0 \mathrm{~K}$

$=======$. CHANNEL $f 1$ ========

$\begin{array}{ll}\text { NUC1 } & 1 \mathrm{H} \\ \mathrm{P} 1 & 4.00 \mathrm{usec}\end{array}$

$\begin{array}{lr}\text { PL1 } & 0.00 \mathrm{~dB} \\ & 500.1321411 \mathrm{dHz}\end{array}$

F2 - Processing parameters

SI Processing parameters

SF $\quad 500.1259964 \mathrm{MHz}$

WDW
SSB

LB $\quad 0.00 \mathrm{~Hz}$

$\begin{array}{lr}\text { GB } & 0 \\ P C & 1.00\end{array}$

10 NMP plot parameters

CX $\quad 20.00 \mathrm{~cm}$

CY $\quad 0.00 \mathrm{~cm}$

F1P $\quad 10.000 \mathrm{ppm}$

$1 \quad 5001.30 \mathrm{~Hz}$

F2P $\quad 0.000 \mathrm{ppm}$

$\quad 0.00 \mathrm{~Hz}$

$\begin{array}{lr} & 0.50000 \mathrm{ppm} / \mathrm{cm} \\ \mathrm{HZCM} & 250.06500 \mathrm{~Hz} / \mathrm{cm}\end{array}$ 


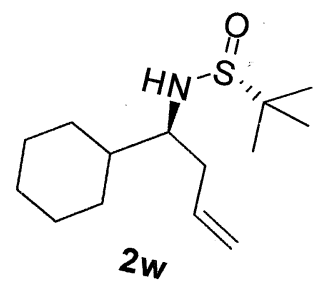

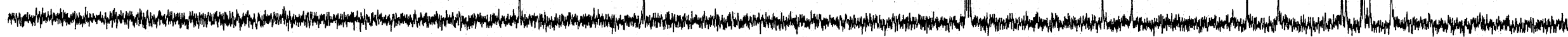



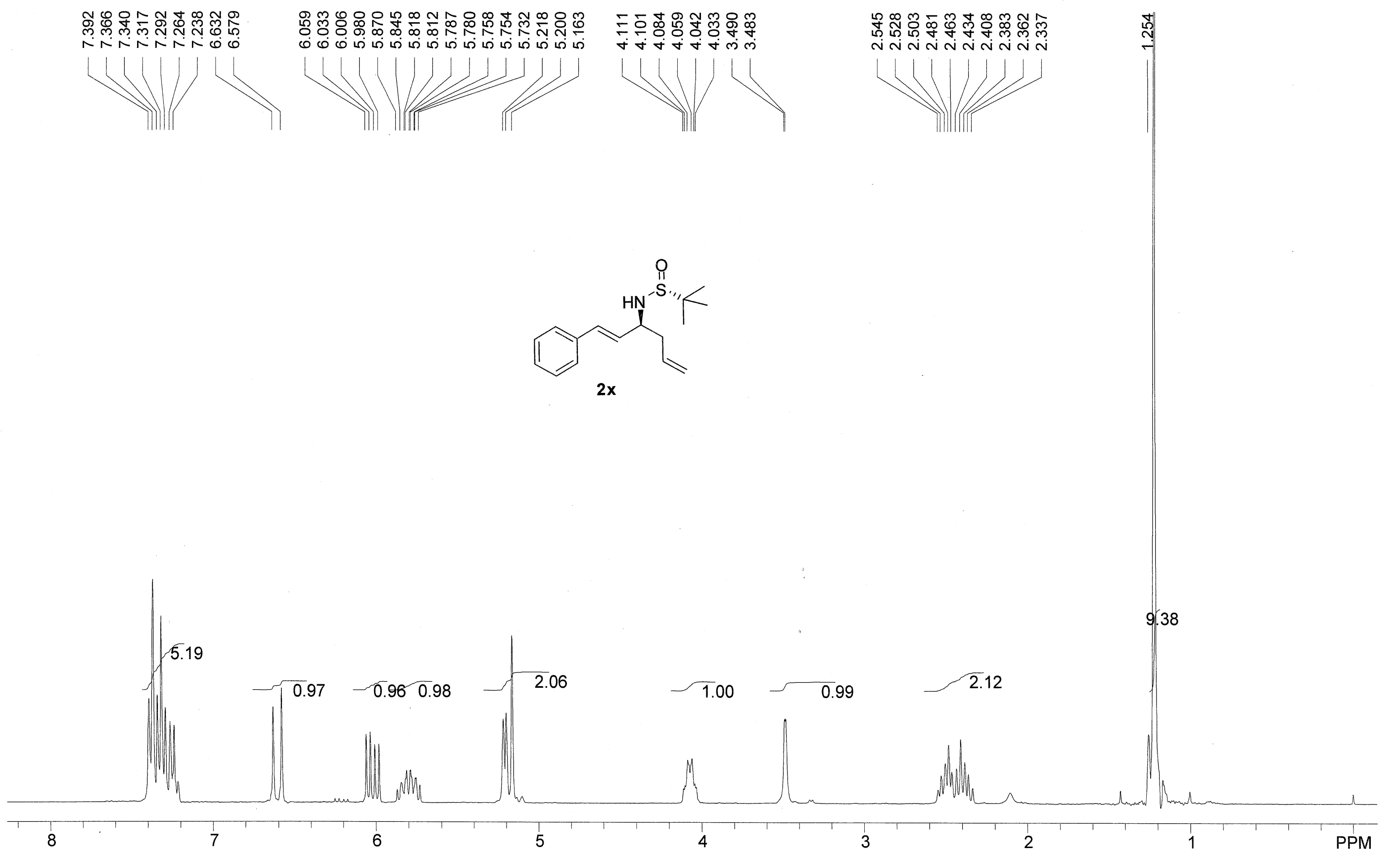


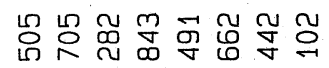

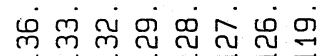

$\vec{\jmath} \vec{i} \vec{j} \vec{j}$

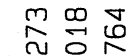

송

VI

$\mid$

品

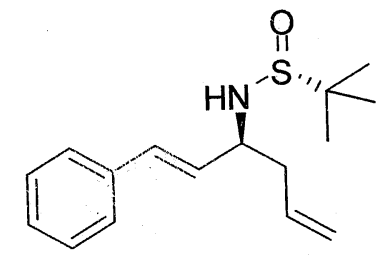

$2 x$

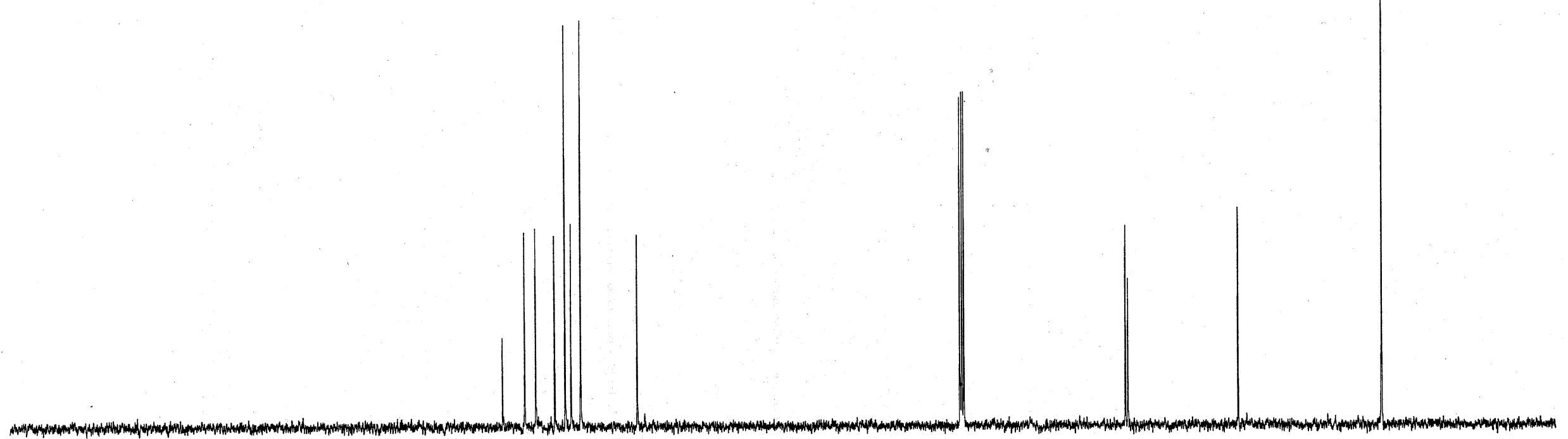




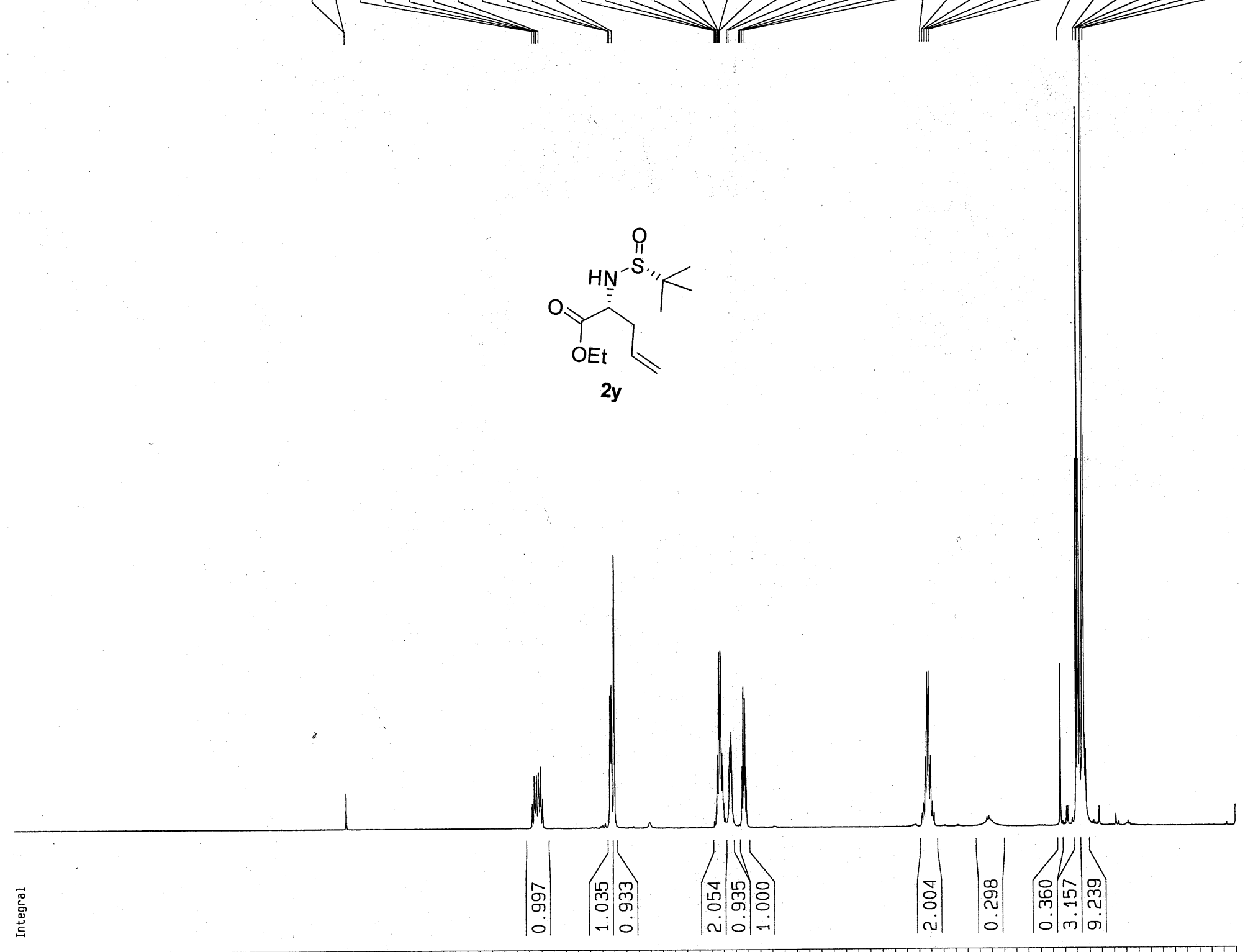

Current Data Parameters

F2 - Acquisition Parameters

Date_ 20061221

Time

B. 29

$\begin{array}{lr}\text { INSTRUM } & \text { av500 } \\ \text { PROBHD } 5 \mathrm{~mm} \text { TXO } & 19 F / 1\end{array}$

PULPROG 29

$\begin{array}{ll}\text { TD } & 60056 \\ \text { SOLVENT } & \text { CDC13 }\end{array}$

SWH $\quad 15015.015 \mathrm{~Hz}$

FIDRES $\quad 0.250017 \mathrm{~Hz}$

AQ $\quad 1.9999481 \mathrm{sec}$

$\begin{array}{lr}R G & 64 \\ D W & 33.300 \\ \text { usec }\end{array}$

DE $\quad 6.50$ use

TE
D1 $2.00000000 \mathrm{sec}$

$=======$ CHANNEL $f 1 \quad=======$

NUC1 $1 \mathrm{H}$

P1 4.00 us

SF01 500.1331411 MHZ

F2 - Processing parameters

SI $\quad 65536$

WDW 500.1

SSB

LB $\quad 0.00 \mathrm{~Hz}$

PC 1.00

10 NMR plot parameters

CX $\quad 20.00 \mathrm{~cm}$

CY $\quad 0.00 \mathrm{~cm}$

$\begin{array}{lr}\text { F1P } & 10.000 \mathrm{ppm} \\ \text { F1 } & 5001.30 \mathrm{~Hz}\end{array}$

FiP $\quad 0.000 \mathrm{ppm}$

F2 $0.00 \mathrm{~Hz}$

PPMCM $\quad 0.50000 \mathrm{ppm} / \mathrm{cm}$

$\mathrm{HZCM} \quad 250.06500 \mathrm{~Hz} / \mathrm{cm}$ 
종웡

송

$\forall$

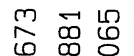

它它它

11
品

m

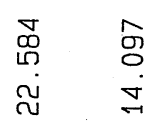

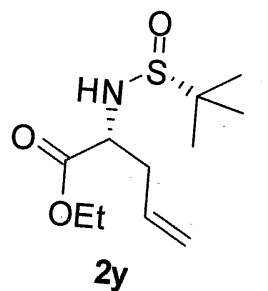

$2 y$

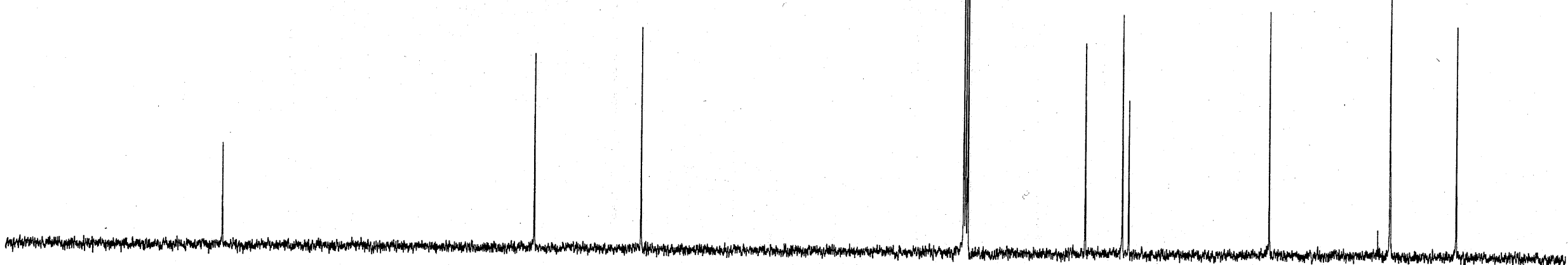




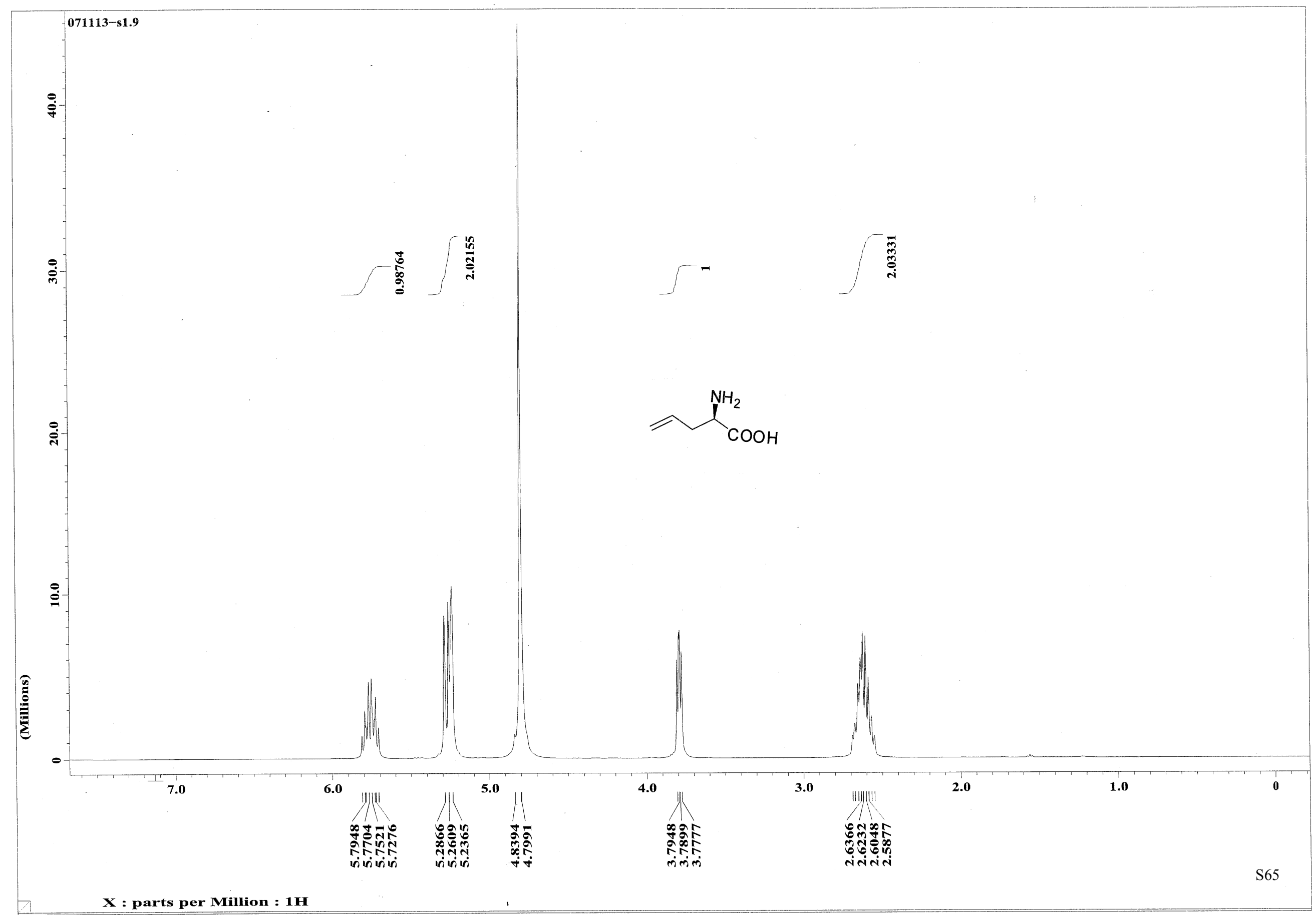




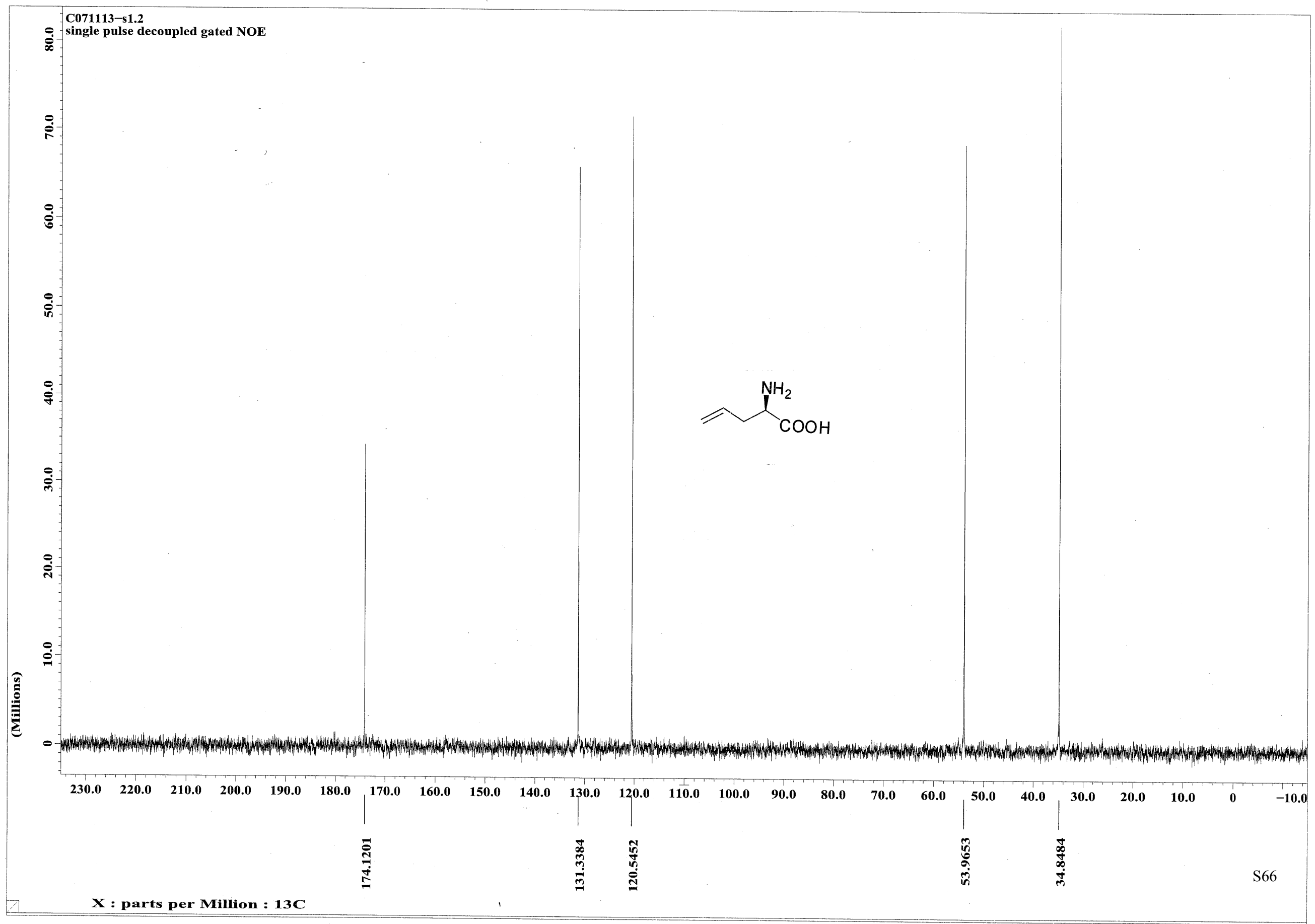




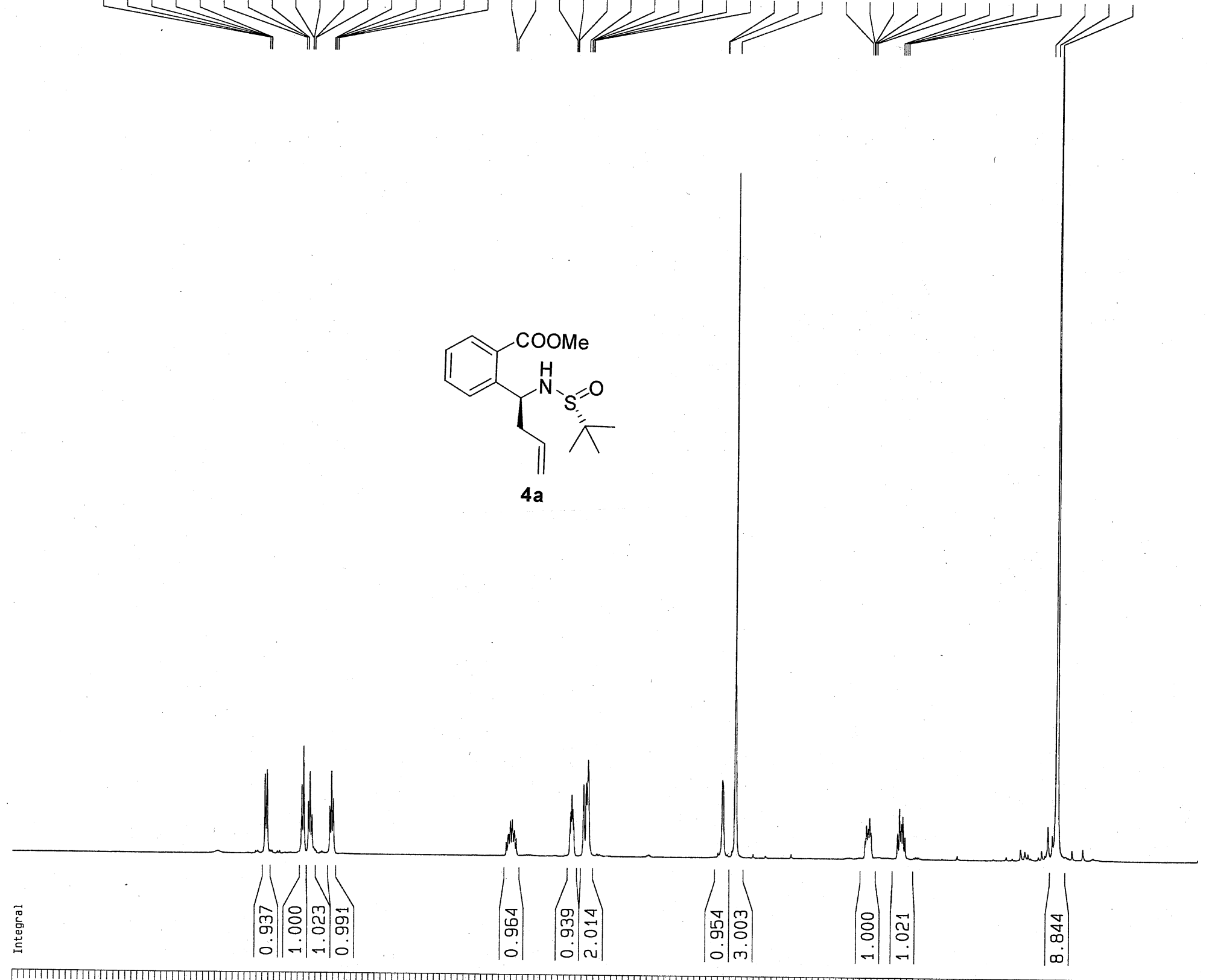

Current Data Parameters NAME EXPNO $\mathrm{t} \times 0$
8
1

F2 - Acquisition Parameters

Date 20061207

Time

INSTRUM 16.20 PROBHD $5 \mathrm{~mm}$ TXO $195 / 1$ PULPROG 29
29 To

SOLVENT

NS

$\begin{array}{lr}\text { DS } & 0 \\ \text { SWH } & 15015.015 \mathrm{~Hz} \\ \text { FIDRES } & 0.229111 \mathrm{~Hz}\end{array}$

AQ $0.229111 \mathrm{~Hz}$

RG $2.1824322 \mathrm{sec}$

DW $\quad 33.300$ usec

DE $\quad 6.50$ usec

$\begin{array}{rr} & 300.0 \mathrm{~K} \\ & 2.00000000\end{array}$

$=======$ CHANNEL $f 1$ = $======$

$\begin{array}{ll}\text { NUC1 } & 1 \mathrm{H} \\ \mathrm{P} 1 & 4.00 \mathrm{usec}\end{array}$

$\begin{array}{ll}\text { P1 } & 4.00 \text { usec } \\ \text { PL1 } & 0.00 \mathrm{~dB}\end{array}$

$\begin{array}{lr}\text { PL1 } & 0.00 \mathrm{~dB} \\ \text { SF01 } & 500.1320806 \mathrm{MHz}\end{array}$

F2 - Processing parameters

F2 - Processing parameters
SI

$\begin{array}{lc}\text { SI } & 65536 \\ \text { SF } & 500.1299925 \\ \text { MHz }\end{array}$

$\begin{array}{lr}\text { SF } & 500.1299925 \\ \text { WDW } & \text { no }\end{array}$

$\begin{array}{lc}\text { SSB } & 0 \\ \text { LB } & 0.00 \mathrm{~Hz}\end{array}$

$\begin{array}{rr}\text { GB } & 0.00 \\ \text { PB } & 0\end{array}$

10 NMR plot parameters

cX NMR plot parameters

CX $\quad 20.00 \mathrm{~cm}$

$\begin{array}{lr}\text { CY } & 0.00 \mathrm{~cm} \\ \text { FIP } & 10.000 \mathrm{ppm}\end{array}$

F1 $\quad \begin{aligned} & 10.000 \mathrm{ppm} \\ & \text { F }\end{aligned}$

$\begin{array}{ll}\text { F2P } & \quad 0.000 \mathrm{ppm}\end{array}$

F2 $\quad 0.00 \mathrm{~Hz}$

HZCM $\quad 0.50000 \mathrm{ppm} / \mathrm{cm}$ 


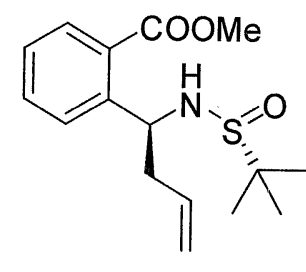

$4 a$

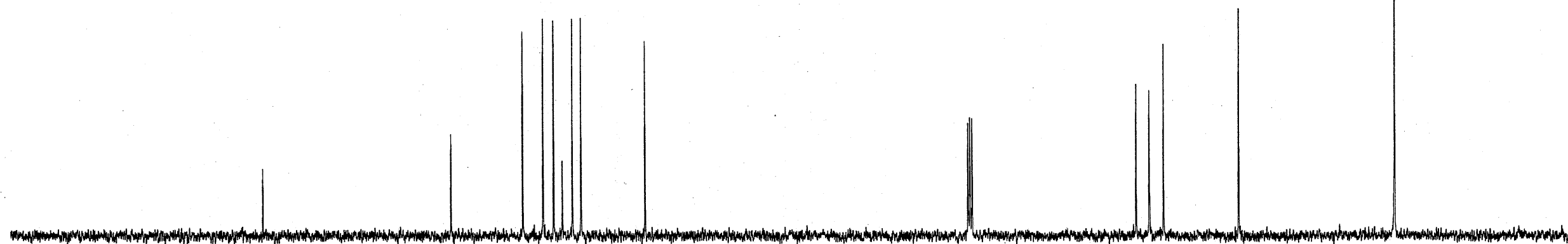



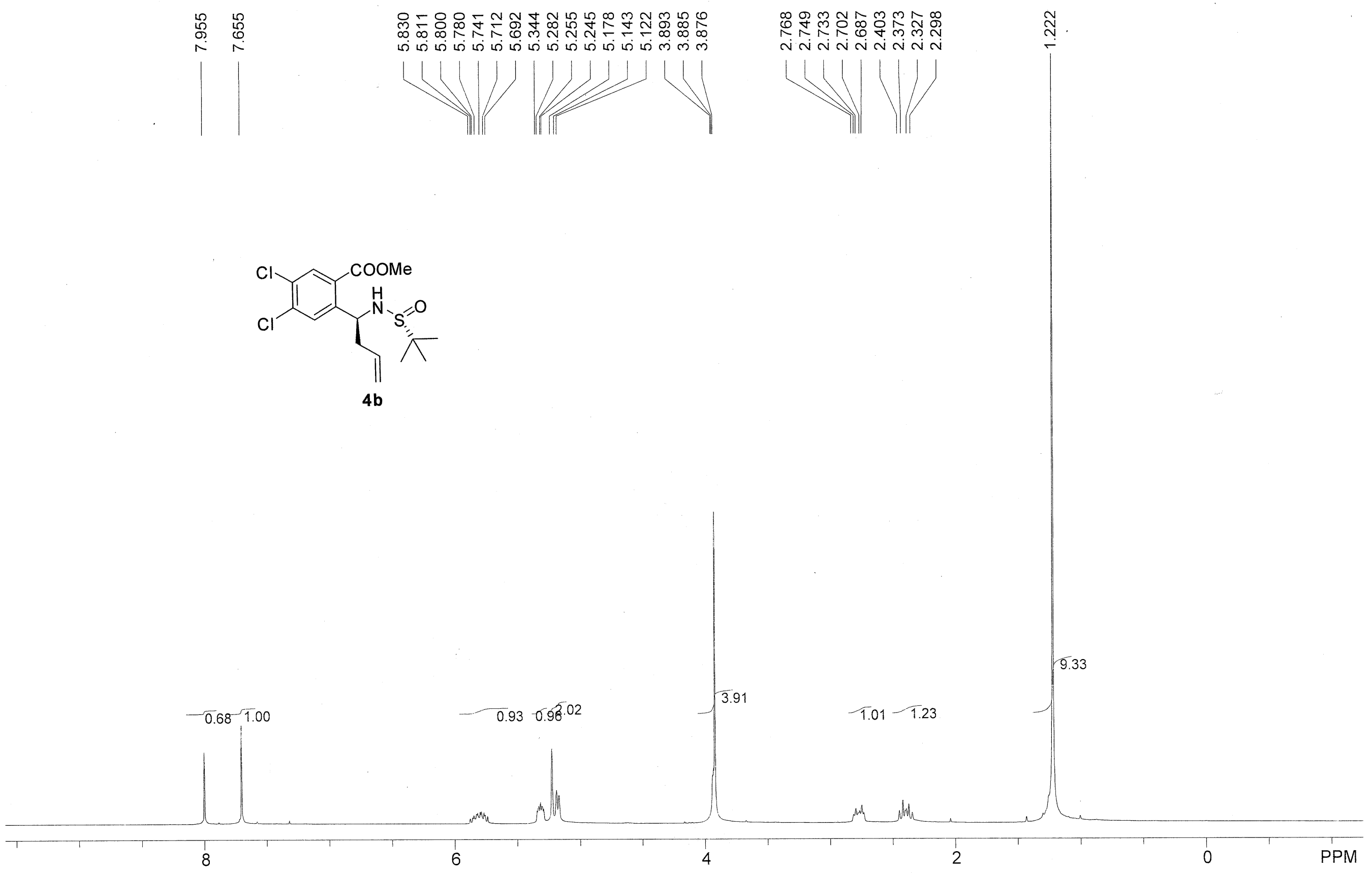


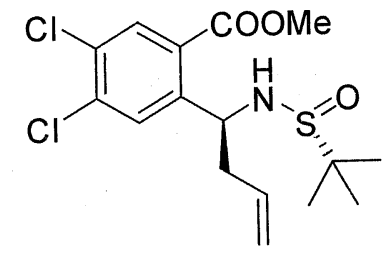

4b

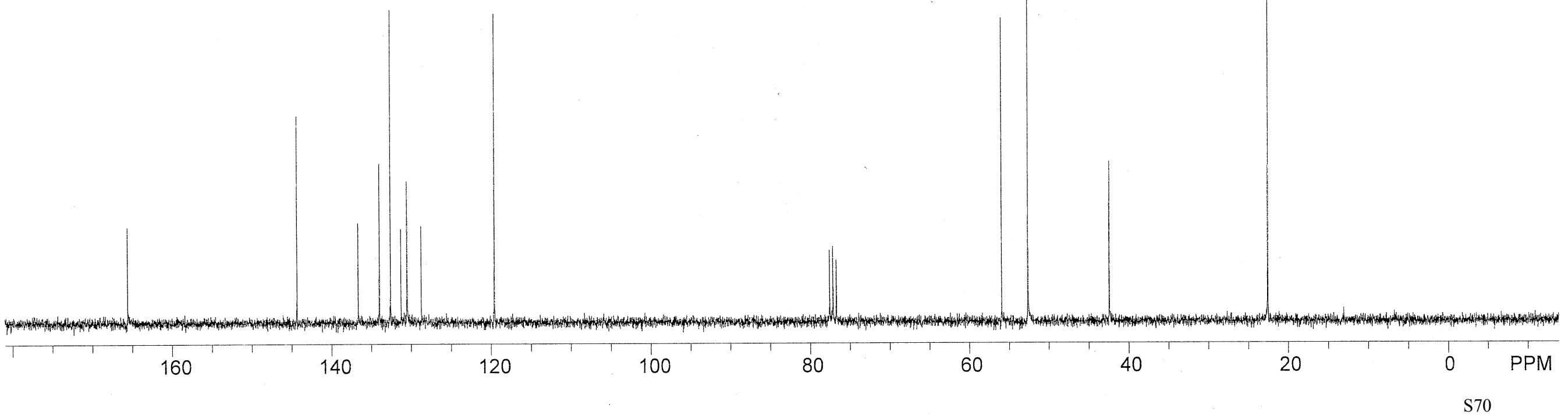




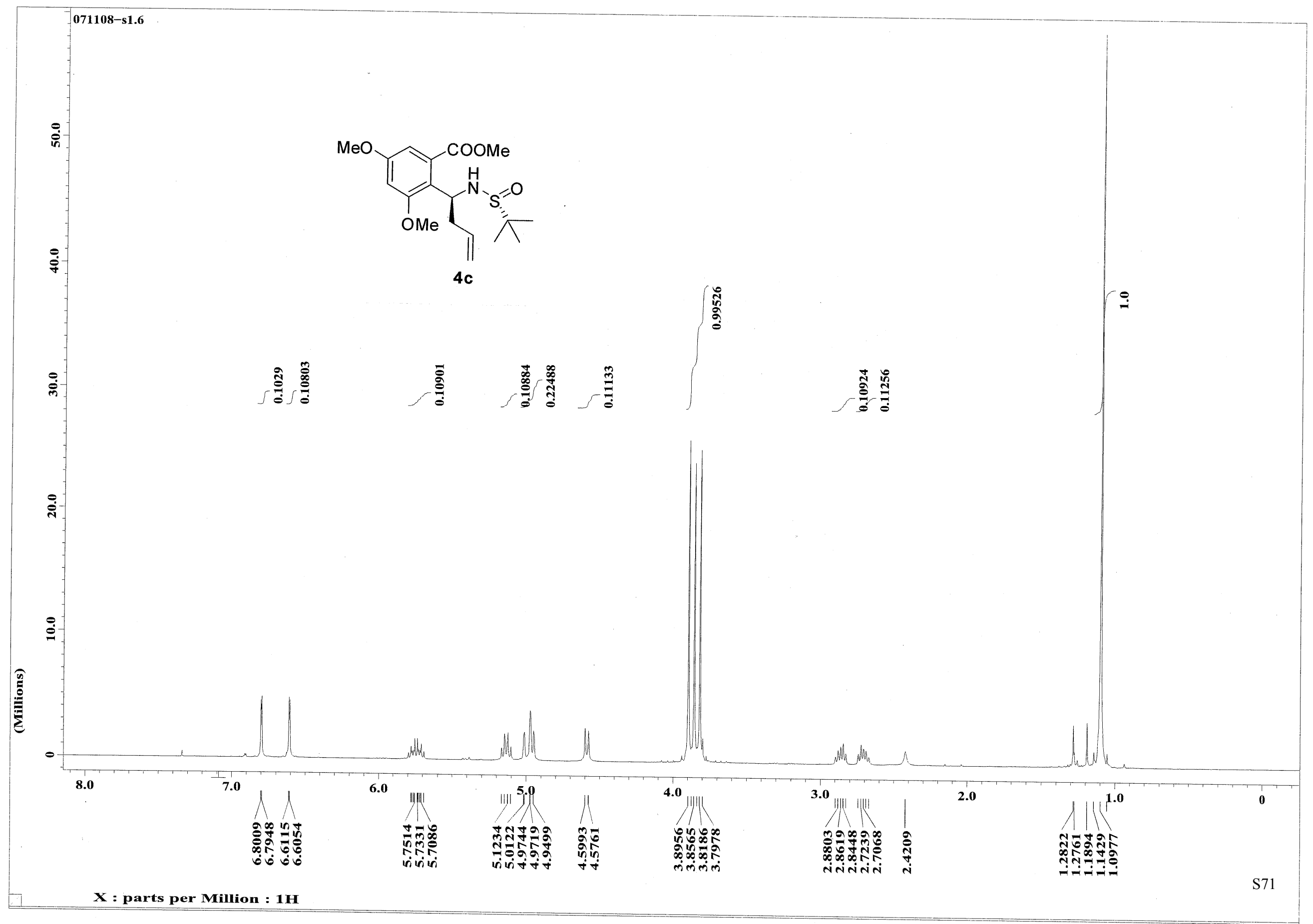




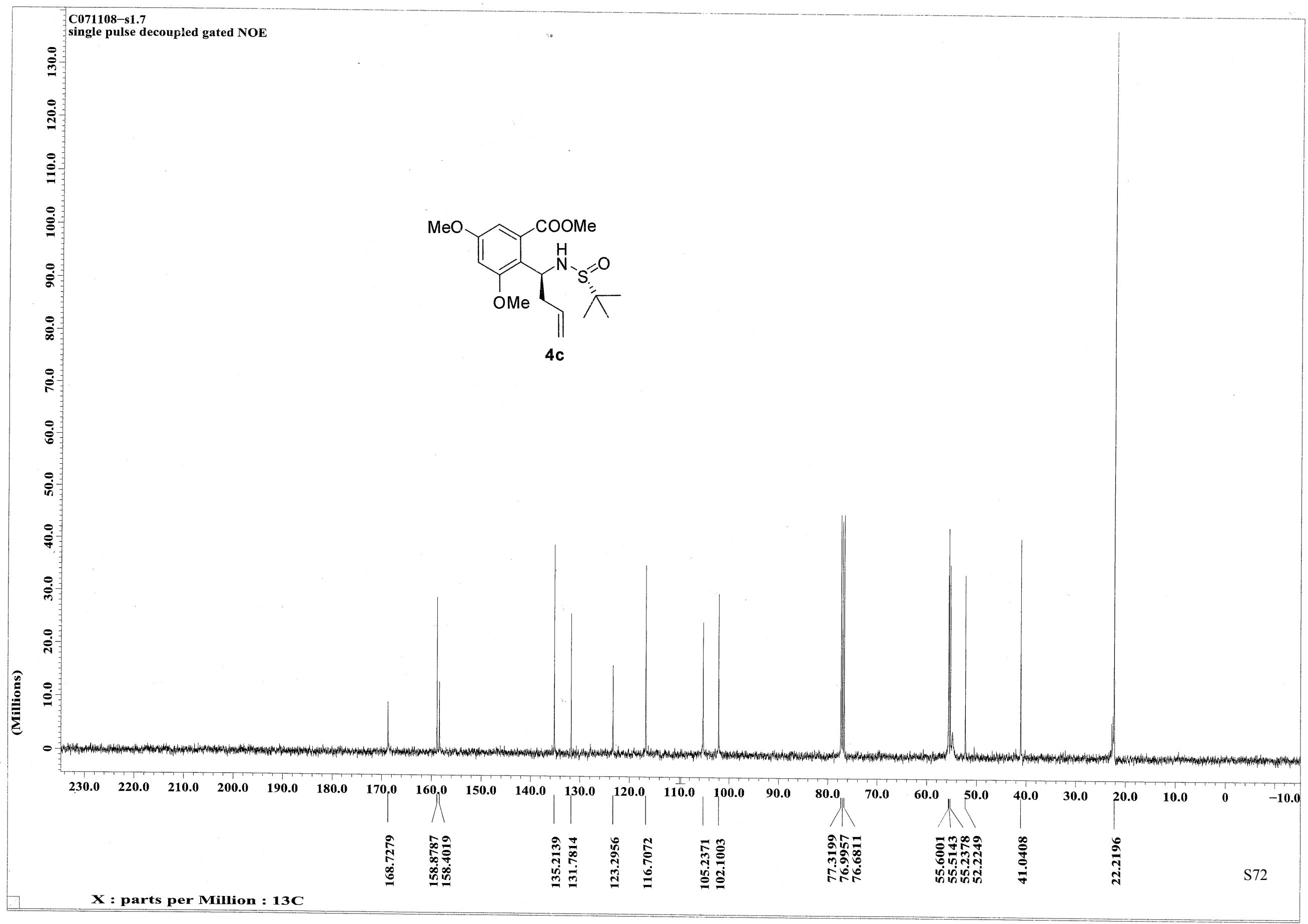




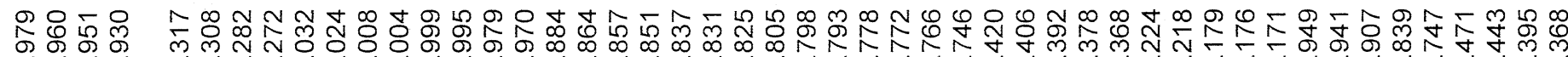

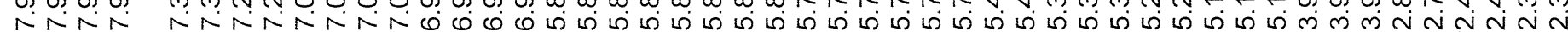
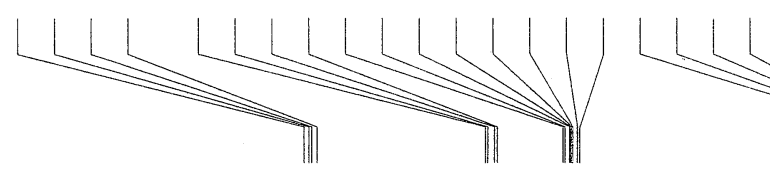

11I
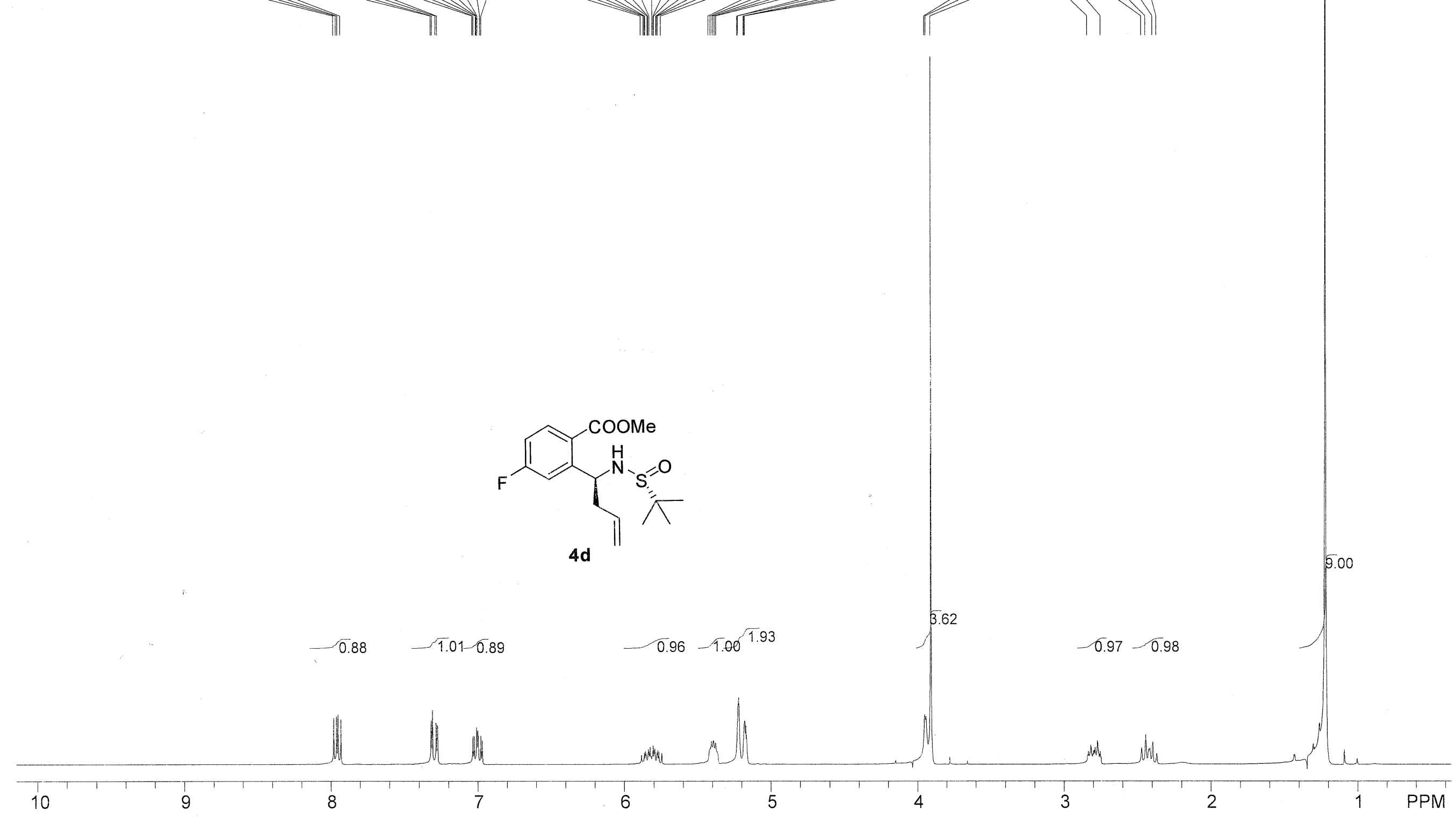


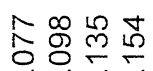

웅

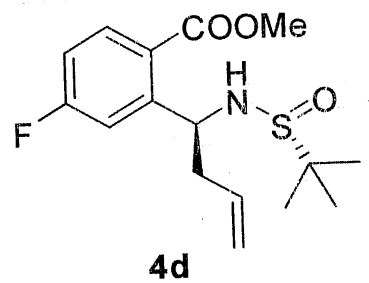



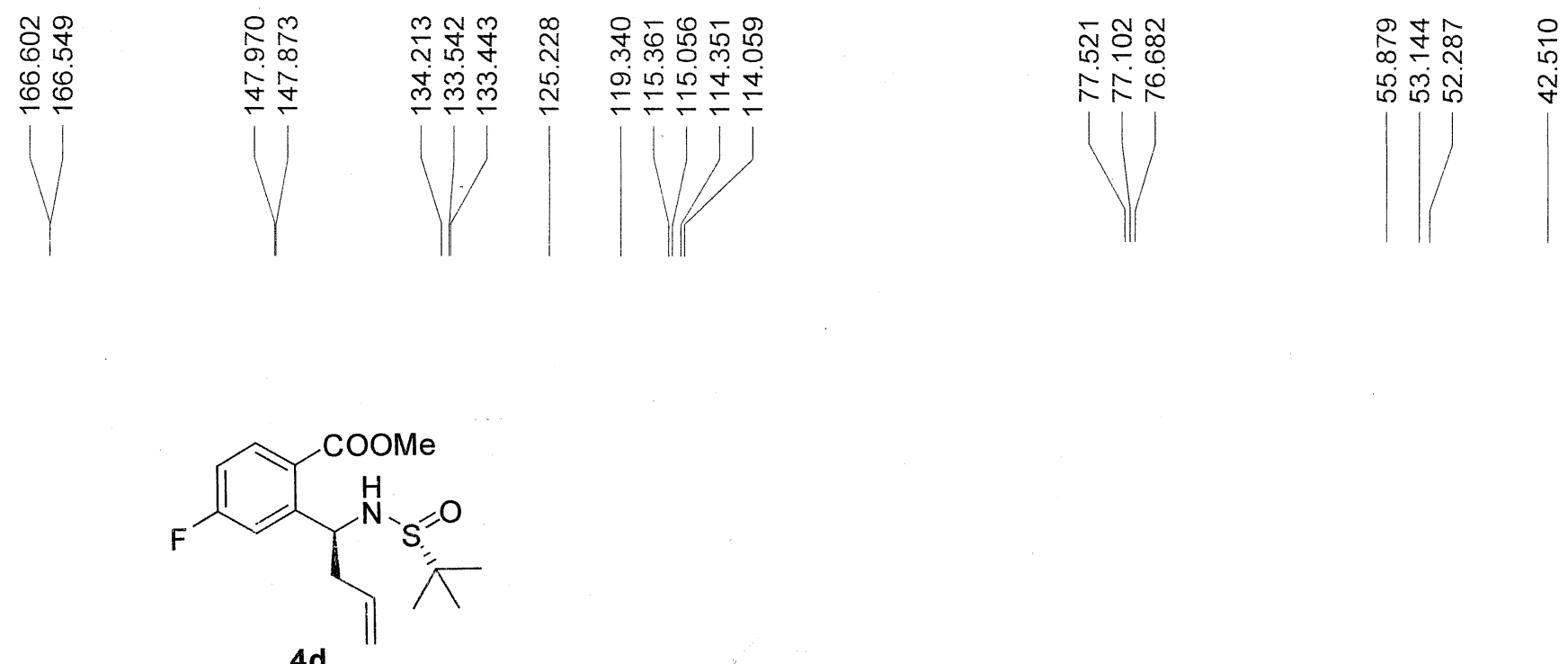

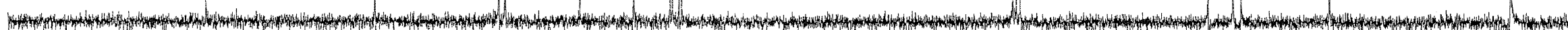




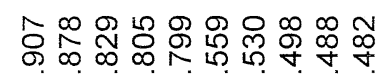

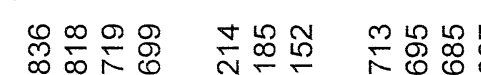

ம்

$\begin{array}{ll}\bar{\infty} & \bar{\delta} \\ 0 & \infty \\ \dot{\forall} & \infty\end{array}$

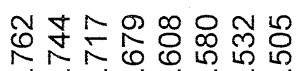

(1)

N N N N N N N

$1+111111$

I) .
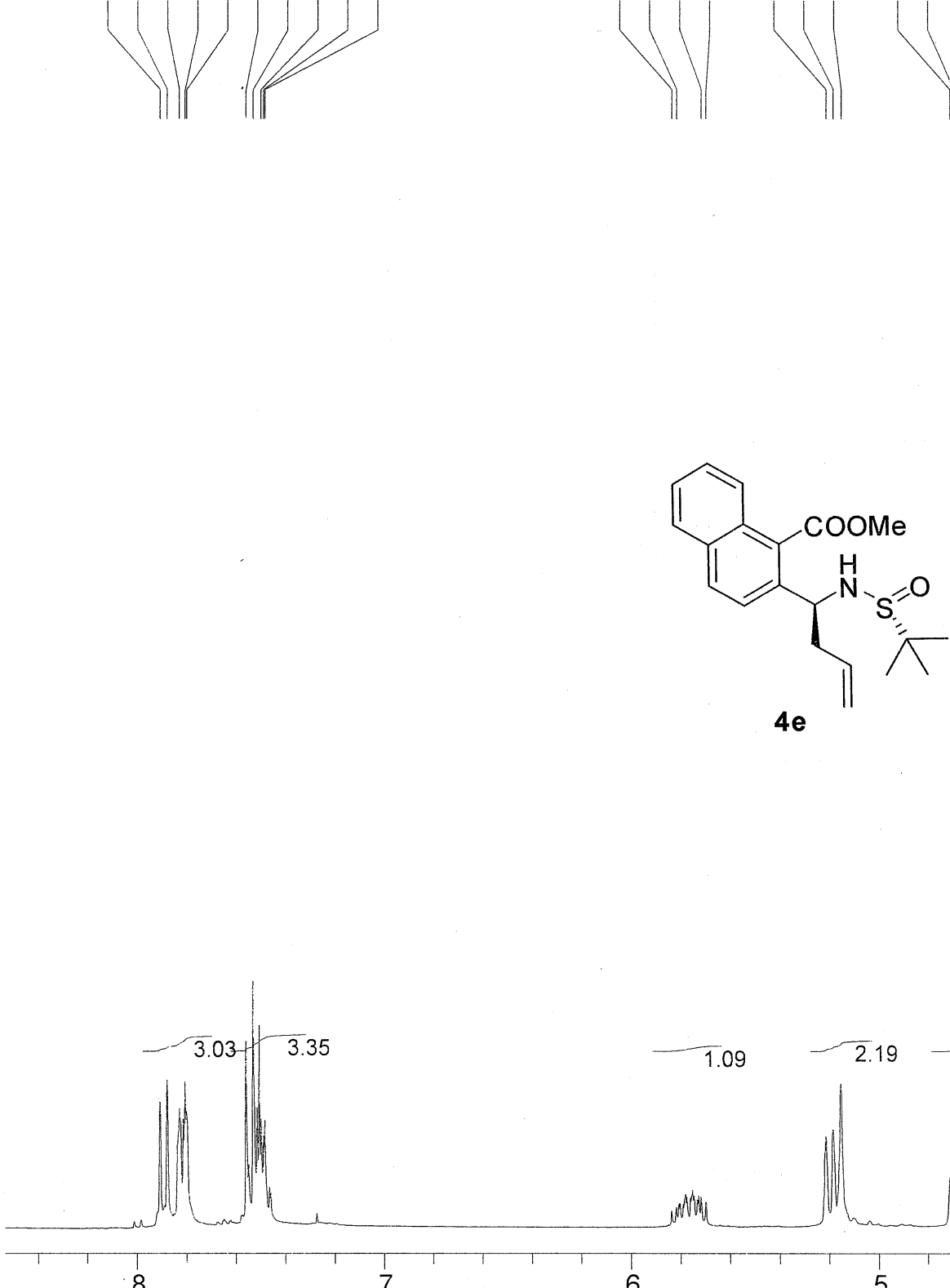

$\overline{1.09}$

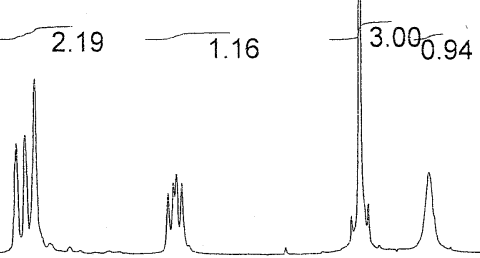

Min

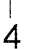



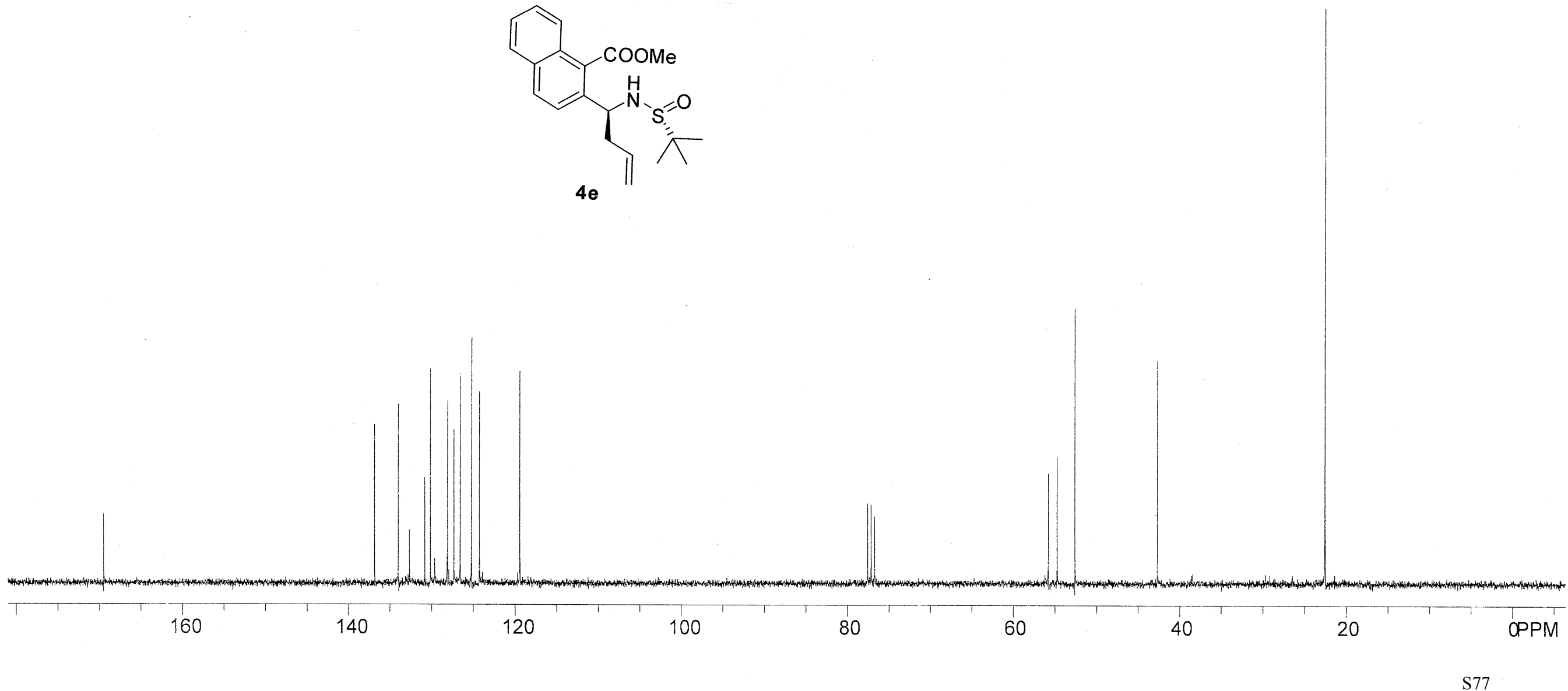


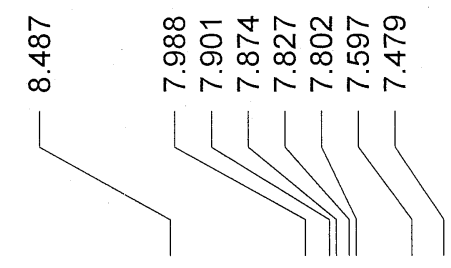

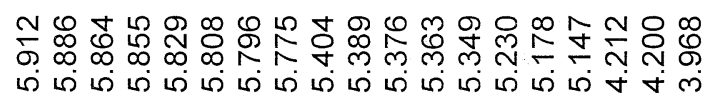

$\infty$

ก Ni Ni Ni i ivi

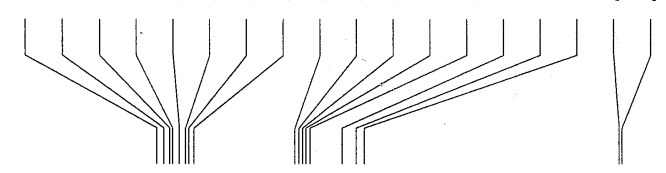

ก N N N

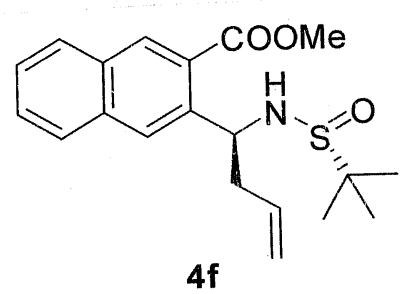

$4 f$

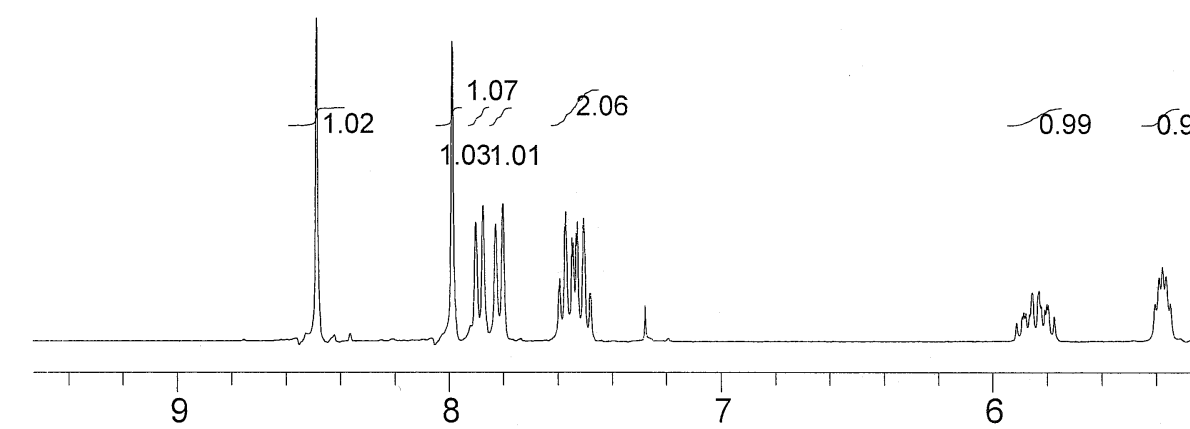

2

PPM 


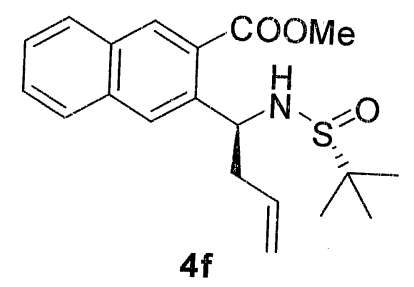




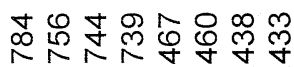

公公公公

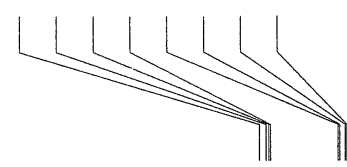

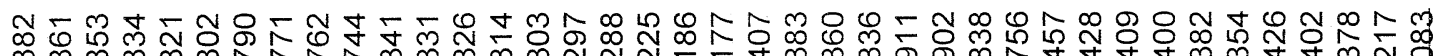

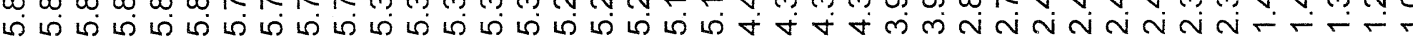

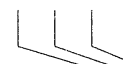

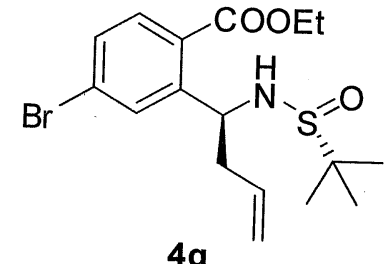

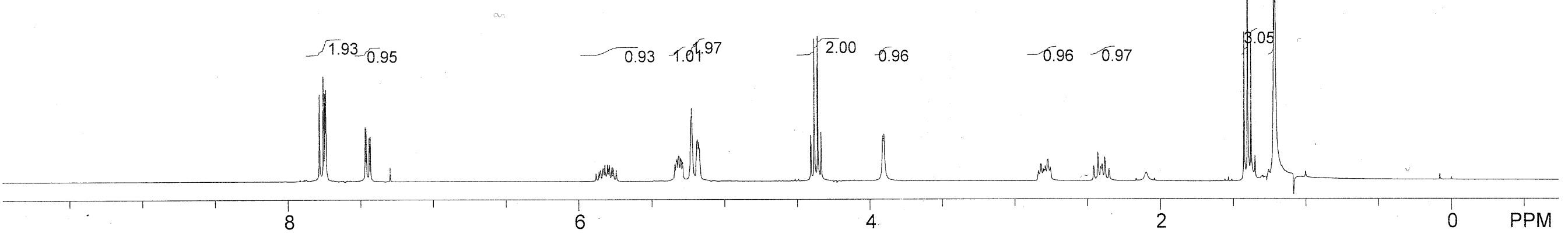




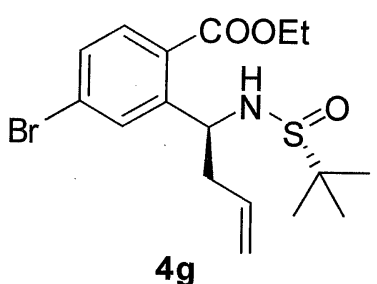

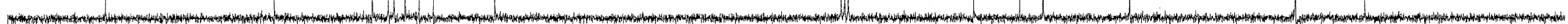




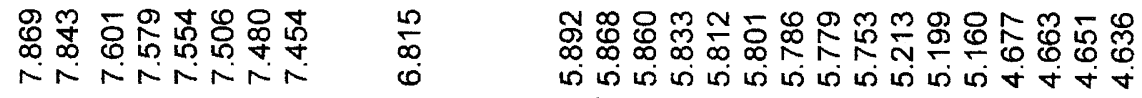

$1+1|1|$

in

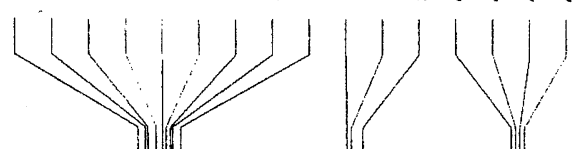

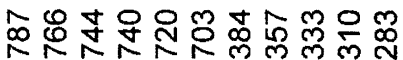

sor

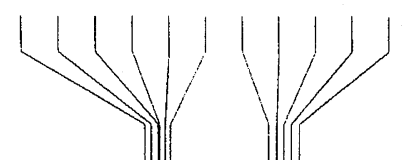

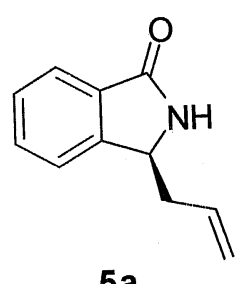

$5 a$

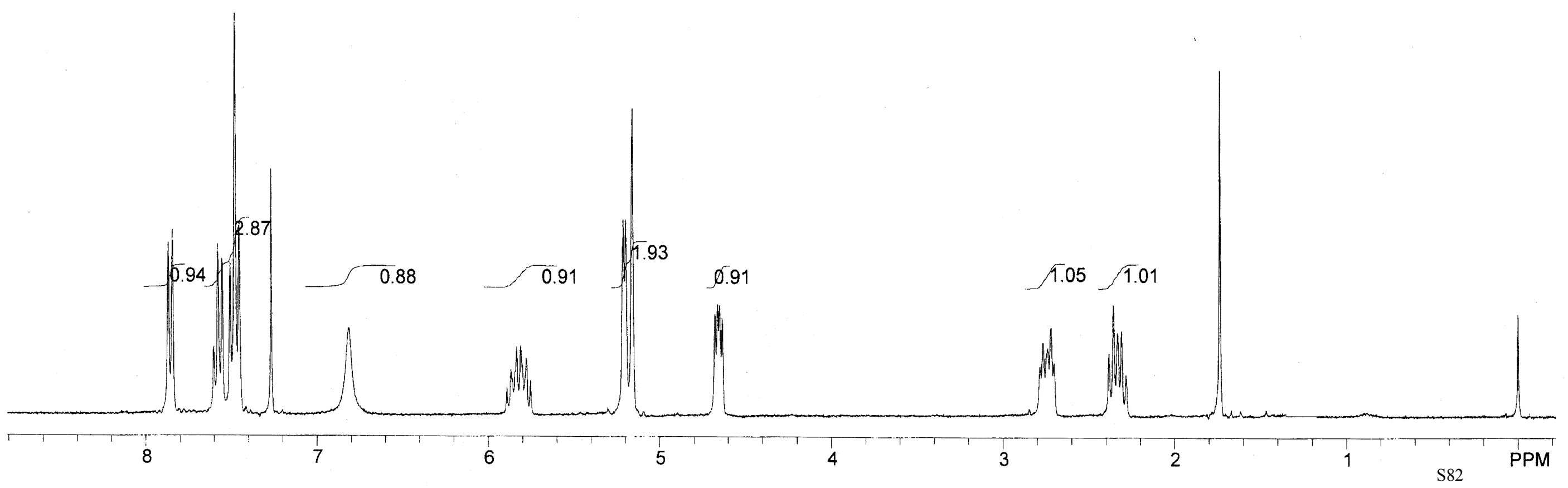




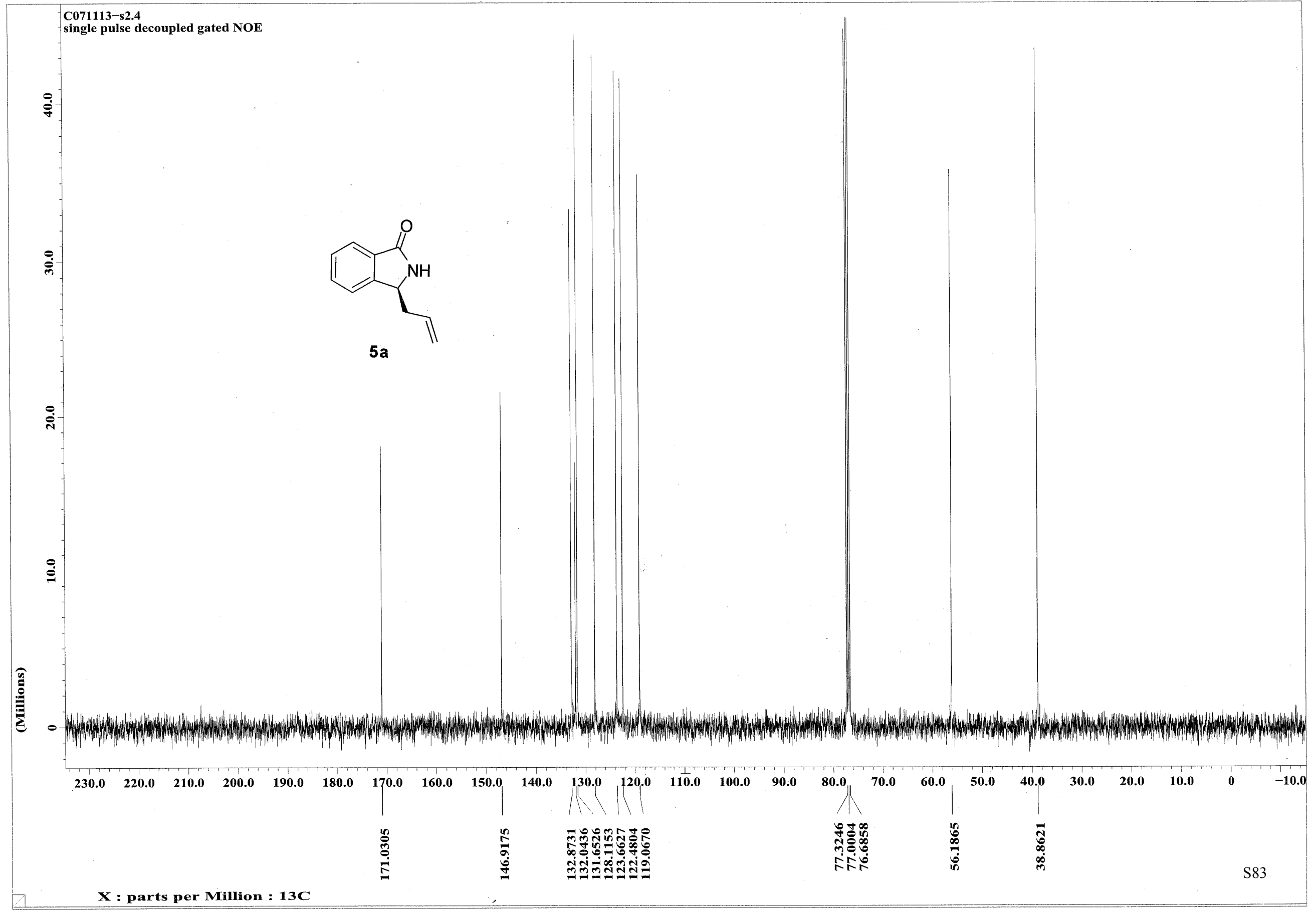




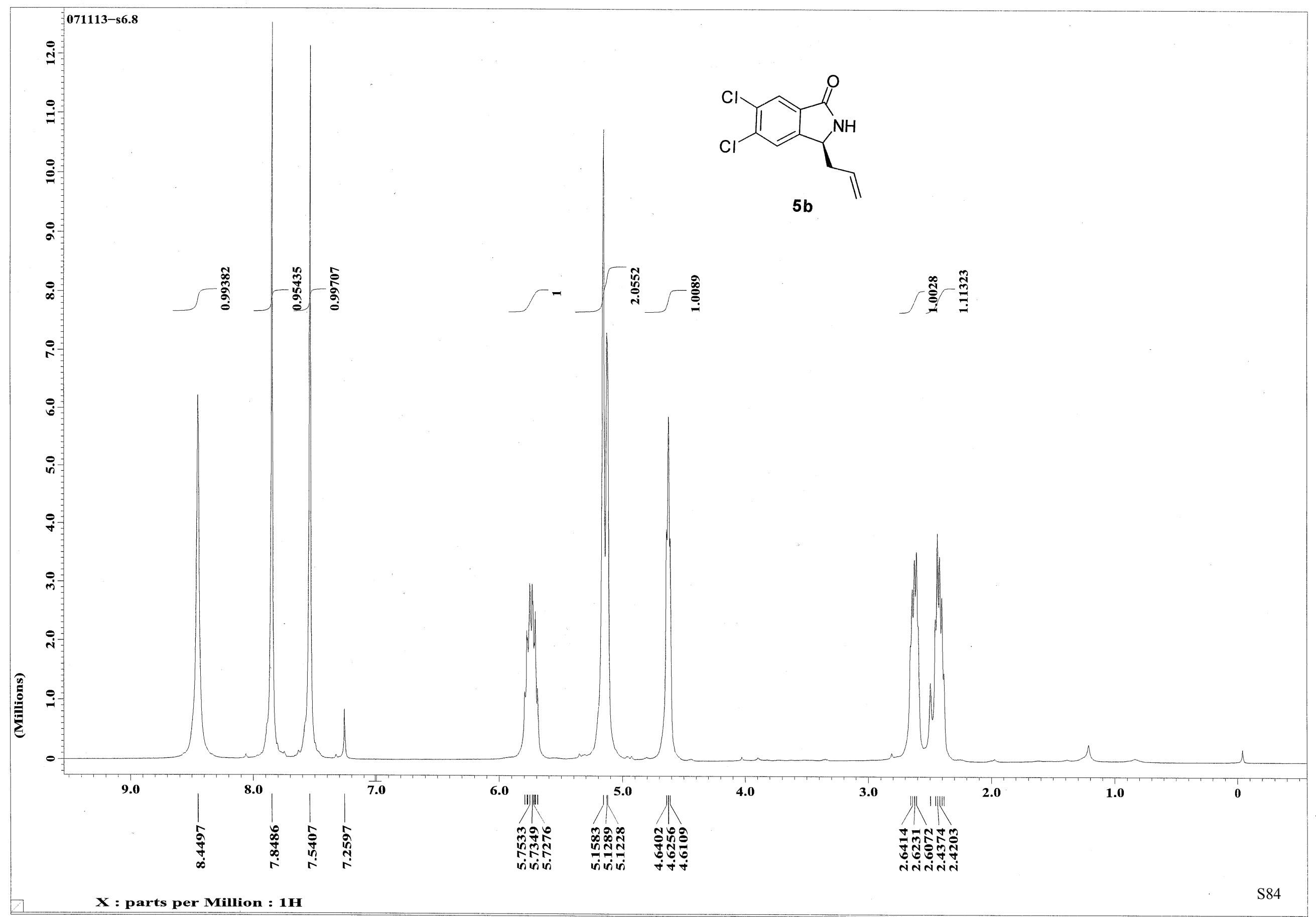




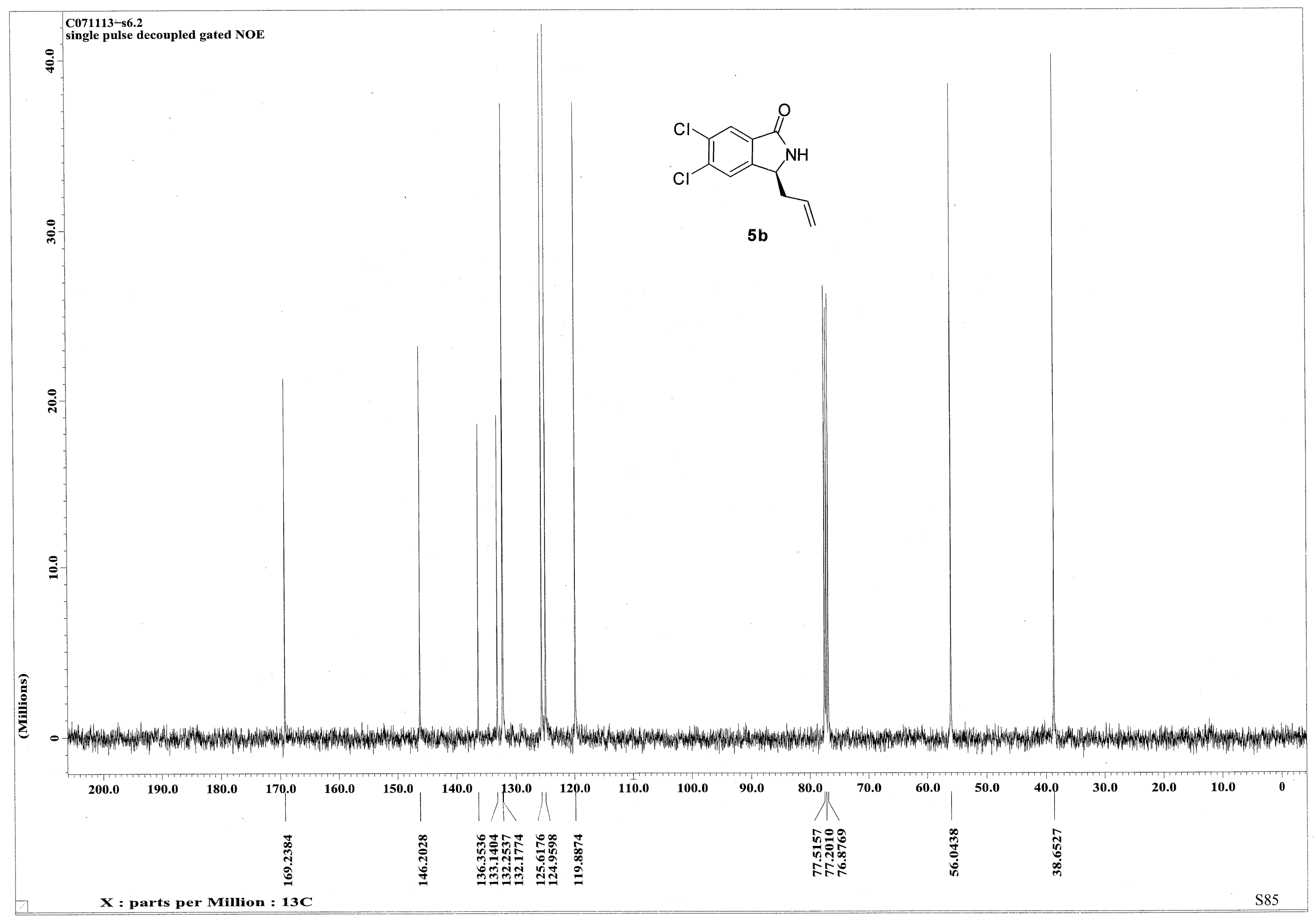




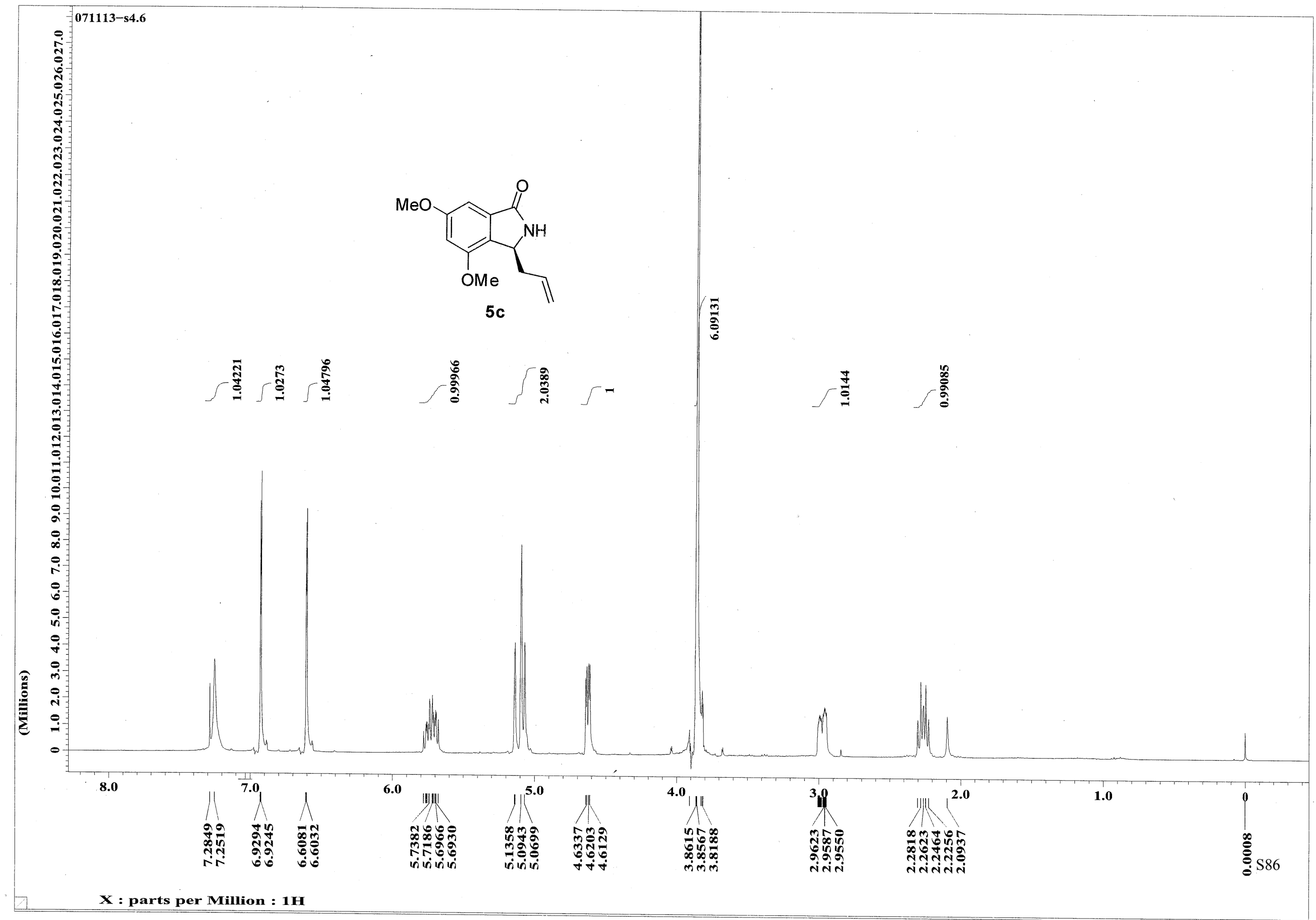




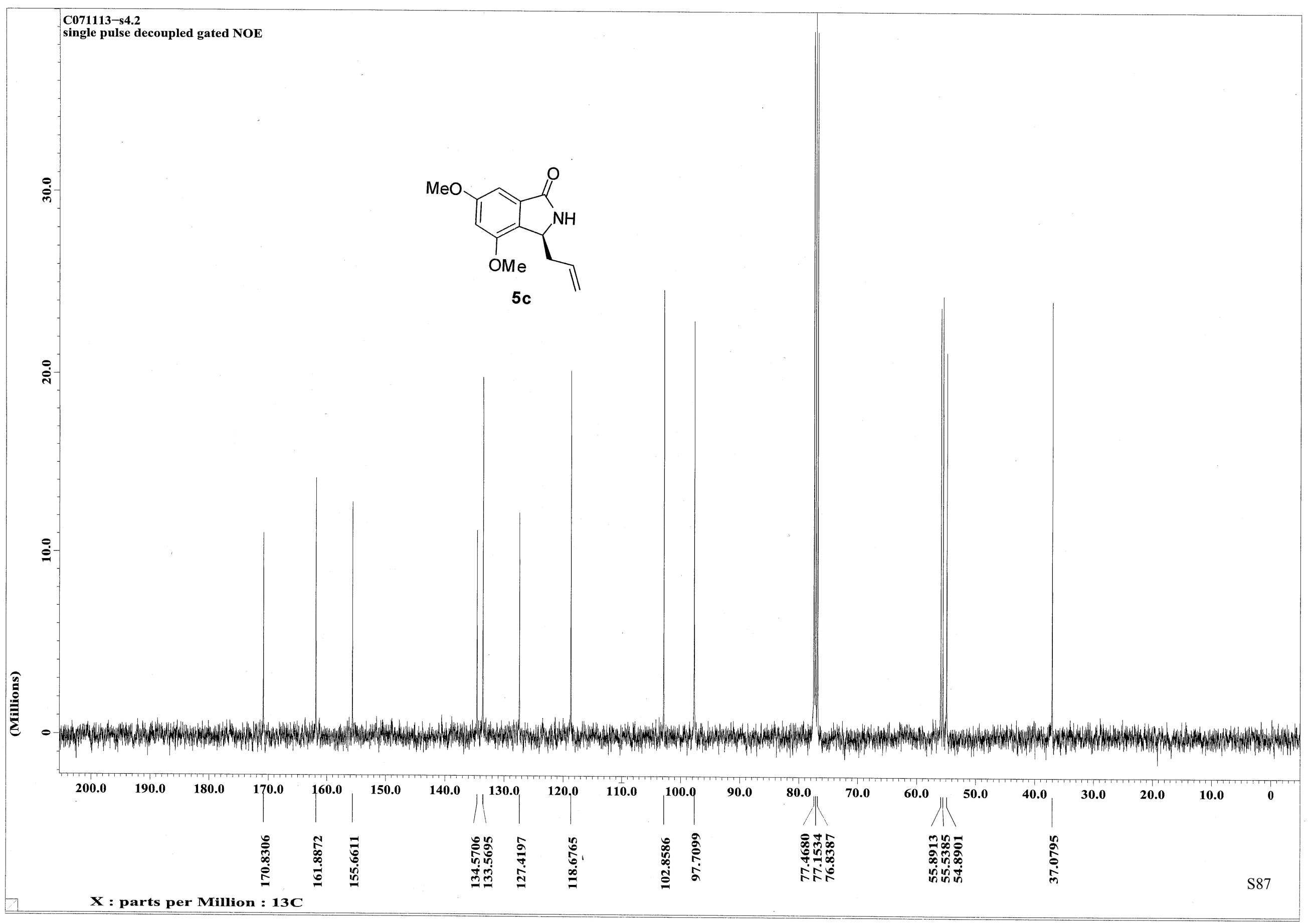




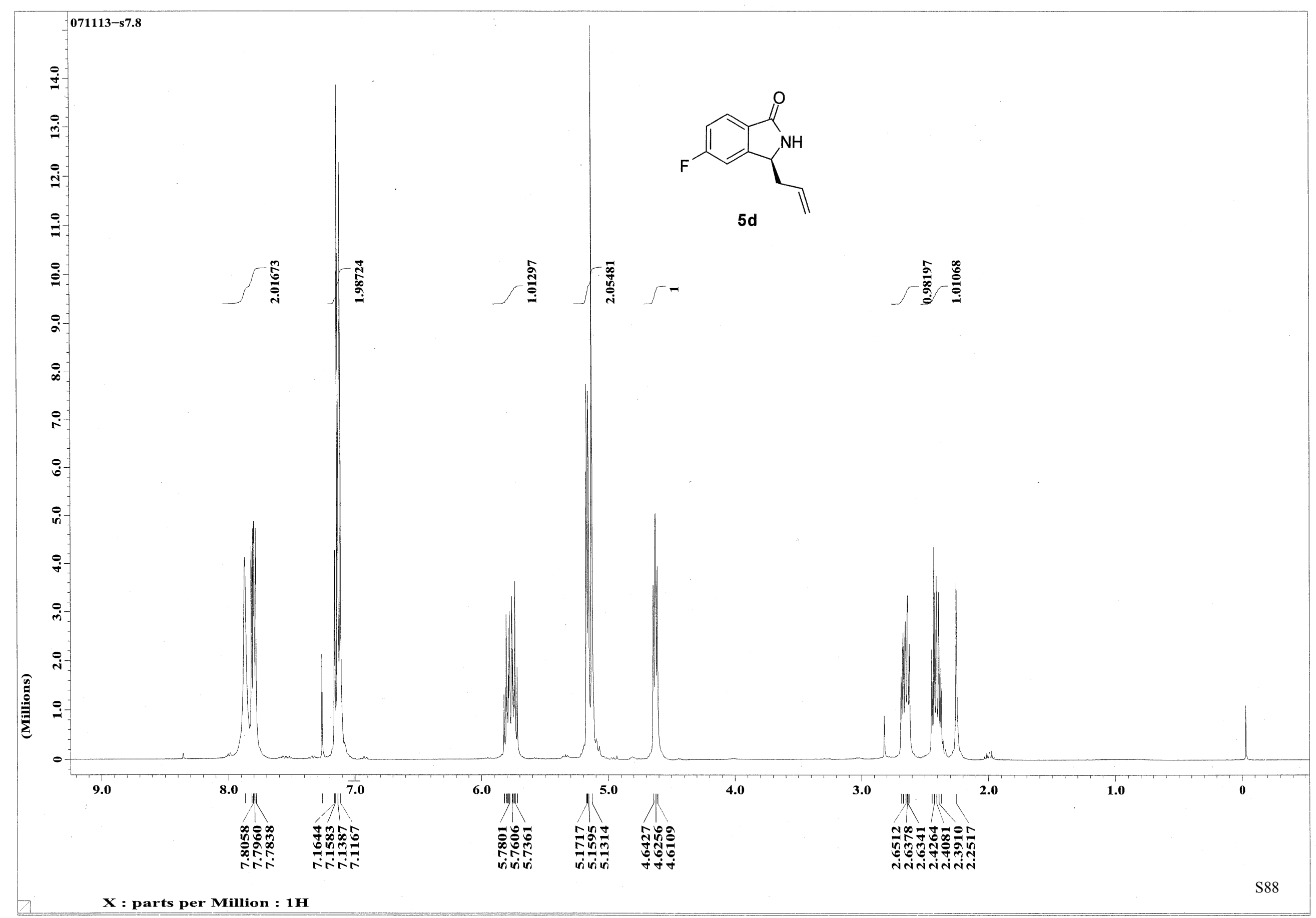




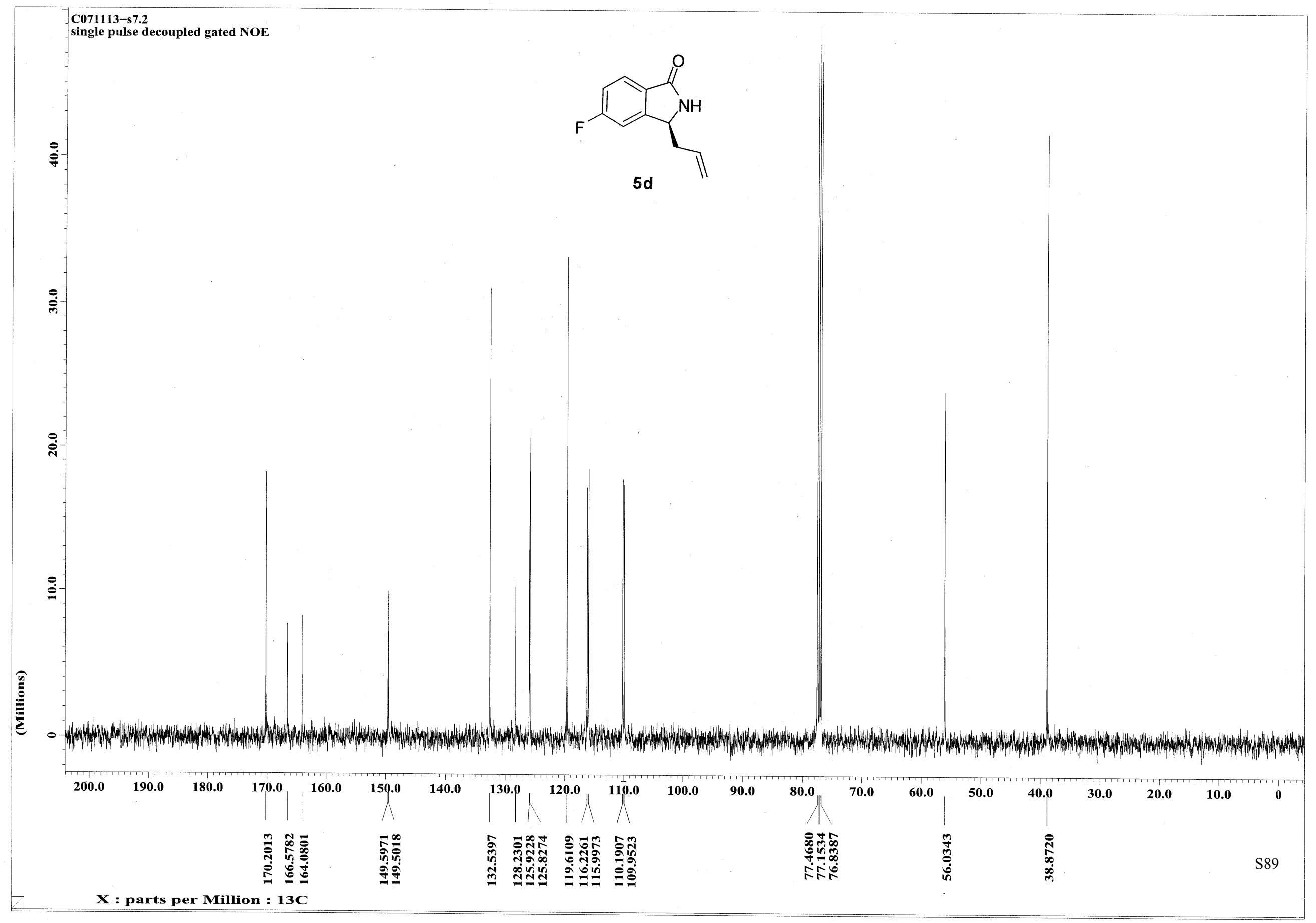




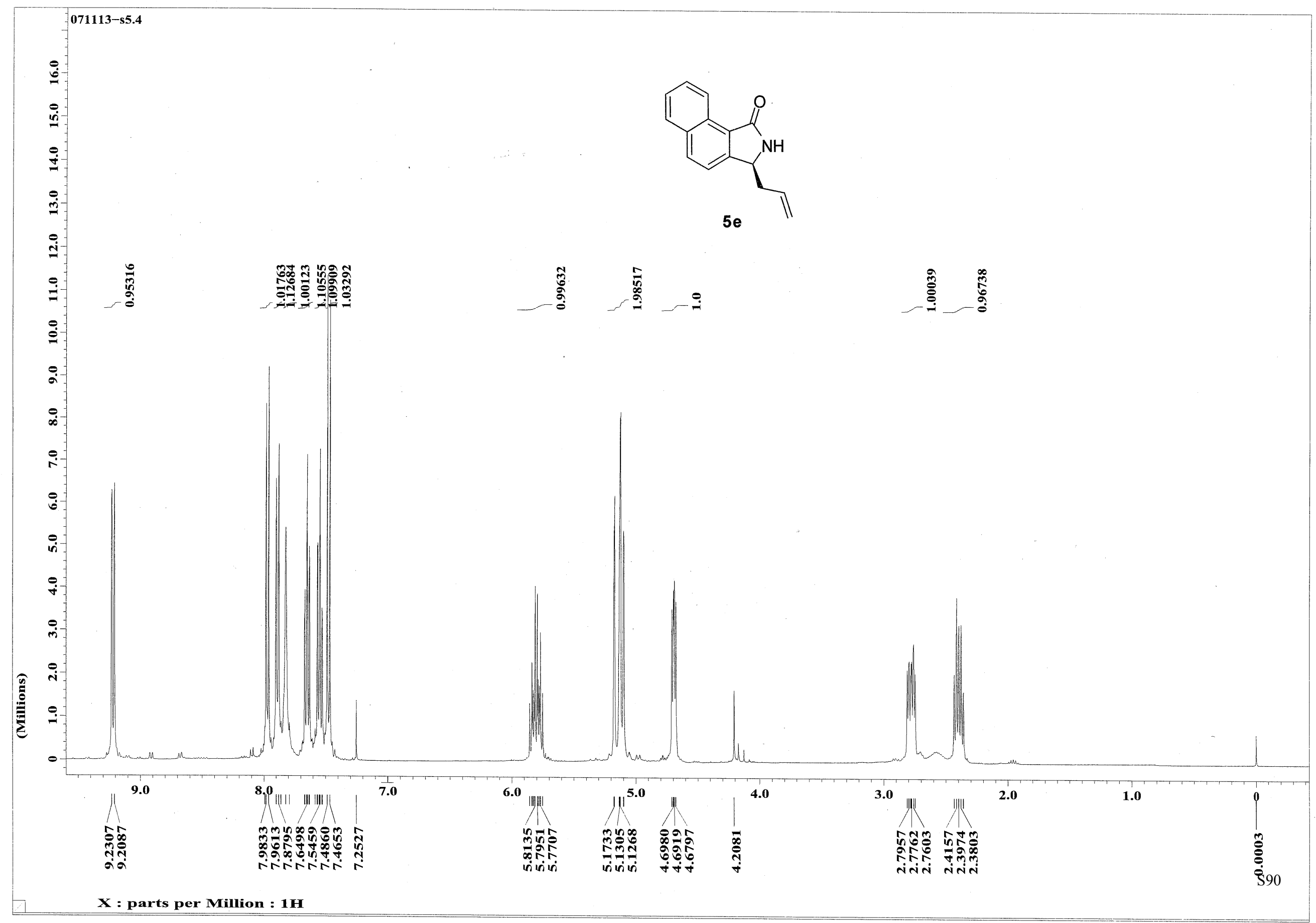




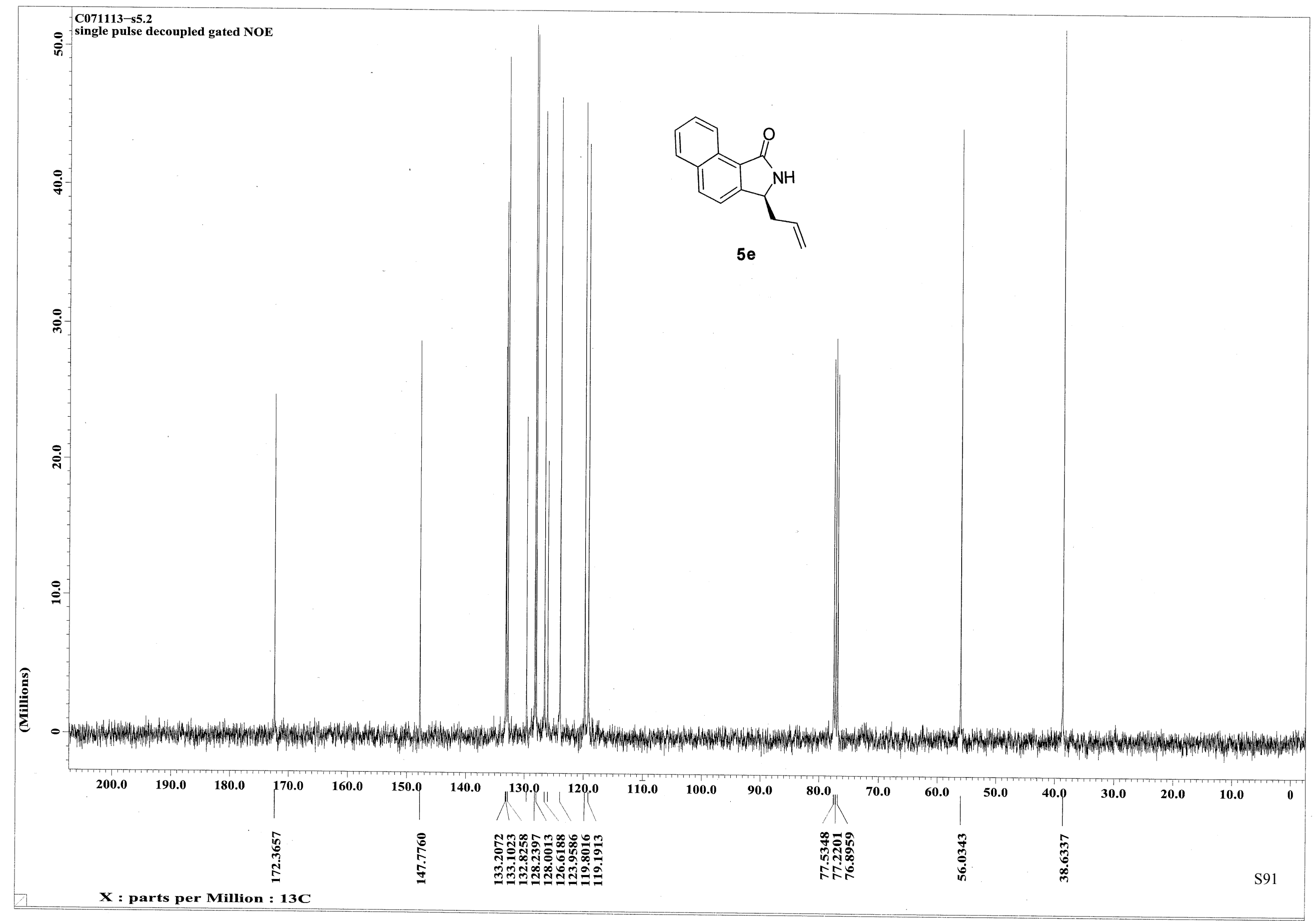




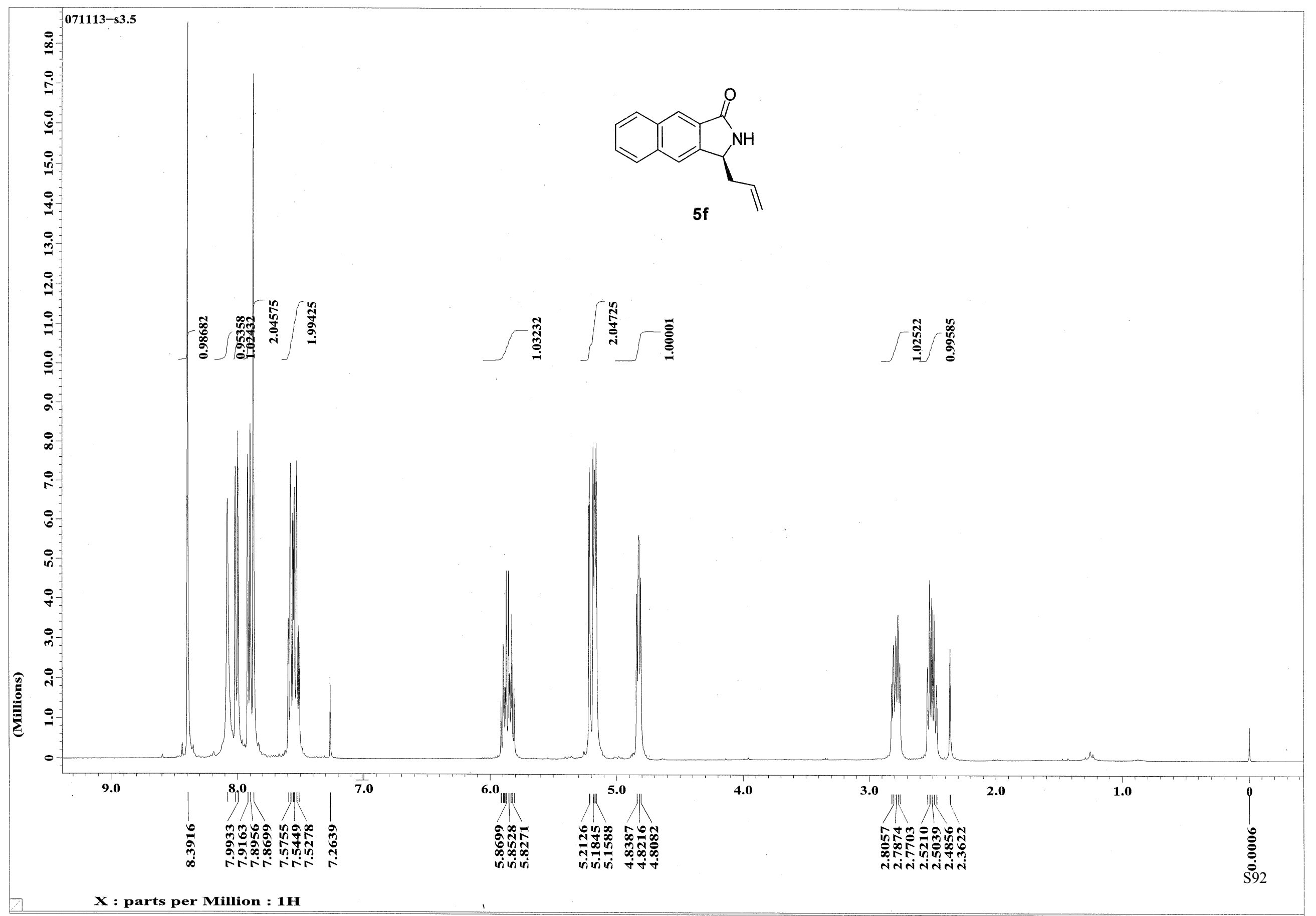




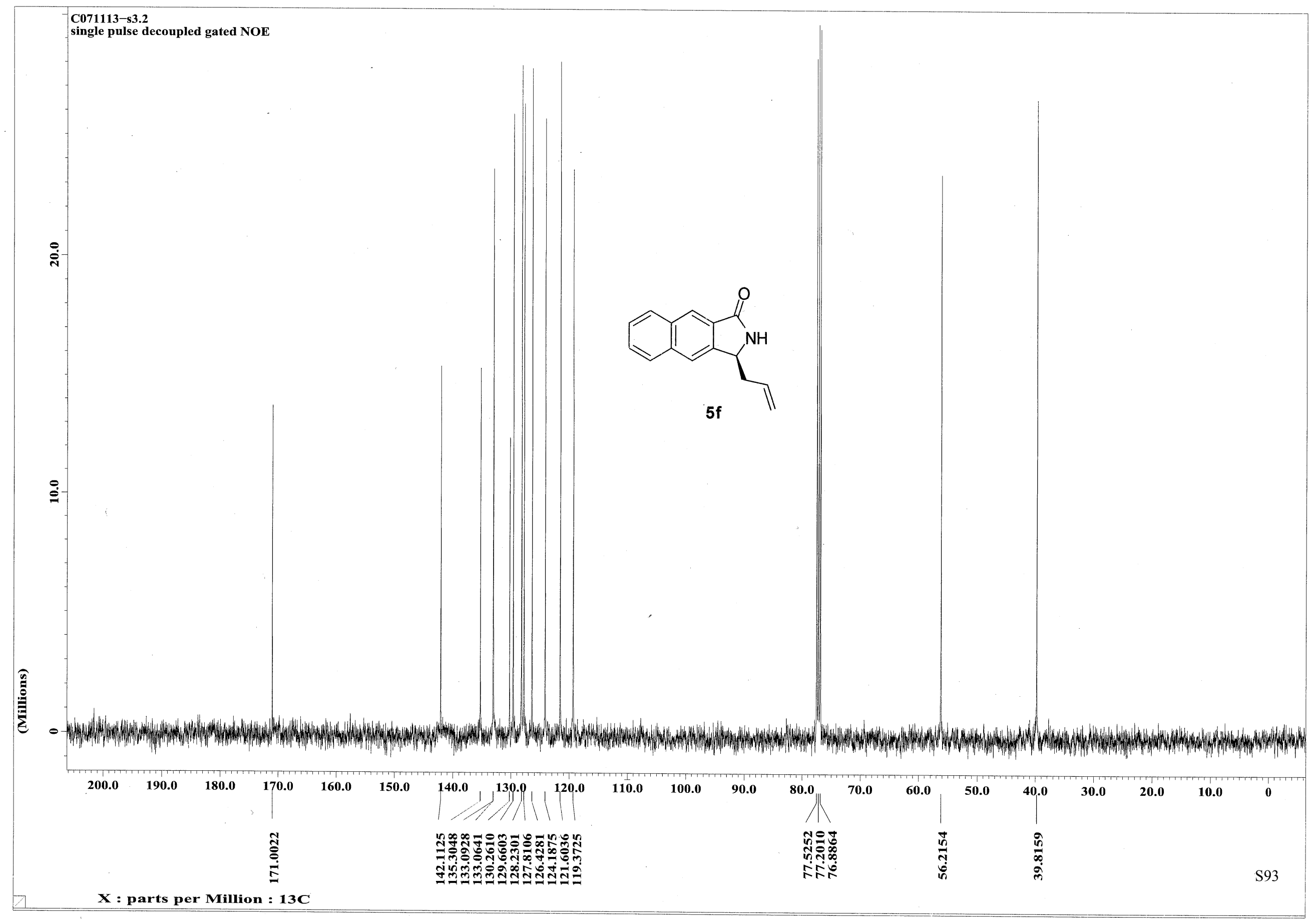




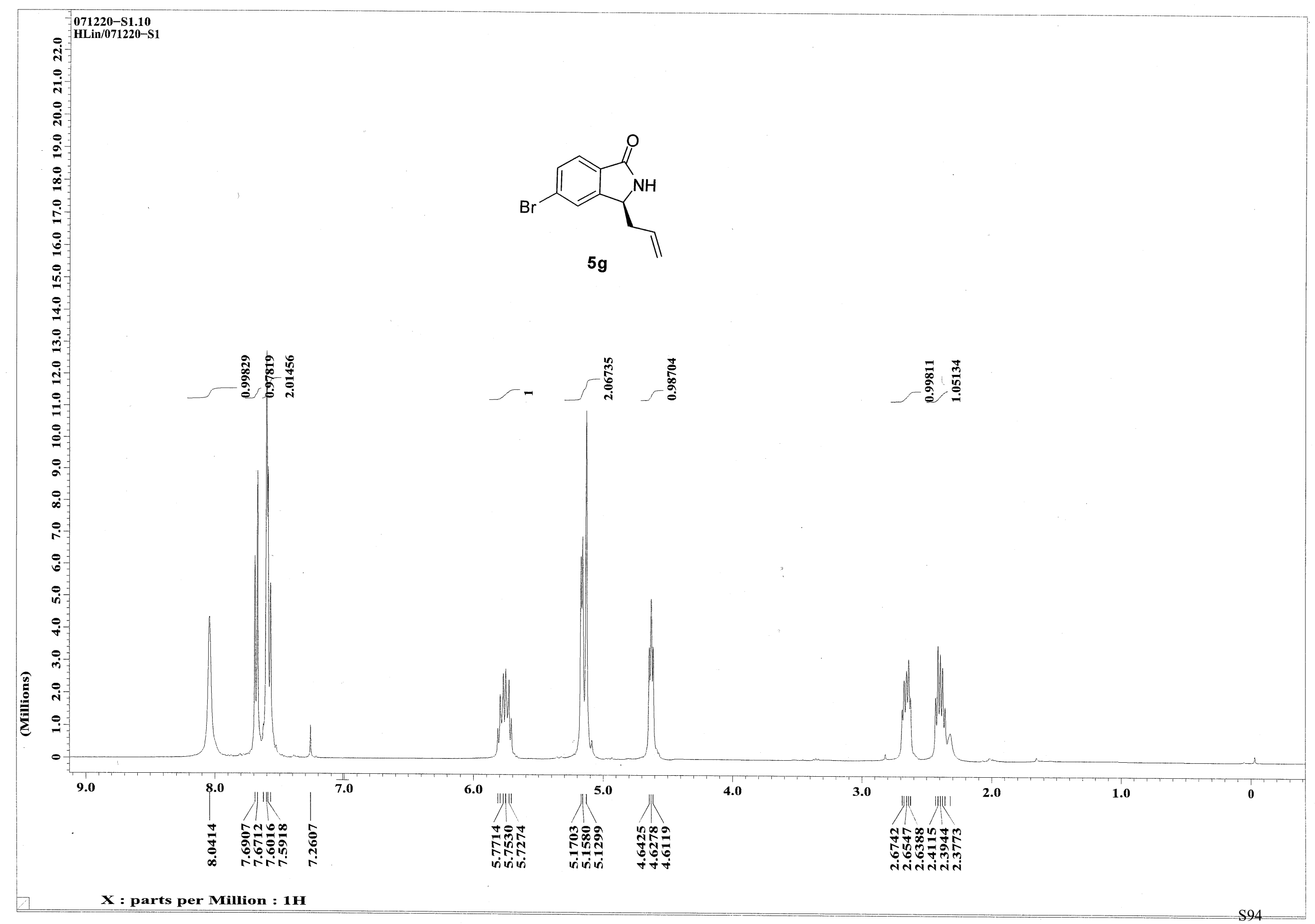




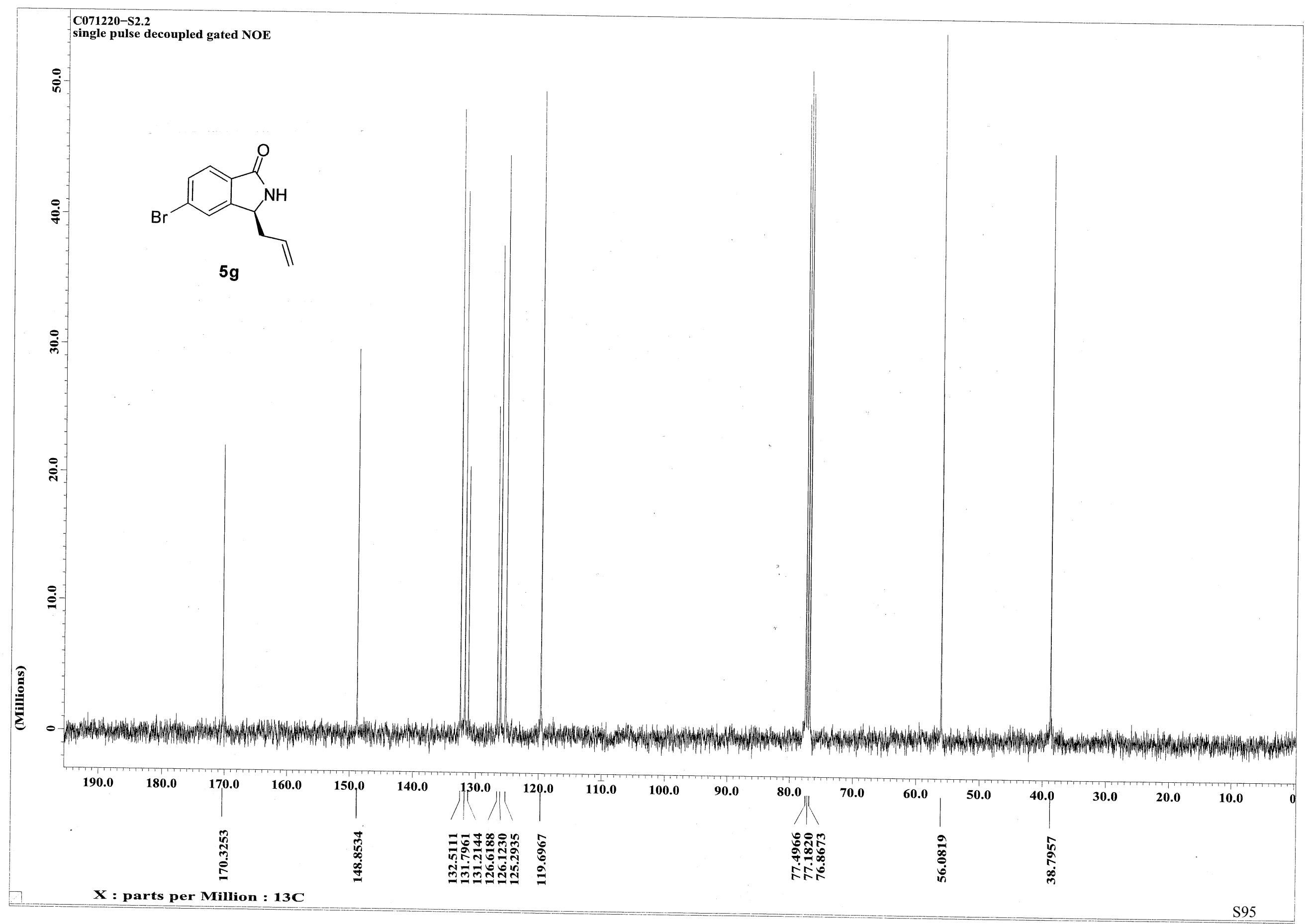

